\title{
Distributed Multiple Access for the Uplink of Multi-cell OFDMA Networks
}

\author{
by \\ Jing Dang \\ A Thesis submitted to \\ the Faculty of Graduate Studies and Research \\ in partial fulfilment of \\ the requirements for the degree of \\ Master of Applied Science \\ in \\ Electrical and Computer Engineering
}

Ottawa-Carleton Institute for Electrical and Computer Engineering

Department of Systems and Computer Engineering

Carleton University

Ottawa, Ontario, Canada

(C) 2013

Jing Dang 
The undersigned hereby recommend to the

Faculty of Graduate Studies and Research

acceptance of the thesis

\title{
Distributed Multiple Access for the Uplink of Multi-cell OFDMA Networks
}

\author{
Submitted by Jing Dang \\ in partial fulfilment of the requirements for the degree of \\ Master of Applied Science in Electrical and Computer Engineering
}

Professor Halim Yanikomeroglu, Thesis Supervisor

Professor Roshdy Hafez, Chair

Department of Systems and Computer Engineering
Ottawa-Carleton Institute for Electrical and Computer Engineering Department of Systems and Computer Engineering
Carleton University




\section{Abstract}

With the global deployment of the $4 \mathrm{G}$ (4th generation) wireless networks, it is expected that in the near future wireless data services will account for most of the traffic in the cellular networks. These data services may be characterized by highly diversified QoS (Quality of Service) requirements such as delay, data rate, and reliability. It may not be a good approach to use the current BS (Base Station)-centric scheduling as the universal scheduling method for all kinds of data services. Besides, centralized scheduling will require a substantial amount of overhead when the number of wireless devices is very large, due to the fact that in centralized scheduling the BSs need to collect the CSI (Channel State Information) of these devices to make appropriate decisions. This may be very challenging at BSs especially when the channel coherence time is low. Therefore, in this thesis we explore a distributed scheduling method for the next-generation cellular networks, in which the scheduling decisions are performed jointly by the wireless devices and the BSs in order to reduce the complexity at the BSs and to reduce the protocol overhead in terms of the bandwidth dedicated for the control signalling.

We propose a distributed multi-channel multiple access protocol for the uplink data transfer in TDD (Time-Division Duplex) mode for a multi-cell network. The protocol may be used as a low-overhead scheduling solution for delay-tolerant services especially with large numbers of devices, and can coexist with the legacy centralized scheduling schemes. In the proposed protocol, wireless devices play a key part by 
estimating channels, calculating certain indices, and (when certain criteria are met) sending channel reservation requests to BSs with the calculated indices for arbitration on channel allocation. BSs only need to do arbitration and to broadcast the necessary parameters of the protocol to guide the devices on their calculations, with no need of collecting devices' CSI or other channel quality parameters.

We devise two variants of the protocol for a) maximizing sum throughput, and b) maintaining proportional fairness. Both variants rely on the appropriate choices of the parameters of some decision making criteria for scheduling both at the devices and at the BSs. For the sum throughput maximization, we prove that if the number of devices is large enough, the throughput of the proposed protocol approaches to that of the centralized scheduling. We conduct simulations to examine the performance analysis. For the sum throughput maximization, the simulation results show that we can achieve this goal with a low level of control overhead. For proportional fairness approximation, we can appropriately select the window size for the average rate calculation to trade-off between fairness and throughput; furthermore, by setting proper thresholds we can also achieve this trade-off with a relatively low level of control overhead. Finally, we discuss some implementation issues such as channel request collision avoidance and the downlink data transfer. 


\section{Acknowledgments}

First, I would like to express my sincere appreciation and respect for my supervisor Prof. Halim Yanikomeroglu for his generous efforts in guiding this thesis research. Without his valuable supervision, this work would not have been possible. All the results of this thesis came from many face to face discussions with Prof. Halim Yanikomeroglu. During this research period, I was also impressed by his expertise in wireless communications. He taught me not only the broad range of wireless communications but also the details and latest advancements of many research directions. He also showed to me the management skills of relating academic research work to industrial requirements. I am very lucky to have such an excellent professor and a fruitful research group in my thesis research. I also want to express gratitude to the valuable help and suggestions from all the members of our research group.

I would also like to thank Huawei Canada and the Ontario Ministry of Economic Development and Innovation's ORF-RE (Ontario Research Fund - Research Excellence) program for funding the research presented in this thesis.

Last but not the least, I would like to express my special thanks and respect to my family members. Thanks for the effort and time they spent with me. 


\section{Table of Contents}

$\begin{array}{lll}\text { Abstract } & \text { iii }\end{array}$

$\begin{array}{lll}\text { Acknowledgments } & \text { v }\end{array}$

Table of Contents vi

List of Tables $\quad$ ix

List of Figures $\quad$ x

List of Acronyms xii

$\begin{array}{ll}\text { List of Symbols } & \text { Xv }\end{array}$

1 Introduction 1

1.1 Thesis Problem Statement and Motivation . . . . . . . . . . . . . 1

1.2 Thesis Contributions . . . . . . . . . . . . . . . . 2

1.3 Thesis Organization . . . . . . . . . . . . . . . . . . 3

2 Overview of Scheduling in Wireless Networks 4

2.1 A Brief Introduction of Medium Access Control Protocols . . . . . . . 4

2.1.1 Problems and Challenges in Wireless Medium Access Control 6

2.1.2 Classification of Wireless MAC Protocols . . . . . . . . . . . 10

2.1.3 Performance Metrics of Medium Access Control Protocols . . 12 
2.2 Discussion on Centralized Scheduling and Challenges . . . . . . . . . 14

2.3 Distributed Scheduling/Random Access Discussion and Literature Review .................................... 14

2.4 Summary and Research Proposals . . . . . . . . . . . . . . . . . . . 18

3 System Model Description $\quad 21$

3.1 Introduction . . . . . . . . . . . . . . . . . . . 21

3.2 Distributed Scheduling Framework . . . . . . . . . . . . . . . . 22

3.3 Proposed Distributed Scheduling Algorithms . . . . . . . . . . . . . 25

3.3.1 Algorithm for Scenario I: Sum Throughput Maximization . . . 25

3.3.2 Algorithm for Scenario II: Proportional Fairness Approximation 28

3.4 Summary . . . . . . . . . . . . . . . . . . . 32

4 Mathematical Analysis $\quad 33$

4.1 Homogeneous Channel Model . . . . . . . . . . . . . . . . . . . . . . 33

4.1.1 Power Allocation with Channel Inversion . . . . . . . . . . . . 39

4.1.2 Power Allocation with Interference Boundary . . . . . . . . . 45

4.1.3 Power Allocation With Equality . . . . . . . . . . . . 47

4.2 Heterogeneous Channel Model . . . . . . . . . . . . . . . . . . . . . . 48

4.2.1 Power Allocation with Channel Inversion . . . . . . . . . . . 51

4.2.2 Power Allocation with Equality . . . . . . . . . . . . 53

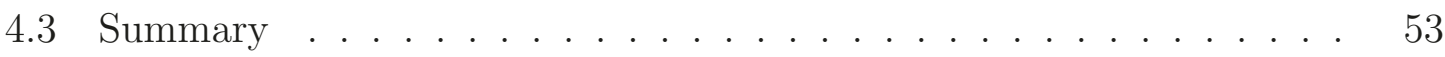

5 Simulation and Numerical Results $\quad 54$

5.1 Simulation Platform Setup . . . . . . . . . . . . . . . 54

5.2 Simulation Results for Scenario I: Sum Throughput Maximization . . 55

5.2.1 Sum Throughput Maximization . . . . . . . . . . 55

5.2 .2 The Number of Candidate Users . . . . . . . . . . . . . . . 62 
5.2.3 Spectral Efficiency with Different Power Allocation . . . . . . 65

5.2 .4 Fairness Issue in Scenario I . . . . . . . . . . . . . . . 67

5.2.5 Mass Traffic Handling in Scenario I . . . . . . . . . . . . . . 70

5.3 Simulation Results for Scenario II: Proportional Fairness Approximation 71

5.3.1 Distribution of the Selected Criterion Values with Different Window Sizes . . . . . . . . . . . . . . . 73

5.3.2 Cumulative Distribution Function of User Data Rate . . . . . 85

5.3.3 Steps for the Selection of Window Sizes and Thresholds . . . . 87

5.3.4 Mass Traffic Handling in Scenario II . . . . . . . . . . . . 87

5.4 Comparisons on the Results of Two Scenarios . . . . . . . . . . . . 88

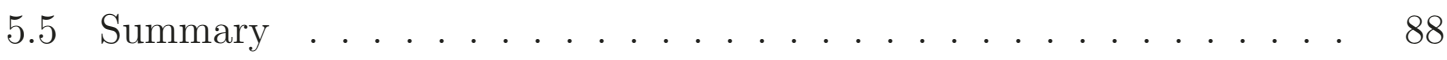

6 Discussion on Some Implementation Issues $\quad 89$

6.1 Channel Request Collision Avoidance . . . . . . . . . . . . . . . . 89

6.2 Downlink Data Transfer Discussion . . . . . . . . . . . . . . . 91

6.3 Pilot Signal and System Information Broadcast . . . . . . . . . . . . 92

6.4 Dynamic Network Adaption . . . . . . . . . . . . . . . . . 92

6.5 Quantization of Criterion Values . . . . . . . . . . . . . . 92

7 Conclusions and Future Research Directions 93

7.1 Conclusions . . . . . . . . . . . . . . . . . . . . . 93

7.2 Future Research Directions _. . . . . . . . . . . . . . 95

7.2.1 Power Allocation . . . . . . . . . . . . . . . . . 95

7.2 .2 Load Balancing . . . . . . . . . . . . . . . 95

7.2 .3 QoS Provisioning . . . . . . . . . . . . . 95

$\begin{array}{lc}\text { List of References } & 96\end{array}$

viii 


\section{List of Tables}

2.1 Summary of literature review. . . . . . . . . . . . . . . . . . . . 19

2.2 Comparison of our work with literature. . . . . . . . . . . . . . 20

4.1 Summary of notations. . . . . . . . . . . . . . . . 34

5.1 Summary of simulation parameters for Scenario I. . . . . . . . . . . . 55

5.2 Summary of simulation parameters for Scenario II. . . . . . . . . . . 72

5.3 Summary of suitable thresholds for different window sizes. . . . . . . 84 


\section{List of Figures}

2.1 Hidden terminal problem and exposed terminal problem [1]. . . . . . 8

2.2 Classification of wireless MAC protocols. . . . . . . . . . . . . . . 10

3.1 System model. . . . . . . . . . . . . . . . . . . . . . 21

3.2 Distributed scheduling framework flow chart . . . . . . . . . . . . 23

3.3 A possible implementation block diagram. . . . . . . . . . . . . . 24

5.1 A multi-cell network with four BSs. ${ }^{1} \ldots \ldots \ldots$. . . . . . . 54

5.2 Average spectral efficiency per BS over all RBs $(\alpha=0 \mathrm{~dB}) \ldots \ldots . . \quad 57$

5.3 Average spectral efficiency per BS over all RBs $(\alpha=-10 \mathrm{~dB}) . \ldots . \quad 57$

5.4 Average spectral efficiency per BS over all RBs $(\alpha=-20 \mathrm{~dB})$. . . . . 58

5.5 Average spectral efficiency per BS over all RBs $(\alpha=-30 \mathrm{~dB}) . \ldots 58$

5.6 Average spectral efficiency per BS over all RBs $(\alpha=-40 \mathrm{~dB})$. . . . . 59

5.7 Average spectral efficiency per BS over all RBs $(\alpha=-50 \mathrm{~dB})$. . . . 59

5.8 Normalized no. of candidate users per RB $(\alpha=0 \mathrm{~dB}) \ldots \ldots . . . . .662$

5.9 Normalized no. of candidate users per RB $(\alpha=-10 \mathrm{~dB}) \ldots \ldots 2$

5.10 Normalized no. of candidate users per RB $(\alpha=-20 \mathrm{~dB}) \ldots \ldots$

5.11 Normalized no. of candidate users per RB $(\alpha=-30 \mathrm{~dB}) \ldots \ldots 3$

5.12 Normalized no. of candidate users per RB $(\alpha=-40 \mathrm{~dB}) . \ldots . . . .64$

5.13 Normalized no. of candidate users per RB $(\alpha=-50 \mathrm{~dB}) . \ldots . . .64$

5.14 Average spectral efficiency per BS with different power allocations. . 66

5.15 Jain's fairness index for schemes DS, DS2, SH, CS. . . . . . . . . . 68 
5.16 CDF of average user data rate using DS scheme. . . . . . . . . . . . 69

5.17 CDF of average user data rate using DS2 scheme. . . . . . . . . . . . 69

5.18 CDF of average user data rate using SH scheme. . . . . . . . . . . . 70

5.19 CDF of average user data rate using CS scheme. . . . . . . . . . . . 70

5.20 Simulation with Jain's fairness index. . . . . . . . . . . . . . . . 73

5.21 Average selected criterion values $\mathrm{AV}\left(\mathrm{CV}^{*}\right)$ vs different window sizes. $\quad 74$

5.22 Histogram of the selected criterion values $\mathrm{CV}^{*}, \mathrm{WIN}=30 \ldots \ldots$

5.23 Histogram of the selected criterion values $\mathrm{CV}^{*}, \mathrm{WIN}=40 \ldots \ldots . . \quad 75$

5.24 Histogram of the selected criterion values $\mathrm{CV}^{*}, \mathrm{WIN}=50 \ldots \ldots 76$

5.25 Histogram of the selected criterion values $\mathrm{CV}^{*}, \mathrm{WIN}=60 \ldots \ldots 76$

5.26 Histogram of the selected criterion values $\mathrm{CV}^{*}, \mathrm{WIN}=70 \ldots \ldots 77$

5.27 Histogram of the selected criterion values $\mathrm{CV}^{*}, \mathrm{WIN}=80 \ldots \ldots 77$

5.28 Histogram of the selected criterion values $\mathrm{CV}^{*}, \mathrm{WIN}=90 \ldots \ldots$

5.29 Histogram of the selected criterion values $\mathrm{CV}^{*}, \mathrm{WIN}=100 \ldots . . .78$

5.30 CDF of the selected criterion values $\mathrm{CV}^{*}$. . . . . . . . . . . . 79

5.31 Histogram of no. of candidate users at different thresholds (WIN=30). 80

5.32 Histogram of no. of candidate users at different thresholds (WIN=40). 80

5.33 Histogram of no. of candidate users at different thresholds (WIN=50). 81

5.34 Histogram of no. of candidate users at different thresholds (WIN=60). 81

5.35 Histogram of no. of candidate users at different thresholds (WIN=70). 82

5.36 Histogram of no. of candidate users at different thresholds (WIN=80). 82

5.37 Histogram of no. of candidate users at different thresholds (WIN=90). 83

5.38 Histogram of no. of candidate users at different thresholds (WIN=100). 83

$5.39 \mathrm{CDF}$ of user data rate at different time periods. . . . . . . . . . . 86

6.1 Channel request collision avoidance. . . . . . . . . . . . . . . . . . 90 


\section{List of Acronyms}

Acronyms Definition

\begin{tabular}{ll}
\hline \hline AWGN & Additive White Gaussian Noise \\
bps & Bits per second \\
BS & Base Station \\
CAD-MAC & Channel-Aware Distributed Medium Access Control \\
CAMCRA & Channel Aware Multi-Carrier Random Access scheme \\
CDF & Cumulative Distribution Function \\
CDMA & Code-Division Multiple Access \\
CDMA2000 & A CDMA standard \\
CRA & Contention Resolution Algorithm \\
CS & Centralized Scheduling \\
CSI & Channel State Information \\
CSMA & Decibels
\end{tabular}


DL

Downlink

DS

Distributed Scheduling

DOMRA Decentralized Optimization for Multichannel Random Access

DOS Distributed Opportunistic Scheduling

EV-DO Evolution-Data Optimized

FDD Frequency-Division Duplex

FDMA Frequency-Division Multiple Access

GSM Global System for Mobile communications

HDR High Data Rate

LAN Local Area Networks

LTE Long Term Evolution

M2M Machine to Machine

MAC Medium Access Control

MIMO Multiple-Input Multiple-Output

OAM Operation and Management

OFDM Orthogonal Frequency-Division Multiplexing

OFDMA Orthogonal Frequency-Division Multiple Access

OMC-Aloha OFDM-based Multi-Channel Aloha 
Proportional Fairness

SINR Signal-to-Interference-plus-Noise Ratio

SNR Signal-to-Noise Ratio

TDD Time-Division Duplex

TDMA Time-Division Multiple Access

UL Uplink

WCDMA Wideband Code-Division Multiple Access

WLAN Wireless Local Area Networks

WPF Weighted Proportional Fairness 


\section{List of Symbols}

\section{Symbols Definition}

\begin{tabular}{ll}
\hline \hline$N$ & The number of BSs \\
$L$ & The number of channels in every BS \\
$M$ & The number of users \\
$H_{t h}$ & Threshold of channel power gain \\
$\alpha^{(k)}$ & Channel $k$ of BS $j$ \\
$h_{i j}^{(k)}$ & Channel power gain of user $i$ for channel $k$ of BS $j$ \\
$f(h)$ & Probability density function of channel power gain \\
$P_{i j}^{(k)}$ & Power used by user $i$ in the channel $k$ of BS $j$ \\
$p_{t}(k)$ & Probability of event $\varepsilon$ \\
& $P_{r}\left(\right.$ user $i$ attempts to reserve $i$ transmits using $\left.j^{(k)}\right)$ \\
&
\end{tabular}


$\bar{R}_{i j}^{(k)} \quad$ Average data rate for user $i$ in the channel $k$ of BS $j$

$\gamma(h) \quad$ SINR function of channel power gain $h$

$\bar{T}_{i j}^{(k)} \quad$ The average throughput for user $i$ in the channel $k$ of BS $j$

$\tilde{h}_{i j}^{(k)} \quad$ Channel power gain from the user of channel $k$ of $i$-th BS to the $j$-th BS

$f_{i j}^{(k)}(h) \quad$ The probability density of channel power gain of channel $k$ of user $i$ to BS $j$

$p_{a, i j}^{(k)} \quad P_{r}\left(\right.$ user $i$ attempts to reserve $\left.j^{(k)}\right)$

$p_{t, i j}^{(k)} \quad P_{r}\left(\right.$ user $i$ transmits using $\left.j^{(k)}\right)$

$P_{\text {noise }} \quad$ The power of noise

$r_{i}(n) \quad$ The achievable data rate of user $i$ in $n$-th time period

$\bar{R}_{i}(n) \quad$ The average data rate of user $i$ before $n$-th time period calculated according to window size WIN 


\section{Chapter 1}

\section{Introduction}

\subsection{Thesis Problem Statement and Motivation}

Since the introduction of cellular networks, scheduling and radio resource management are taken for granted as the sole responsibility of BSs (Base Station). This works well for the 2nd generation (2G) cellular networks (such as GSM and CDMA) where voice-centric services dominate. Later, with the trend from voice service to data service and network evolution from $2 \mathrm{G}$ to $3 \mathrm{G}$ to $4 \mathrm{G}$ (LTE), this poses very high demand for BSs. To schedule and make use of radio resources efficiently, BSs will make decisions based not only on all potential users' instantaneous CSI (Channel State Information) but also on the stored historic data. This is very burdensome and sometimes impossible due to the time constraints if the number of users is very large. On the other hand, mobile data services will surely account for a large percent of data volume in future cellular networks. Many emerging new mobile data services, such as M2M (Machine to Machine), mobile sensor network, and telemedicine, will have great advancements in the near future. These mobile data services may not be suitable for the current scheduling modes and exhibit some characteristics of their own. For example, for some M2M services, although the number of users is very large, their data transfer is delay insensitive, spontaneous and mostly from terminals 
to BSs (uplink). Using current scheduling mode would result in a waste of resources to some extent. Due to above reasons, a single centralized scheduling may not be a good or universal solution. With the advance of terminal capability (CPU, memory, etc.), distributed scheduling with the assistance of BSs seems to be a potential way to fit for these looming scenarios.

The thesis aims on investigating the possible ways for distributed scheduling with the assistance of BSs:

1) performing the theoretical analysis and comparison of the distributed scheduling and centralized scheduling;

2) developing algorithms for the cooperation of terminals and BSs in distributed scheduling;

3) creating a framework for distributed scheduling.

\subsection{Thesis Contributions}

To the best of our knowledge, the novelties of the thesis are listed as follows:

1) We propose a distributed scheduling framework for uplink data transfer by the cooperation of BSs and terminals: terminals calculate and send criterion values to $\mathrm{BSs}$ for making arbitration. This framework can be extended to other scenarios in the future to achieve different goals.

2) We propose a scenario of common spectrum, multi-channel and multi-cell network without cell boundaries for exploiting spatial diversity and improving performance of cell border users. 
3) Mathematical analysis of multiuser diversity and proportional fairness approximation in the scenario of common spectrum, multi-channel and multi-cell networks.

4) We proposed an algorithm for achieving maximum sum throughput while controlling the number of control resources.

5) We propose an algorithm for proportional fairness approximation with tradeoff between fairness and throughput while controlling the number of control resources.

\subsection{Thesis Organization}

Chapter 2 serves as an introduction to scheduling in wireless networks. Literature review for distributed scheduling or random access is also presented. Based on these literature surveys, we come up with our research proposals. Chapter 3 describes a detailed system model. Chapter 4 is mainly for mathematical analysis for our research proposals. For sum throughput maximization, both homogeneous and heterogeneous channel models are investigated. Chapter 5 presents a detailed analysis of simulation and numerical results. Chapter 6 discusses some of implementation issues for application of our proposed framework. Chapter 7 highlights the conclusions of the thesis and proposes the possible future research directions. 


\title{
Chapter 2
}

\section{Overview of Scheduling in Wireless}

\author{
Networks
}

\subsection{A Brief Introduction of Medium Access Con- trol Protocols}

In OSI model, the Medium Access Control (MAC) sub-layer is located in the lower Data Link layer [2]. It does not provide too much help for the point-to-point unicast networks, but for those networks where limited communication channels are contended for or shared by multiple users, MAC plays a key role in facilitating communications between users and networks.

The basic function of the medium access control is to schedule packet transmission under some policies (e.g., fairness, throughput maximization) among multiple users that share the same channels. The design of medium access control is highly related to the characteristics of communication channels used in the system. A typical example can be shown by the discrepancy of medium access control used in Ethernet and WLAN. Comparing to the wireless medium, the wired channel is much more stable and packet error in the wired network is mostly caused by collision. For this reason, the MAC protocols for wired networks are relatively simple. For example, 
the Ethernet (802.3) uses a protocol called Carrier Sense Multiple Access/Collision Detection (CSMA/CD) to manage collisions. CSMA/CD is a type of contention protocol that defines how to respond when a collision is detected, or when two devices attempt to transmit packages simultaneously. Ethernet allows each device to send messages at any time without having to wait for network permission. Thus, there is a high probability that devices may try to send messages at the same time. After detecting a collision, each device that is transmitting a packet delays a random amount of time before re-transmitting the packet. If another collision occurs, the device waits twice as long before trying to retransmit [3].

In comparison, WLAN adopts a protocol called Carrier Sense Multiple Access/Collision Avoidance (CSMA/CA) to manage collisions. This is derived from CSMA/CD (Collision Detection). The main difference is the collision avoidance : in Ethernet, the transceiver has the ability to listen while transmitting and so to detect collisions (within a wire all transmissions have approximately the same strength). But this is not the case in WLAN: even if a radio node could listen to the channel while transmitting, the strength of its own transmissions would mask all other signals on the air. So, the protocol can't directly detect collisions like with Ethernet and can only try to avoid them. In CSMA/CA, as soon as a node receives a packet that is to be sent, it checks to be sure the channel is clear (if the receiver sends CTS (Clear To Send) to the sender). If the channel is clear, then the packet is sent. Otherwise, the node waits for a randomly chosen period of time, and then checks again to see if the channel is clear. This period of time is called the backoff factor, and is counted down by a backoff counter. If the channel is clear when the backoff counter reaches zero, the node transmits the packet. Otherwise the backoff factor is set again and the process is repeated [4].

As another example, the token ring network (802.5, FDDI) provides distributed contention-free channel access to the users that share the same medium [5]. In such 
a network, all users are connected in a ring. The token is essentially a short control packet circulating around the ring infinitely. If a user is ready to transmit it needs to get the token first. After the token is got, the user holds the token and transmits the data packet to the ring. Every other user should check the packet header and verifies whether it is the receiver when the packet flows by. The intended receiver copies the packet into its buffer without removing it, so the packet keeps circulating. When the packet traverses back to the sender after one round, the sender is responsible for removing the packet and releasing the token to the ring again.

With the development of telecom industry and space exploration, many MAC protocols have been designed specially for certain scenarios and applied in all kinds of fields.

\subsubsection{Problems and Challenges in Wireless Medium Access Control}

Unlike wired communications, wireless communication offers great freedom of mobility and with the help of network infrastructure, users can get access to the network anytime, anywhere; That is why wireless communications develop so quickly these years. But this also poses many challenges caused by the characteristics of wireless channels. In this section, we review the unique properties of the wireless medium and the related problems caused in the MAC protocol design.

\section{- Channel fading and signal loss}

In wireless systems, it is the electromagnetic wave that is used to carry information from sender to receiver. Most communications happen in environments surrounded by buildings, trees or moving objects. When the electromagnetic wave propagates in all directions through these objects, it will experience three 
basic mechanisms of radio wave propagation: reflection, diffraction and scattering. These mechanisms will cause signal distortion, fades and loss, resulting in the variation of the received signal power from time to time. Multipath fading is one of the major reasons for channel instability in wireless networks ([6], [7]).

\section{- Interference}

Another cause for poor channel quality comes from the interference by the transmission of nearby users because of frequency reuse in current networks. To reduce the side effects of channel fading, a sender usually transmits with strong power to maximize the received power at the receiver side. This generates bigger interference to the users in its vicinity and poses challenges for the receiver to decode the signal effectively. As a consequence of multipath propagation and interference, the wireless medium is much more vulnerable to error. The bit error rate (BER) in the wireless networks is typically more than $10^{-3}$ and often in long burst errors, while the BER is less than $10^{-6}$ in wired networks. To measure the channel quality of the wireless links, signal-to-noise ratio (SNR) or signal-to-interference and noise ratio (SINR) is used in the physical layer. A higher SNR/SINR implies better channel quality and less bit error rate. When a station detects its SNR/SINR drops to certain threshold, the channel is considered noisy and data transmission should be given up.

\section{- Hidden/exposed terminals}

In most wireless data networks, the transmission range of a station is usually short. Therefore it is unrealistic to assume the full connectivity of the system, that is, not all stations in a network are able to hear from each other. A station can only transmit to or receive from another station whose physical location is within its transmission range. This presents unique problems to the design of medium access control: the hidden/exposed terminal (station) problems. The 
hidden terminal problem is caused when two senders can not hear each other because of distance, they might transmit simultaneously and cause collisions at the receiver. The exposed terminal problem happens when the two senders may defer the transmission because they can hear each other but actually their transmission will not cause collision at two receivers because these two receivers will not be influenced because of long distance between them.

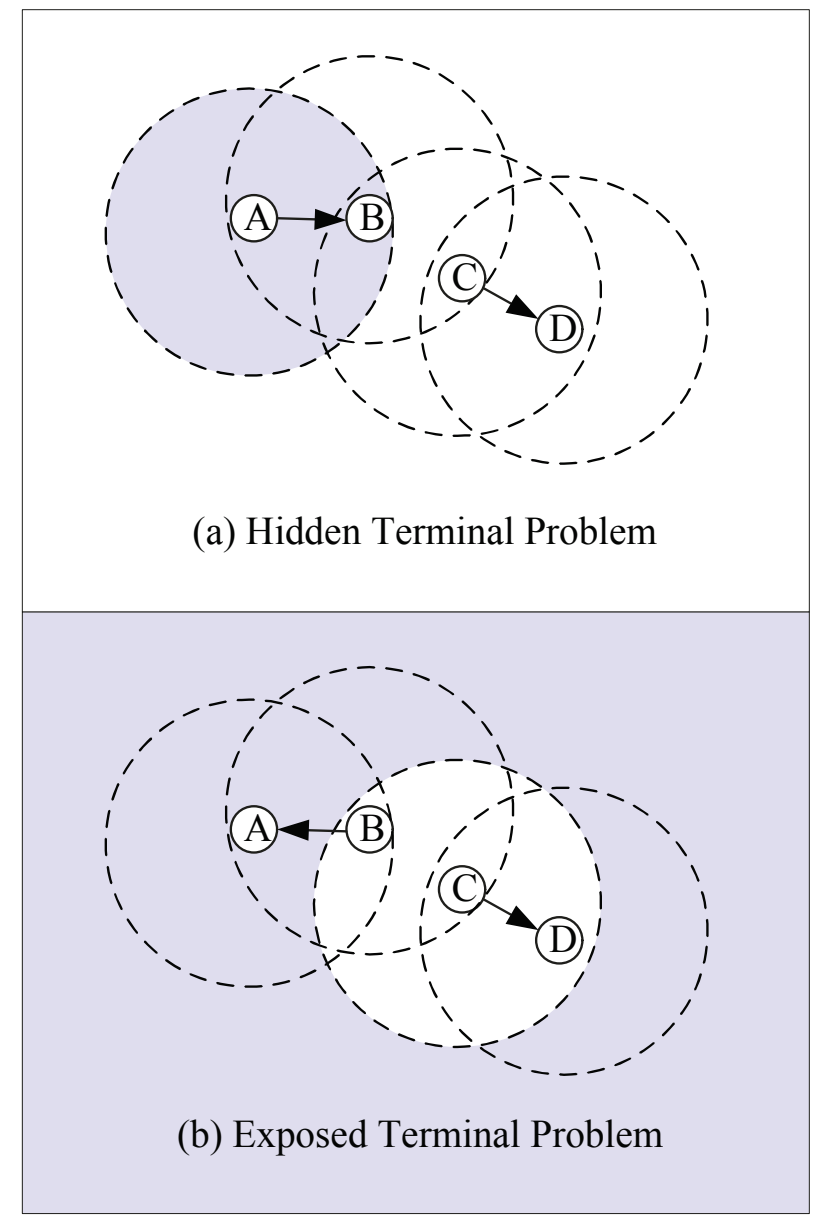

Figure 2.1: Hidden terminal problem and exposed terminal problem [1].

In Figure 2.1(a), Station $\mathrm{C}$ is within the transmission range of Station B but not in A's transmission range. When Station A is transmitting to Station B, $\mathrm{C}$ could not hear. Therefore, Station $\mathrm{C}$ might initiate a transmission to $\mathrm{D}$ and 
results in a collision at Station B, known as a "hidden terminal problem". In Figure 2.1(b), Station C is transmitting to Station D, and the transmission is overheard by B. Thus B will unnecessarily defer its intended transmission to A, in order to "avoid" interference to C's transmission. However, since B is out of D's collision domain, its transmission attempt will not affect Station D from receiving packet correctly. The false transmission deferment by a neighbor of the sender is an "exposed terminal problem".

\section{- Half duplex}

Because the transmitting power is much greater than the receiving power, the wireless networks are mostly operated in half duplex manner to prevent part of the transmitted power from penetrating into the receiver path.

To address these issues and schedule channel contention efficiently, most wireless medium access control protocols incorporate collision avoidance and collision resolution techniques into their protocols. The major collision avoidance algorithms include physical carrier sense, RTS/CTS (Ready To Send/Clear To Send) handshaking and multichannel control, etc. The major collision resolution algorithms include splitting algorithms and random backoff algorithms, etc. ([8], [9]).

The physical carrier sense algorithm used in the wireless networks is originated from the original CSMA. In the non-persistent CSMA, if the channel is busy, a station waits a random time before it senses the channel again. In the p-persistent CSMA, when the channel is idle, a station transmits packet with certain probability $p$ only. These designs reduce the probabilities that multiple stations access the channel at the same time and help improve the system performance [10].

The physical carrier sense with RTS/CTS handshaking forms the CSMA/CA algorithm, which becomes the fundamental idea of the WLAN MAC design. 
Multichannel with stand alone control channel or busy-tone is another way to improve system efficiency and reduce channel collision. The idea is splitting the channel into several sub-channels and performing the busy-tone (or the RTS/CTS handshaking) in the control channel. Theoretically, this can reduce the collision probability and improve the performance ([11], [12], [13]).

The collision resolution algorithms, especially the random backoff algorithms, are usually combined with collision avoidance algorithms in MAC design. The basic idea of backoff algorithm is to require a user to wait a random period of time before it attempts to transmit again. The splitting algorithm is to divide users into small subgroups by using a series of mini slots before retransmission, and only a user under certain rules can transmit. In this way, the simultaneous transmissions are minimized since only stations in one subgroup can transmit at a given time [14].

\subsubsection{Classification of Wireless MAC Protocols}

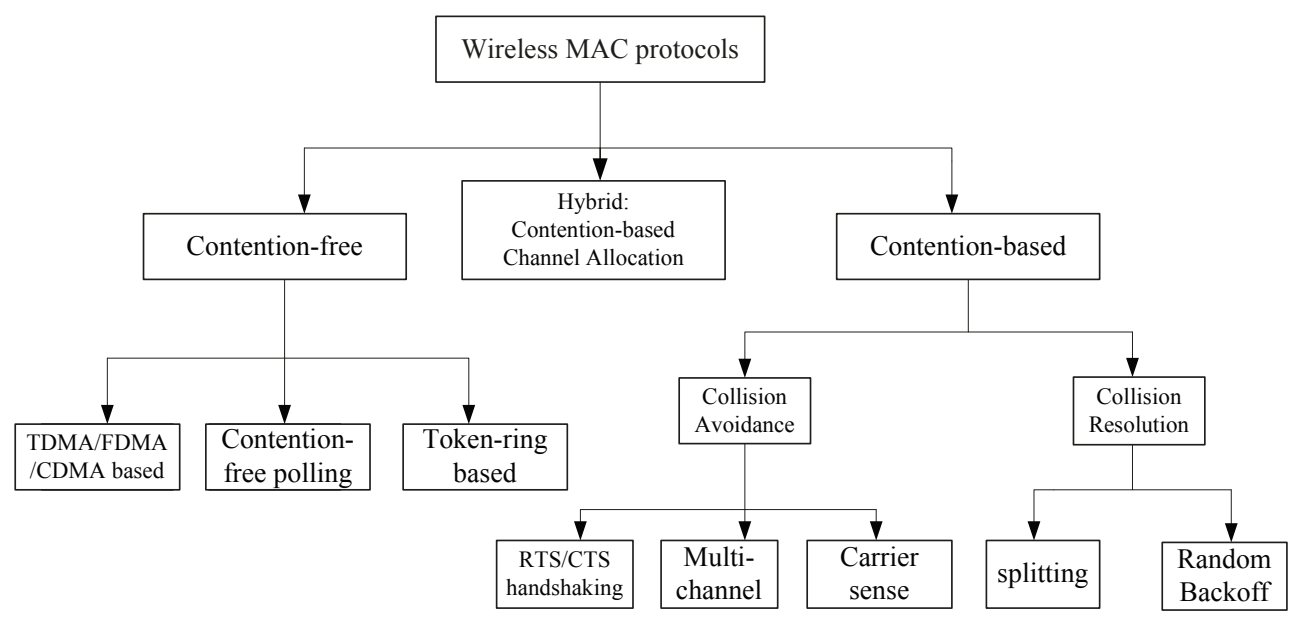

Figure 2.2: Classification of wireless MAC protocols.

Wireless MAC protocols can be broadly categorized as contention-based, contention-free and hybrid, depending on the channel access mechanisms [1]. 
Contention-free MAC protocols such as TDMA/CDMA/FDMA based protocols allow each station to access the channel by a predetermined time slot, code sequence or frequency band. Since the channel access schedule is fixed, the transmission is guaranteed to be collision-free. The polling scheme can be used as an alternative contention-free algorithm. It requires the centralized controller to poll each station at a time, and the polled station is allowed to transmit data packets within the permitted duration. All these algorithms require the coordination of a central controller, such as a base station or an access point. The token-ring based wireless MAC protocol provides a distributed way to offer contention-free channel access. More specifically, a token is circulated among all stations in the network in a round-robin fashion. A station can only transmit data when it gets the token.

The contention-based MAC protocols allow stations to access the channel randomly when they are ready to transmit. As a result, packet collision is inevitable. There are many proposed algorithms and protocols to utilize the channel more efficiently. The contention-based algorithm provides great flexibility to the network, since there is no predetermined schedule to follow. A contention-based protocol usually works well when the network load is light. When the network load is heavy, many stations attempt to access the channel simultaneously and they may experience continuous collisions, causing the system performance downgraded. In contrast, the contention-free algorithm defines rigid channel access rules on how to schedule data transmission. When a network load is light, there is bandwidth waste since some stations that are given the resources may sometimes have no data to transmit. But a contention-free protocol often is superior in heavily loaded networks, since stations do not need to waste time and bandwidth to contend the channel, and transmission delay is bounded.

A hybrid MAC protocol can combine contention-based and contention-free allocation strategies to adapt to changes in network conditions such as traffic load, 
density, or topology changes. For example, contention under lightly loaded traffic conditions, allocation under highly loaded traffic conditions.

\subsubsection{Performance Metrics of Medium Access Control Pro- tocols}

To understand the various MAC protocols that have been proposed by far, it is important for us to know how to evaluate and compare the performance of MAC protocols. A set of commonly accepted criteria, therefore, are necessary for studying wireless medium access controls [15].

\section{- Channel throughput}

Channel throughput is the portion of channel capacity used for transmitting user data. Channel throughput can be represented either in ratio or by the amount of bandwidth consumed in data transmission such as spectral efficiency. The part not directly used for sending data but just for facilitating is considered as overhead. Obviously, when data collision and transmission error happen, this will result in significant bandwidth waste. Control packets, such as RTS, CTS and ACK frames are designed to effectively reduce the probability of data collision. But they would also decrease the system throughput, since these control packets consumes the scarce bandwidth to transmit. In multi-channel protocols, there are usually one or more separate sub-channels dedicated for transmitting control packets, which negatively impact the channel throughput as well. How to balance between reducing the packet collision and the overhead issues is a key issue to be considered in MAC protocol design.

\section{- Fairness}

For contention-based wireless networks, fairness is an important issue among 
stations of equal priority. This is also of practical requirements in commercial telecom networks. Fairness offers randomized channel access when multiple stations compete for the channel at the same time. However, unbiased bandwidth allocation is not easy to achieve in the distributed networks since all users may not have other users' channel information. Achieving fairness and avoiding starvation is another design goal of medium access control.

\section{- Power consumption}

Power consumption is another important design goal in medium access control, especially for mobile ad-hoc networks and wireless sensor works where battery replacement could be expensive or even prohibitive. Many MAC protocols allow stations to switch to power-saving mode when they don't need to transmit or receive packets. Also, power allocation is also important to the performance of user since it is closely related with the interferences caused to adjacent users.

\section{- QoS provisioning}

The convergence of voice, video and data brings tremendous needs for wireless networks with QoS provisioning. Providing QoS in distributed wireless data networks is not trivial, because real-time transmission is highly delay-sensitive. QoS provisioning in the MAC sub-layer is traditionally performed with the coordination of central controllers to allocate radio resources wisely and efficiently. But this is not the case in the distributed wireless networks since no centralized nodes will exist in these networks. It is critical for a QoS aware MAC protocol to offer at least certain degrees of packet prioritization and delay guarantee [16]. This issue is sometimes connected with fairness issue since some users with high priorities may need to be specially provided with high QoS. 


\subsection{Discussion on Centralized Scheduling and Challenges}

Another kind of categorization of wireless MAC is based on the roles of central controllers in different network architectures: distributed and centralized [15].

Centralized scheduling plays a dominant role in current cellular networks to fully utilize limited radio resources and it provides users contention-free access to radio resources. This usually implies running complex algorithms and large scale computation burden on BSs especially when the number of users or channels is considerably large. Centralized scheduling may be competent for handling a small number of users while it may have difficulties in coping with a large number of users or fast fading channels. When there are a lot of users contending for channels, it is hard for BSs to get all users latest channel information and run the related algorithms to appropriately schedule users data transfer, or it may exceed the coherence time of the channel and leads to the obsoleteness of channel information. On the other hand, mobile terminals are becoming more and more powerful with the advance of chipset technology and they have enough processing power to complement the scheduling in Base Stations. This leads us to investigating the distributed way of scheduling by the cooperation of BSs and terminals, which is generally regarded as the way of random access methods or the contention-based Medium Access Control type.

\subsection{Distributed Scheduling/Random Access Dis- cussion and Literature Review}

Random access has been used as a basic way for media access control since the birth of ALOHA in 1970s. Wired Local Area networks (LANs) use carrier sensing 
multiple access with collision detection (CSMA/CD) protocol, while Wireless Local Area networks (WLANs) use a revised version named as collision avoidance (CSMA/CA) protocol. These protocols are well suitable to the local area networks where unlicensed spectrum is used as one channel shared by all users, and some extensions to these protocols were studied for the scenarios of multi-channel WLAN [17] and multi-channel cellular environments [18]. An OFDMA-based multi-channel CSMA/CA protocol is studied in [17], and it has been found that a lower collision probability and higher channel utilization can be achieved when compared with the single-channel CSMA/CA protocol. In [18], a fast retrial algorithm is introduced to improve throughput and reduce access delay in multiple uplink channels of OFDM network. Whenever a collision happens, the user jumps to a channel with different carrier frequency in next time slot. In this way, randomness is introduced in both time and frequency domain.

However, the above papers pay little attention to the role of wireless channels which is especially important in cellular networks. Hence, they are not appropriate for cellular networks. Wireless channels are characterized by fading effects which are caused by the destructive and constructive summation of multipath signals. This may lead to high dynamic range in the amplitude of the received signals in time domain and serious distortion of original signals in frequency domain. Fortunately, fading effects of wireless channels are not always harmful and they can be exploited by some technologies such as MIMO to achieve higher capacity. Multi-user diversity is also an example for using fading effects of wireless channels. The main idea is to select those users with better channel state information for data transfer. In this way, higher channel capacity can be achieved. The notion of multi-user diversity was originally studied in [19], where optimal uplink power control scheme in the scenario of multiuser in a single cell was presented: users or terminals feedback their channel station information to BSs and adjust their transmit power based on the 
instructions from BSs, only users with best channel can transmit and the capacity increases significantly as the number of users increases. Note that the idea in [19] is based on centralized scheduling. A practical system capitalizing on multi-user diversity with centralized downlink scheduling is Qualcomm's High Data Rate (HDR) system (1xEV-DO) [20].

Random uplink access with multi-user diversity has been extensively investigated in several papers such as [21]- [22]. A decentralized medium access control protocol called channel-aware ALOHA is presented in [21], where each user transmits only if its channel power gain is above a pre-determined threshold that is chosen to maximize the probability of successful transmissions. The performance of this protocol in the scenario of a single uplink channel in a single BS is analyzed. It shows that without centralized scheduling we can still exploit multi-user diversity gains. The ratio of the throughput of this protocol to that of a centralized scheduler is $1 / e$ when the number of users goes to infinity and the loss is caused by the collision of different users contending for the same channel. This scenario is further investigated in [23], and it proposes that this kind of binary scheduling (that is, users transmit whenever their channel power gain surpasses a threshold, otherwise keep silent) can maximize sumthroughput for homogeneous systems (this means that users have the same channel distribution), and maximizes the sum of the logs of the average throughputs for heterogeneous systems (this means that users may have different channel distribution) while providing proportional fairness $(\mathrm{PF})$. Multi-channel random access is proved to be superior to single-channel random access under the same bandwidth and transmission power by exploiting multicarrier diversity [24]. It is also found in [25] that a proposed Channel Aware Multi-Carrier Random Access scheme (CAMCRA) is much more robust to imperfect channel information than is random access in a single carrier. An opportunistic clustered-OFDM-based multi-channel Aloha (OMC-Aloha) is 
proposed in [26], where multi-channel in a single BS scenario is studied. A user transmits if it has at least $m$ channels with gains greater than a pre-determined threshold. Similar to the results in [21] and [23], it is also shown that the OMC-Aloha is asymptotically order optimal, in the sense that the only performance loss compared to the optimal centralized scheduling is due to the contention inherent in random access. OMC-Aloha can also provide the PF by maximizing the sum of the logarithms of users throughput for heterogeneous systems which is similar to the result in [23].

Multi-channel random access in mobile ad hoc networks is investigated in [27]. It proposes a scheme named as Decentralized Optimization for Multichannel Random Access (DOMRA). The scheme consists of three steps: (1) neighborhood information collection: collecting broadcast information to get the number of interfering neighbors; (2) transmission control of the MAC layer based on the instantaneous channel state information: calculation of channel gain threshold based on the neighborhood information and then use it for transmission control; (3) power allocation of each traffic flow on each subchannel: capability-limited water-filling algorithm is proposed.

Recently, multi-user diversity in the scenario of multi-BS is investigated in [28]. In this paper, a Distributed Opportunistic Scheduling (DOS) protocol is proposed. Two thresholds are defined: one is for channel power gain $\eta_{t r}$ and the other is for aggregate channel power gain of inter-cell interference $\eta_{I}$. Each of BSs will randomly select users within its cell coverage who meet those two thresholds (i.e. channel power gain is greater than $\eta_{t r}$ and aggregate channel power gain of inter-cell interference is less than $\eta_{I}$ ) and selected users then start data transmission.It is proved that the achievable sum-rate scales as $K \log (\mathrm{SNR} \log N)$ in a high signal-to-noise ratio (SNR) regime, if the total number of users in a cell, $N$, scales faster than $\operatorname{SNR}^{\frac{K-1}{1-\epsilon}}$ for a constant $\epsilon \in(0,1)$. Also, DOS protocol can be easily extended to multi-carrier systems (i.e. multi-channel systems).

It has been shown in [21] and [23] that collision by different users contending 
the same channel is the main reason for throughput loss compared with centralized scheduling. For this reason, some schemes for contention resolution are explored in [29], [22]. A splitting algorithm is proposed to resolve collisions over a sequence of mini-slots, and determine the user with the best channel [29]. It also shows that the average number of mini-slots required to find the user with the best channel is less than 2.5 independent of the number of users or the channel distribution [29]. While in [22], a scheme called Channel-Aware Distributed Medium Access Control (CAD-MAC) is proposed for wireless ad hoc networks or sensor networks. Each frame slot is divided into contention and transmission periods, and resolution of collisions is conducted in the contention period, then winners transmit in the transmission period. For the contention among links with the same transmitter, namely, Type-I, resolution is made by transmitters themselves locally with fairness taken into consideration. Type-II, links with different transmitters, should be resolved afterwards. Different signals are used during contention resolution until one link is selected within each local area and its interferers are informed to keep silent in the current frame slot [22].

\subsection{Summary and Research Proposals}

The above literature review can be summarized as the Table 2.1:

All the literature above except [28] focuses on a single BS scenario with single or multiple channels, where random access based on the channel power gain threshold is adopted and base stations do not involve in the scheduling. Although the multi-BS with single or multiple channels scenario is studied in [28], it does not fully exploit spatial diversity provided by multiple BSs. Here, by spatial diversity we mean that users can select not only channels with different carrier but also channels belonging to different BSs. We would exploit multi-user diversity as well as spatial diversity in 
Table 2.1: Summary of literature review.

\begin{tabular}{|c|c|c|c|c|c|c|c|}
\hline \multirow{2}{*}{ References No. } & \multirow{2}{*}{ Single-Channel } & \multirow{2}{*}{ Multi-Channel } & \multirow{2}{*}{ WLAN } & \multirow{2}{*}{ Ad hoc } & \multicolumn{2}{|c|}{ Cellular } & \multirow{2}{*}{ Channel Awareness } \\
\hline & & & & & Single-BS & Multi-BS & \\
\hline$[17]$ & & $\sqrt{ }$ & $\sqrt{ }$ & & & & \\
\hline$[18]$ & & $\sqrt{ }$ & & & $\sqrt{ }$ & & \\
\hline$[19]$ & $\sqrt{ }$ & & & & $\sqrt{ }$ & & $\sqrt{ }$ \\
\hline$[20]$ & & $\sqrt{ }$ & & & $\sqrt{ }$ & & $\sqrt{ }$ \\
\hline$[21]$ & $\sqrt{ }$ & & & & $\sqrt{ }$ & & $\sqrt{ }$ \\
\hline$[22]$ & & $\sqrt{ }$ & & $\sqrt{ }$ & & & $\sqrt{ }$ \\
\hline$[23]$ & $\sqrt{ }$ & & & & $\sqrt{ }$ & & $\sqrt{ }$ \\
\hline$[24]$ & & $\sqrt{ }$ & & & $\sqrt{ }$ & & $\sqrt{ }$ \\
\hline$[25]$ & & $\sqrt{ }$ & & & $\sqrt{ }$ & & $\sqrt{ }$ \\
\hline$[26]$ & & $\sqrt{ }$ & & & $\sqrt{ }$ & & $\sqrt{ }$ \\
\hline$[27]$ & & $\sqrt{ }$ & & $\sqrt{ }$ & & & $\sqrt{ }$ \\
\hline$[28]$ & & $\sqrt{ }$ & & & & $\sqrt{ }$ & $\sqrt{ }$ \\
\hline$[29]$ & $\sqrt{ }$ & & & & $\sqrt{ }$ & & $\sqrt{ }$ \\
\hline
\end{tabular}

a multi-channel and multi-BS scenario to further increase spectral efficiency. Also, we propose that terminals be more involved in scheduling and power control which leads to distributed characteristics of these basic functions. We also propose that BSs perform the function of arbitration to fully use the network capacity instead of wasting channels due to collisions. The basic idea is like this: if two terminals transmit data at the same channel, collision will happen and neither of them will get its data transmitted through at the expense of wasting one channel. But if the BS can do arbitration, that is, it use some criterion to select one terminal and tell it that it can transmit using this channel, then the channel is fully utilized. In this way, we can avoid the throughput loss caused by collision. 
Table 2.2: Comparison of our work with literature.

\begin{tabular}{c|c|c}
\hline \hline Novelties & Our Work & Shin'12 ([28]) \\
\hline Two Thresholds & $\sqrt{ }$ & $\sqrt{ }$ \\
\hline BS Diversity & $\sqrt{ }$ & \\
\hline Throughput and Overhead Tradeoff Analysis & $\sqrt{ }$ & \\
\hline Criterion Value Computation & $\sqrt{ }$ & \\
\hline Proportional Fairness Discussion & $\sqrt{ }$ & \\
\hline Framework Discussion & $\sqrt{ }$ & \\
\hline
\end{tabular}

Our research is among the first few to investigate this field systematically. Roughly compared with the work in [28], our work greatly expanded and enriched the research areas in this field as shown partly in Table 2.2. The notions in the table will be discussed in the following chapters. 


\section{Chapter 3}

\section{System Model Description}

\subsection{Introduction}

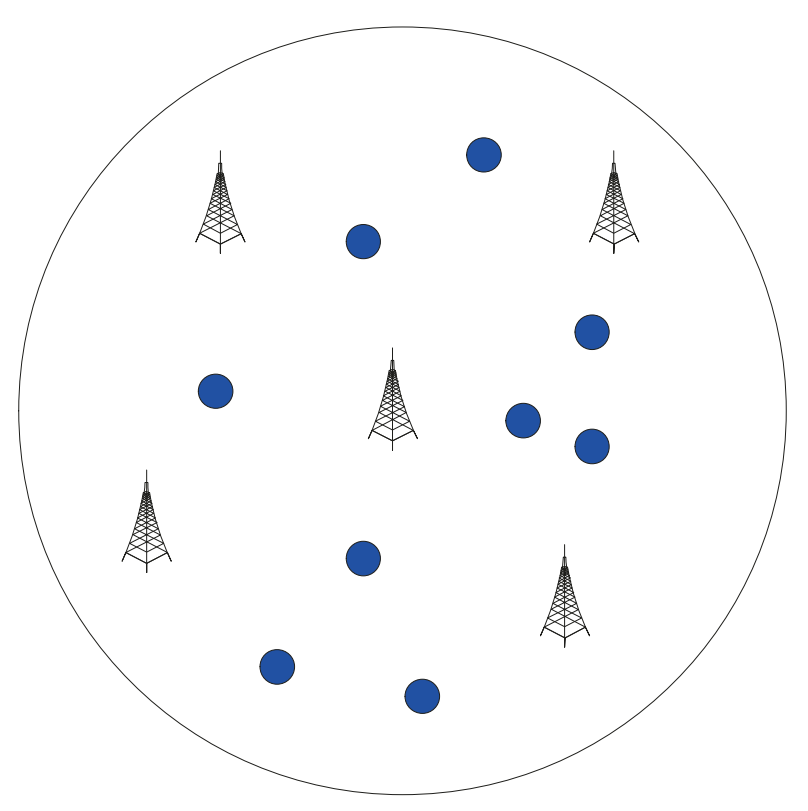

Figure 3.1: System model.

In the current cellular network, mobile users can only be served by one BS at one time. We extend this kind of a single cell operation mode to a multi-cell operation mode where each user can select its serving BS based on the quality of the links to different BSs (Figure 3.1). In this way, we can exploit spatial diversity to some extent. 
This may be favorable for those users located at cell border areas if they can select the "best" BS to serve them. The purpose of this extension also serves to simplify radio network planning. Instead of splitting a certain area to several independent cells, we try to analyze the possibility of using cell clusters to serve terminals.

The main points of this multi-cell system model are summarized as follows:

1) $N$ BSs are located within a certain area, each has $L$ channels.

2) $M$ users are uniformly distributed in this area and each user has an infinity number of packets to send, that is, a full queue model.

3) Every user only knows its own CSI based on the estimation of pilot signals from all BSs. For example, for user $i$, the channel power gains for channel $k$ of $N$ Base Stations are $h_{i 1}^{(k)}, h_{i 2}^{(k)}, \cdots h_{i N}^{(k)}$.

\subsection{Distributed Scheduling Framework}

As discussed in Chapter 2, we propose that terminals can be more involved in scheduling with the assistance of BSs. Also, we would like that this joint scheduling by the cooperation of terminals and BSs can support different scheduling schemes such as sum throughput maximization and proportional fairness. To facilitate this, a clear functional partition of terminals and BSs is necessary. We propose a distributed scheduling framework which defines the basic functions of terminals and BSs and the workflow of scheduling procedures.

The proposed framework can be illustrated by the following diagram: 


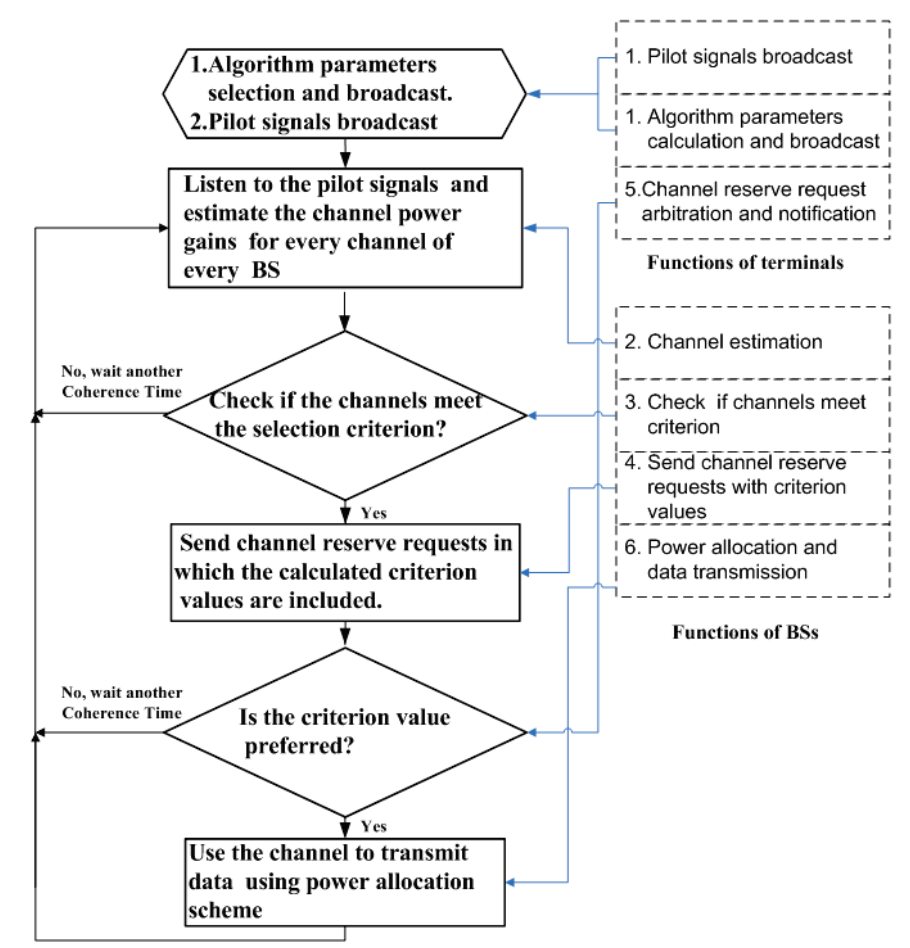

Figure 3.2: Distributed scheduling framework flow chart

The basic workflow of this distributed scheduling framework is as follows:

1) BSs broadcast pilot signals for channel estimation and algorithm parameters for scheduling to terminals. The algorithm mentioned here are used by terminals and BSs to achieve different scheduling goals such as sum throughput maximization;

2) Terminals estimate channels of every BS;

3) Based on the results of channel estimation, terminals will check if channels meet certain criteria based on algorithm parameters;

4) If certain criteria are satisfied, terminals send channel reserve requests to BSs with criterion values included; 
5) BSs will get channel reserve requests from different terminals and arbitrate which terminal is preferred. Then send notification to the related terminals, which means that the related terminals can use the channel for data transfer;

6) If the terminal gets the notification, it will allocate power and make data transfer. Otherwise, terminals will keep silent and repeat this procedure again.

This framework is not only applicable to the scheduling goals of sum throughput maximization and proportional fairness but also to other scheduling goals as well. For different scheduling goals, we just need to use appropriate algorithms.

A possible implementation of this framework is show in Figure 3.3.

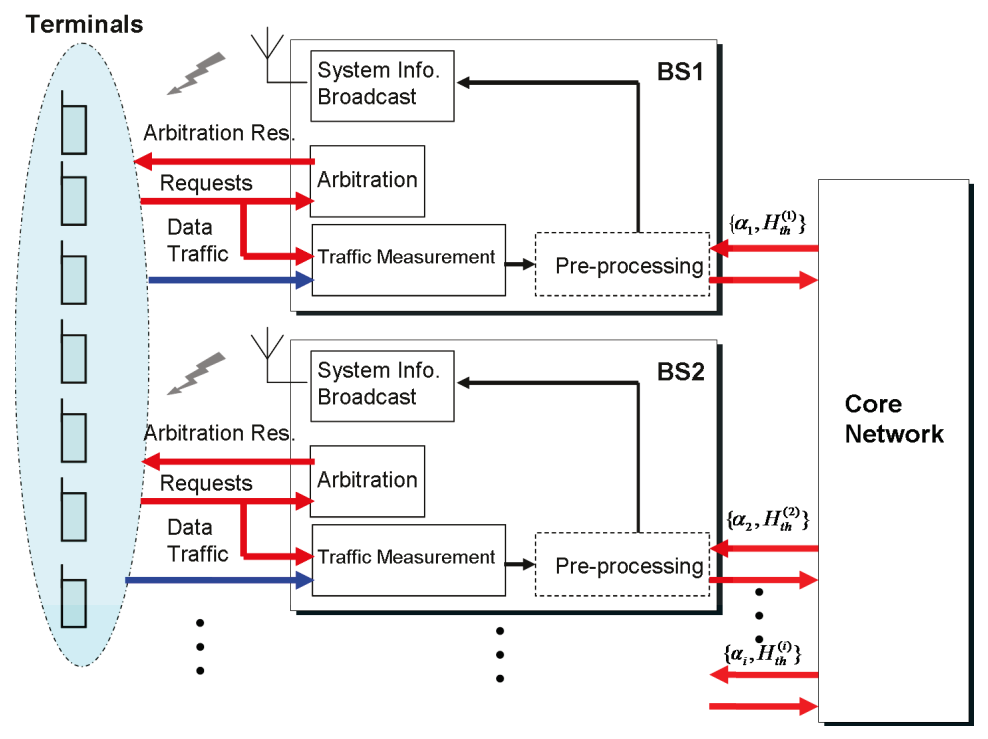

Figure 3.3: A possible implementation block diagram.

In real case, network conditions such as the number of users, traffic load distribution among BSs are always changing. To get sound performance, we need the network to be adaptive to these changing conditions by adjusting the related algorithm parameters dynamically. As showed in Figure 3.3, core network nodes can be 
used to coordinate different BSs and calculate algorithm parameters for different BSs based on operators' policies.

The basic idea of this adaption is as follows: 1) BSs will measure the user data traffic and analyze channel reserve requests to provide this historic data to core network nodes; 2) core network nodes will based on policies and the historic information from BSs to decide the suitable algorithm parameters for each BS; 3) BSs will broadcast the updated algorithm parameters as system information to terminals.

\subsection{Proposed Distributed Scheduling Algorithms}

Based on the framework discussed above, we propose two algorithms for two scenarios: sum throughput maximization and proportional fairness.

\subsubsection{Algorithm for Scenario I: Sum Throughput Maximiza- tion}

To achieve the goal of sum throughput maximization, we need to exploit multiuser diversity. This means that we should select users with best channel conditions to make data transfer. For centralized scheduling, BSs will try to collect all users' CSI or other parameters then allocate channels to those users with better channel conditions. This will obviously consume a lot of control overhead. This can be improved by using our distributed scheduling methods. First, among all the channel information BSs collect from terminals, BSs only need to select among those terminals whose channel conditions are above a certain level. We can call this kind of terminals as candidate users. In this sense, BSs does not need to know all users' channel conditions. If the number of terminals is large, probabilistically there are always a few candidate users whose channel conditions are above a certain level. This motivates us to set certain thresholds to all the terminals and only those terminals whose channel conditions 
are above these thresholds can compete for the channels. In this way, we can not only select the terminals with better channel conditions but also effectively reduce the control overhead needed. Based on the above analysis, we propose the following algorithm for achieving sum throughput maximization:

\section{Algorithm I for Channel Access at Terminals}

For every terminal $i(i=1,2, \cdots, M)$, CSI of channel $k(k=1,2, \cdots L)$ of $N$ BSs is estimated as $h_{i 1}^{(k)}, h_{i 2}^{(k)}, \cdots h_{i N}^{(k)}$.

If $\max \left(h_{i l}^{(k)}\right)=h_{i j}^{(k)} 1 \geq H_{t h},(l=1,2, \cdots N)$

Then If $h_{i l}^{(k)} \leq \alpha H_{t h}(l \neq j)$

Then (1) Calculate the criterion value $\frac{h_{i j}^{(k)}}{\sum_{l \neq j} h_{i l}^{(k)}}$

(2) Send Channel Reserve Request to BS $j$

(3) Wait for notification, if got, transmit using channel $k$

Else Wait for next Coherence Time, check new estimated channel info.

The basic idea of Algorithm I is as follows:

1) Each user will listen to the broadcast channel for the value of related parameters used in the algorithm and estimate the channel power gain based on the pilot signals.

2) For every channel, each user will select the greatest channel power gain from $N$ BSs and compare it with a threshold $H_{t h}$ (which is broadcast by BSs). If it is greater than the threshold and other non-maximum channel power gains is less than certain value $\left(\alpha H_{t h}, \alpha\right.$ is called attenuation factor and is also broadcast by BSs), the user will try to reserve the channel of BS that has the

\footnotetext{
${ }^{1}$ Note: terminal $i$ will first select the BS based on the largest channel power gain, BS $j$ is selected in this case because $h_{i j}^{(k)}$ is the largest one among $N$ BSs.
} 
greatest channel power gain by sending channel reserve requests in control channels.

When $H_{t h}$ is at low region, the value of $\alpha H_{t h}$ will be small which means that the number of candidate users is mostly decided by the value of $\alpha$. Because of multiuser diversity, the sum throughput will also be small; When $H_{t h}$ is at high region, the value of $\alpha H_{t h}$ will be large which means that the number of candidate users will be mostly decided by the value of $H_{t h}$. High value of $H_{t h}$ will also restrict the number of candidate users, hence the sum throughput will also be small with the increase of $H_{t h}$. Based on the above analysis, the peak value of sum throughput should be achieved in the middle range of $H_{t h}$.

3) Base Stations will receive channel reserve requests from different users. They will do arbitration, that is, selecting the user with the largest criterion value (in case of equal values, random selection is used) and inform the user, which means that the user can transmit using this channel. 
After BSs' arbitration, we can get $L N$-dimensional channel power gain square matrices as follows:

$$
\left(\begin{array}{cccc}
\tilde{h}_{11}^{(1)} & \tilde{h}_{12}^{(1)} & \ldots & \tilde{h}_{1 N}^{(1)} \\
\tilde{h}_{21}^{(1)} & \tilde{h}_{22}^{(1)} & \ldots & \tilde{h}_{2 N}^{(1)} \\
\vdots & \vdots & \ddots & \vdots \\
\tilde{h}_{N 1}^{(1)} & \tilde{h}_{N 2}^{(1)} & \ldots & \tilde{h}_{N N}^{(1)}
\end{array}\right)\left(\begin{array}{cccc}
\tilde{h}_{11}^{(2)} & \tilde{h}_{12}^{(2)} & \ldots & \tilde{h}_{1 N}^{(2)} \\
\tilde{h}_{21}^{(2)} & \tilde{h}_{22}^{(2)} & \ldots & \tilde{h}_{2 N}^{(2)} \\
\vdots & \vdots & \ddots & \vdots \\
\tilde{h}_{N 1}^{(2)} & \tilde{h}_{N 2}^{(2)} & \ldots & \tilde{h}_{N N}^{(2)}
\end{array}\right) \ldots\left(\begin{array}{cccc}
\tilde{h}_{11}^{(L)} & \tilde{h}_{12}^{(L)} & \ldots & \tilde{h}_{1 N}^{(L)} \\
\tilde{h}_{21}^{(L)} & \tilde{h}_{22}^{(L)} & \ldots & \tilde{h}_{2 N}^{(L)} \\
\vdots & \vdots & \ddots & \vdots \\
& & & \\
\tilde{h}_{N 1}^{(L)} & \tilde{h}_{N 2}^{(L)} & \ldots & \tilde{h}_{N N}^{(L)}
\end{array}\right)
$$

Here $\tilde{h}_{i j}^{(k)}$ is the channel estimation from the user of channel $k$ of $i$-th BS to the $j$-th BS, when $i=j$, such as $\tilde{h}_{i i}^{(k)}$, which represents the channel power gain of the user of channel $k$ of $i$-th BS. This means that diagonal elements of every matrix are the channel power gains of the actual users' and other elements in the same row are the channel users' channel power gain to other BSs for the channel (i.e., channel power gain of interference channels). For example, $\tilde{h}_{12}^{(3)}$ is the channel power gain for the interference link from the user of channel 3 of the first BS to the second BS and $\tilde{h}_{11}^{(3)}$ is the channel power gain of the communication link by the of channel 3 user of the first BS.

\subsubsection{Algorithm for Scenario II: Proportional Fairness Ap- proximation}

It is proved that a proportional fair allocation actually maximizes the sum of the logarithms of average user rates, which means that the allocation scheme is the 
solution to the following optimization problem [30]:

$$
\begin{aligned}
\operatorname{Maximize} & \left\{\sum_{i}^{M} \log \bar{R}_{i}\right\} \\
\text { s.t. } & \sum_{i}^{M} \bar{R}_{i}=C \quad(i=1,2, \ldots, M) .
\end{aligned}
$$

Where $M$ is the number of users and $\bar{R}_{i}$ is the average rate of user $i, C$ is the available channel bandwidth. In a single channel system, proportional fairness is achieved by scheduling at each time period to user $i$ according to

$$
i^{*}(n)=\arg \max _{i} \frac{r_{i}(n)}{\bar{R}_{i}(n)} .
$$

Here, $r_{i}(n)$ is the achievable data rate of user $i$ in $n$-th time period, $\bar{R}_{i}(n)$ is the average data rate of user $i$ before $n$-th time period calculated according to a predefined window size WIN.

However, it should be noted that the above result is based on the implicit assumption that the probability for every user's transmission is same. This is obviously not the case for our scenario where those users closer to BSs will certainly enjoy a higher probability of data transmission, so a revised version of PF called weighted proportional fairness (WPF) should be adopted [31]. In contrast to (3.1), the optimization problem for WPF is

$$
\begin{aligned}
\operatorname{Maximize} & \left\{\sum_{i}^{M} w_{i} \log \bar{R}_{i}\right\} \\
\text { s.t. } & \sum_{i}^{M} \bar{R}_{i}=C \quad(i=1,2, \ldots, M) .
\end{aligned}
$$


Here, $w_{i}$ is the weight for user $i$. Similarly, (3.2) is revised as

$$
i^{*}(n)=\arg \max _{i} \frac{w_{i} \cdot r_{i}(n)}{\bar{R}_{i}(n)}
$$

Using Lagrange method, it is easily to get the solution to (3.3):

$$
\bar{R}_{i}=\frac{w_{i}}{\sum_{i} w_{i}} \cdot C .
$$

For user $i$, the actual average throughput $\bar{T}_{i}$ is

$$
\begin{aligned}
\bar{T}_{i} & =P_{r}(\text { user } i \text { transmits using the channel }) \cdot \bar{R}_{i} \\
& =\frac{p_{t, i} \cdot w_{i}}{\sum_{i} w_{i}} \cdot C, \quad P_{r}(\text { user } i \text { transmits using the channel })=p_{t, i}
\end{aligned}
$$

If we let $w_{i}$ equal $\frac{1}{p_{t, i}}$, then each user will get the same average throughput. In this way, we achieve fairness among all users whether they are close to or far away from BSs, which means that (3.4) should be revised as

$$
i^{*}(n)=\arg \max _{i} \frac{r_{i}(n)}{p_{t, i} \cdot \bar{R}_{i}(n)}
$$

(3.7) can be the criterion value used in the request arbitration at BSs. Probabilistically, each user can get fairness in the long run. But two issues related to this method need to be analyzed: firstly, it's hard for a user to get the exact value of its weight, that is, the reciprocal of $p_{t, i}$; secondly, it's difficult for a user to estimate accurately the value of $r_{i}(n)$. These two values are closely related to the characteristics of other users' channels, which are generally unavailable to a single user.

In order to overcome this difficulty, we suggest using an approximation to proportional fairness. Because it is hard for a single user to calculate the interference 
caused by other users, we decouple this by using the interference caused by a single user itself to other users for estimating instantaneous rate. That is

$$
\hat{r}_{i}^{(k)}(n)=\frac{W}{L} \cdot \log \left(1+\frac{P h_{i j}^{(k)}}{N_{0} \cdot \frac{W}{L}+P \sum_{l \neq j} h_{i l}^{(k)}}\right) .
$$

In some sense, it is favorable in channel selection since the greater value for $\hat{r}_{i}^{(k)}(n)$ means the less interference it will cause to other users. Hence, (3.7) can be changed as

$$
i^{*(k)}(n)=\arg \max _{i} \frac{\hat{r}_{i}^{(k)}(n)}{\bar{R}_{i}(n)} .
$$

Here, $\bar{R}_{i}(n)$ is the average data rate of user $i$ in the past WIN time periods (note that user $i$ may use different channels of different BSs in different time periods). For user $i$, the value of $\frac{\hat{r}_{i}^{(k)}(n)}{R_{i}(n)}$ achieves the largest when it selects channel $k$ of $\mathrm{BS} j$ at time instant $n$ among all the BSs. BS $j$ will select the user with largest criterion value and notify the related user. Our simulation results show that this scheme can achieve a moderate fairness (if we use Jain's fairness index ([32]) for evaluation, our simulation shows that we can achieve Jain's fairness index value above 0.7). The definition of Jain's fairness index is as follows:

$$
J(\mathbf{x})=\frac{\left(\sum_{i=1}^{M} x_{i}\right)^{2}}{M \cdot \sum_{i=1}^{M} x_{i}{ }^{2}} .
$$

Here, $M$ is the number of users, $x_{i}$ represents the $i$-th user data rate.

Similarly, to prevent too many channel requests from all the users and consuming too many control channels, we need also to introduce a threshold $I_{t h}$ for controlling the number of channel requests. Based on above analysis, the proposed algorithm 
can be expressed as:

\section{Algorithm II for Channel Access at Terminals}

For every terminal $i(i=1,2, \cdots, M)$, CSI of channel $k(k=1,2, \cdots, L)$ of $N$ BSs is estimated as $h_{i 1}^{(k)}, h_{i 2}^{(k)}, \cdots h_{i N}^{(k)}$, average data rate of terminal $i$ in the past WIN time periods is calculated as $\bar{R}_{i}(n)$.

If $\max \left(h_{i l}^{(k)}\right)=h_{i j}^{(k)},(l=1,2, \cdots, N)$

Then (1) Calculate the criterion value $i_{j}^{(k)}(n)=\frac{\hat{r}_{i j}^{(k)}(n)}{\hat{R}_{i}(n)}$,

$$
\hat{r}_{i j}^{(k)}(n)=\frac{W}{L} \cdot \log \left(1+\frac{P h_{i j}^{(k)}}{N_{0} \cdot \frac{W}{L}+P \sum_{l \neq j} h_{i l}^{(k)}}\right)
$$

(2) If $i_{j}^{(k)}(n) \geq I_{t h}$, send Channel Reserve Request to BS $j$

(3) Wait for notification, if got, transmit using channel $k$

Else Wait for next Coherence Time, check new estimated channel info. and calculate again.

\subsection{Summary}

In this chapter, we briefly discussed our system model. Based on the research proposal discussed in chapter 2, we proposed a distributed scheduling framework and a possible implementation method. This framework can be used for different scheduling purposes. We also proposed two algorithms for the goals of sum throughput maximization and proportional fairness approximation. This built a good foundation for the mathematical analysis in the next chapter. 


\section{Chapter 4}

\section{Mathematical Analysis}

To facilitate the analysis, we summarize the notations as in Table 4.1. First, we use homogeneous channel model for mathematical analysis. Here, "homogeneous" means that the probability density function of all the channels is same. After that, we will consider "heterogeneous" model which means that the probability density function of channels is different from channel to channel. Later, we will see some of the conclusions are similar for both homogeneous and heterogeneous channel models.

\subsection{Homogeneous Channel Model}

Based on order statistics [33], assuming each channel power gain is i.i.d, the joint distribution of $N$ channel power gains is

$$
f\left(h_{1}, h_{2}, \ldots, h_{N}\right)=N \cdot \prod_{i=1}^{N} f\left(h_{i}\right), \quad\left(h_{1} \geq h_{2}, \ldots, h_{N}\right) .
$$

So the probability that user $i$ attempt to reserve channel $k$ of BS $j$ (we use $j^{(k)}$ 
Table 4.1: Summary of notations.

\begin{tabular}{c|l}
\hline \hline Symbols & Meanings of Symbols \\
\hline \hline$N$ & The number of BSs \\
\hline$L$ & The number of channels in every BS \\
\hline$M$ & The number of users \\
\hline$H_{t h}$ & Threshold of channel power gain \\
\hline$\alpha$ & Attenuation factor \\
\hline$j^{(k)}$ & Channel $k$ of BS $j$ \\
\hline$h_{i j}^{(k)}$ & Channel power gain of user $i$ for channel $k$ of BS $j$ \\
\hline$f(h)$ & Probability density function of channel power gain \\
\hline$F(h)$ & Cumulative distribution function of channel power gain \\
\hline$P_{r}(\varepsilon)$ & Probability of event $\varepsilon$ \\
\hline$p_{a}$ & $P_{r}\left(\right.$ user $i$ attempts to reserve $\left.j^{(k)}\right)$ \\
\hline$p_{t}$ & $P_{r}\left(\right.$ user $i$ transmits using $\left.j^{(k)}\right)$ \\
\hline$P_{i j}^{(k)}$ & Power used by user $i$ in the channel $k$ of BS $j$ \\
\hline $\bar{R}_{i j}^{(k)}$ & Average data rate for user $i$ in the channel $k$ of BS $j$ \\
\hline$\gamma(h)$ & SINR function of channel power gain $h$ \\
\hline $\bar{T}_{i j}^{(k)}$ & The average throughput for user $i$ in the channel $k$ of BS $j$ \\
\hline$\tilde{h}_{i j}^{(k)}$ & Channel power gain from the user of channel $k$ of $i$-th BS to the $j$-th BS \\
\hline & \\
\hline & \\
\hline
\end{tabular}


to represent channel $k$ of BS $j$ to save space) can be calculated as

$$
\begin{aligned}
& P_{r}\left(\text { user } i \text { attempts to reserve } j^{(k)}\right) \\
= & \frac{1}{N} \cdot N \cdot \int_{H_{t h}}^{\infty} f\left(h_{1}\right) \mathrm{d} h_{1} \iint \cdots \int_{0}^{\alpha H_{t h}} \prod_{i=2}^{N} f\left(h_{i}\right) \mathrm{d} h_{2} \mathrm{~d} h_{3} \cdots \mathrm{d} h_{N} \\
= & {\left[1-F\left(H_{t h}\right)\right] \cdot F^{N-1}\left(\alpha H_{t h}\right) } \\
= & p_{a} \quad(0 \leq \alpha \leq 1) .
\end{aligned}
$$

Here, $\frac{1}{N}$ is the probability that channel power gain of channel $k$ of $\mathrm{BS} j$ is the greatest one among $N$ values.

The probability that user $i$ can actually get channel $k$ of BS $j$ can be calculated as

$$
\begin{aligned}
& P_{r}\left(\text { user } i \text { transmits using } j^{(k)}\right) \\
= & \sum_{l=1}^{M} P_{r}\left(\text { user } i \text { transmits using } j^{(k)}, l \text { users contend }\right) \\
= & \sum_{l=1}^{M} \frac{1}{l} \cdot\left(\begin{array}{c}
M-1 \\
l-1
\end{array}\right) \cdot p_{a}^{l} \cdot\left(1-p_{a}\right)^{M-l} \\
= & \frac{1}{M} \cdot\left[1-\left(1-p_{a}\right)^{M}\right] \\
= & p_{t} .
\end{aligned}
$$

If there is an average power constraint $P_{a v}$, we have

$$
\sum_{j, k} \mathbf{E}\left(P_{i j}^{(k)}\right) \leq P_{a v}
$$


If there is a peak power constraint $P_{\max }$, we have

$$
\max \left(\sum_{j, k} P_{i j}^{(k)}\right) \leq P_{\max }
$$

Next, we analyze the average power used by a single user when transmitting in this multi-channel system. As stipulated by our system model, a single user can occupy at most $L$ channels. We use $P_{i}$ to represent power level used in every channel when a single user transmits in $i$ channels. So the average power $\bar{P}$ per channel is

$$
\bar{P}=\sum_{i=1}^{L}\left(\begin{array}{c}
L \\
i
\end{array}\right) p_{t}^{i}\left(1-p_{t}\right)^{L-i} P_{i}
$$

The ratio of the $i$-th item to the first item of the polynomial (4.6) is

$$
\begin{aligned}
& \frac{\left(\begin{array}{l}
L \\
i
\end{array}\right) p_{t}{ }^{i}\left(1-p_{t}\right)^{L-i} P_{i}}{\left(\begin{array}{c}
L \\
1
\end{array}\right) p_{t}\left(1-p_{t}\right)^{L-1} P_{1}} \\
= & \frac{\left(\begin{array}{l}
L \\
i
\end{array}\right) P_{i}}{\left(\begin{array}{l}
L \\
1
\end{array}\right) P_{1}} \cdot\left(\frac{p_{t}}{1-p_{t}}\right)^{i-1}, \quad(2 \leq i \leq L) .
\end{aligned}
$$

The first part of (4.7) is obviously bounded by a certain value given a fixed $L$, and the second part converges to zero when $M$ goes to infinity. This leads to

$$
\lim _{M \rightarrow \infty} \frac{\left(\begin{array}{c}
L \\
i
\end{array}\right) P_{i}}{\left(\begin{array}{l}
L \\
1
\end{array}\right) P_{1}} \cdot\left(\frac{p_{t}}{1-p_{t}}\right)^{i-1}=0, \quad(2 \leq i \leq L) .
$$

The result of (4.8) implies that we can ignore the possibility of a single user transmitting more than one channel at the same time assuming the number of users $M$ is considerably large. This is a good approximation in our following analysis. In this way, (4.4) and (4.5) can be further reduced as

$$
\mathbf{E}\left(P_{i j}^{(k)}\right) \leq P_{a v}
$$




$$
\max \left(P_{i j}^{(k)}\right) \leq P_{\max }
$$

We use Shannon Equation to approximate the instantaneous data rate as

$$
R(\gamma(h))=\frac{W}{L} \cdot \log (1+\gamma(h))
$$

Refer to the channel power gain matrix of channel $k$ as follows, it can be easily found that the underlined entry in $j$-th column is the channel power gain of channel $k$ user of $j$-th BS (i.e., same value as $h_{i j}^{(k)}$ ) and other entries in this column are the channel power gains of all co-channel interferers (all other BSs' channel $k$ users):

$$
\left(\begin{array}{ccc|c|cc}
\tilde{h}_{11}^{(k)} & \tilde{h}_{12}^{(k)} & \ldots & \tilde{h}_{1 j}^{(k)} & \ldots & \tilde{h}_{1 N}^{(k)} \\
\tilde{h}_{21}^{(k)} & \tilde{h}_{22}^{(k)} & \ldots & \tilde{h}_{2 j}^{(k)} & \ldots & \tilde{h}_{2 N}^{(k)} \\
\vdots & \vdots & \ddots & \vdots & \ddots & \vdots \\
\tilde{h}_{j 1}^{(k)} & \tilde{h}_{j 2}^{(k)} & \ldots & \tilde{h}_{j j}^{(k)} & \ldots & \tilde{h}_{2 N}^{(k)} \\
\vdots & \vdots & \ddots & \vdots & \ddots & \vdots \\
\tilde{h}_{N 1}^{(k)} & \tilde{h}_{N 2}^{(k)} & \ldots & \tilde{h}_{N j}^{(k)} & \ldots & \tilde{h}_{N N}^{(k)}
\end{array}\right)
$$

Assuming ergodicity of all users and channels, the joint probability density function is

$$
\begin{aligned}
f\left(\tilde{h}_{j j}^{(k)}, \tilde{h}_{1 j}^{(k)}, \tilde{h}_{2 j}^{(k)}, \ldots, \tilde{h}_{N j}^{(k)}\right)= & N \cdot f\left(\tilde{h}_{j j}^{(k)}\right) \cdot \prod_{\substack{i=1 \\
i \neq j}}^{N} f\left(\tilde{h}_{i j}^{(k)}\right), \\
& \left(\tilde{h}_{j j}^{(k)} \geq \tilde{h}_{1 j}^{(k)}, \tilde{h}_{2 j}^{(k)}, \ldots, \tilde{h}_{j-1, j}^{(k)}, \tilde{h}_{j+1, j}^{(k)}, \ldots, \tilde{h}_{N j}^{(k)}\right) .
\end{aligned}
$$

If user $i$ transmits in channel $k$ of BS $j$, the related Signal-to-Interference-NoiseRatio (SINR) can be represented as (assuming $N_{0}$ as the single band power spectral 
density of gaussian noise at receivers)

$$
\gamma\left(h_{i j}^{(k)}, h_{1 j}^{(k)}, h_{2 j}^{(k)}, \ldots, h_{N j}^{(k)}\right)=\frac{\tilde{h}_{j j}^{(k)} \cdot P\left(\tilde{h}_{j j}^{(k)}\right)}{N_{0} \cdot \frac{W}{L}+\sum_{l \neq j} \tilde{h}_{l j}^{(k)} \cdot P\left(\tilde{h}_{l l}^{(k)}\right)} .
$$

Average data rate for user $i$ on the channel $k$ of $\mathrm{BS} j$ is

$\bar{R}_{i j}^{(k)}=\mathbf{E}\left(R\left(\gamma\left(h_{1}, h_{2}, \cdots, h_{N}\right)\right) \mid\right.$ user $i$ transmits using $\left.j^{(k)}\right)$

$$
\begin{aligned}
& =\int_{H_{t h}}^{\infty} \iint \cdots \int_{0}^{\alpha H_{t h}} R\left(\gamma\left(h_{1}, h_{2}, \cdots, h_{N}\right)\right) . \\
& \quad P_{r}\left(\tilde{h}_{j j}^{(k)}=h_{1}, \tilde{h}_{1 j}^{(k)}=h_{2}, \cdots \tilde{h}_{N j}^{(k)}=h_{N} \mid \text { user } i \text { transmits using } j^{(k)}\right) \mathrm{d} h_{1} \cdots \mathrm{d} h_{N} .
\end{aligned}
$$

Note that

$$
\begin{aligned}
& P_{r}\left(\tilde{h}_{j j}^{(k)}=h_{1}, \tilde{h}_{1 j}^{(k)}=h_{2}, \cdots, \tilde{h}_{N j}^{(k)}=h_{N} \mid \text { user } i \text { transmits using } j^{(k)}\right) \\
= & \frac{P_{r}\left(\tilde{h}_{j j}^{(k)}=h_{1}, \cdots, \tilde{h}_{N j}^{(k)}=h_{N}\right) \cdot P_{r}\left(\text { user } i \text { transmits using } j^{(k)} \mid \tilde{h}_{j j}^{(k)}=h_{1}, \cdots, \tilde{h}_{N j}^{(k)}=h_{N}\right)}{P_{r}\left(\text { user } i \text { transmits using } j^{(k)}\right)} \\
= & \frac{\frac{1}{N} \cdot N \cdot \prod_{i=1}^{N} f\left(h_{i}\right) \cdot \frac{\frac{1}{M} \cdot\left[1-\left(1-p_{a}\right)^{M}\right]}{\left[1-F\left(H_{t h}\right)\right] \cdot F^{N-1}\left(\alpha H_{t h}\right)}}{\frac{1}{M} \cdot\left[1-\left(1-p_{a}\right)^{M}\right]} \\
= & \frac{\prod_{i=1}^{N} f\left(h_{i}\right)}{\left[1-F\left(H_{t h}\right)\right] \cdot F^{N-1}\left(\alpha H_{t h}\right)} .
\end{aligned}
$$

Considering the result we get from (4.15), we can get

$$
\bar{R}_{i j}^{(k)}=\frac{\int_{H_{t h}}^{\infty} \iint \cdots \int_{0}^{\alpha H_{t h}} R\left(\gamma\left(h_{1}, h_{2}, \cdots, h_{N}\right)\right) \cdot \prod_{i=1}^{N} f\left(h_{i}\right) \mathrm{d} h_{1} \cdots \mathrm{d} h_{N}}{\left[1-F\left(H_{t h}\right)\right] \cdot F^{N-1}\left(\alpha H_{t h}\right)} .
$$


Probabilistically, the average throughput of user $i$ in channel $k$ of $j$-th BS is

$$
\bar{T}_{i j}^{(k)}=P_{r}\left(\text { user } i \text { transmits using } j^{(k)}\right) \cdot \bar{R}_{i j}^{(k)} .
$$

The total average throughput of $M$ users in $N$ BSs with $L$ channels each is

$$
\begin{aligned}
\sum_{i, j, k} \bar{T}_{i j}^{(k)} & =\sum_{i, j, k} P_{r}\left(\text { user } i \text { transmits using } j^{(k)}\right) \cdot \bar{R}_{i j}^{(k)} \\
& =\sum_{i, j, k} \frac{1}{M} \cdot\left[1-\left(1-p_{a}\right)^{M}\right] \cdot \bar{R}_{i j}^{(k)}
\end{aligned}
$$

subject to two constraints as

$$
\text { s.t. }\left\{\begin{array}{l}
\mathbf{E}\left(P_{i j}^{(k)}\right) \leq P_{a v}, \\
\max \left(P_{i j}^{(k)}\right) \leq P_{\max }
\end{array}\right.
$$

Assuming identical distribution of all the users and channels, (4.18) can be further represented as

$$
\begin{aligned}
\sum_{i, j, k} \bar{T}_{i j}^{(k)} & =M \cdot N \cdot L \cdot \frac{1}{M} \cdot\left[1-\left(1-p_{a}\right)^{M}\right] \cdot \bar{R}_{i j}^{(k)} \\
& =\left[1-\left(1-p_{a}\right)^{M}\right] \cdot N \cdot L \cdot \bar{R}_{i j}^{(k)}
\end{aligned}
$$

\subsubsection{Power Allocation with Channel Inversion}

First, assume power allocation using channel inversion, which means that once the user transmits with channel power gain $h$, the transmit power used is directly given by $\frac{P_{\text {recv }}}{h}$, where $P_{\text {recv }}$ is the equal received power level at every BS. Note that we assume that the number of users is considerably large, so we can always select "best" channels which ensure that the power allocation at terminals is feasible. 
Then, (4.16) can be represented as

$$
\begin{aligned}
& \bar{R}_{i j}^{(k)}=\frac{\int_{H_{t h}}^{\infty} \iint \cdots \int_{0}^{\alpha H_{t h}} R\left(\gamma\left(h_{1}, h_{2}, \cdots, h_{N}\right)\right) \cdot \prod_{i=1}^{N} f\left(h_{i}\right) \mathrm{d} h_{1} \cdots \mathrm{d} h_{N}}{\left[1-F\left(H_{t h}\right)\right] \cdot F^{N-1}\left(\alpha H_{t h}\right)} \\
& =\frac{\int_{H_{t h}}^{\infty} \iint \cdots \int_{0}^{\alpha H_{t h}} \frac{W}{L} \cdot \log \left(1+\frac{\tilde{h}_{j j}^{(k)} \cdot P\left(\tilde{h}_{j j}^{(k)}\right)}{N_{0} \cdot \frac{W}{L}+\sum_{l \neq j} \tilde{h}_{l j}^{(k)} \cdot P\left(\tilde{h}_{l l}^{(k)}\right)}\right) \cdot \prod_{i=1}^{N} f\left(h_{i}\right) \mathrm{d} h_{1} \cdots \mathrm{d} h_{N}}{\left[1-F\left(H_{t h}\right)\right] \cdot F^{N-1}\left(\alpha H_{t h}\right)} \\
& =\frac{W}{L} \cdot \frac{\int_{H_{t h}}^{\infty} \iint \cdots \int_{0}^{\alpha H_{t h}} \log \left(1+\frac{P_{\text {recv }}}{N_{0} \cdot \frac{W}{L}+\sum_{l \neq j} \tilde{h}_{l j}^{(k)} \cdot \frac{P_{r e c v}}{\tilde{h}_{l l}^{(k)}}}\right) \cdot \prod_{i=1}^{N} f\left(h_{i}\right) \mathrm{d} h_{1} \cdots \mathrm{d} h_{N}}{\left[1-F\left(H_{t h}\right)\right] \cdot F^{N-1}\left(\alpha H_{t h}\right)} \\
& =\frac{W}{L} \cdot \mathbf{E}\left[\log \left(1+\frac{P_{r e c v}}{N_{0} \cdot \frac{W}{L}+\sum_{l \neq j} \tilde{h}_{l j}^{(k)} \cdot \frac{P_{r e c v}}{\tilde{h}_{l l}^{(k)}}}\right)\right] \\
& \geq \frac{W}{L} \cdot \log \left(1+\frac{P_{r e c v}}{N_{0} \cdot \frac{W}{L}+\sum_{l \neq j} \frac{\alpha H_{t h}}{H_{t h}} \cdot P_{\text {recv }}}\right) \cdot \frac{\int_{H_{t h}}^{\infty} \iint \cdots \int_{0}^{\alpha H_{t h}} \cdot \prod_{i=1}^{N} f\left(h_{i}\right) \mathrm{d} h_{1} \cdots \mathrm{d} h_{N}}{\left[1-F\left(H_{t h}\right)\right] \cdot F^{N-1}\left(\alpha H_{t h}\right)} \\
& =\frac{W}{L} \cdot \log \left[1+\frac{P_{\text {recv }}}{N_{0} \cdot \frac{W}{L}+\alpha \cdot(N-1) P_{\text {recv }}}\right] \text {. } \\
& \sum_{i, j, k} \bar{T}_{i j}^{(k)}=\left[1-\left(1-p_{a}\right)^{M}\right] \cdot N \cdot L \cdot \bar{R}_{i j}^{(k)} \\
& \geq\left[1-\left(1-p_{a}\right)^{M}\right] \cdot N \cdot L \cdot \frac{W}{L} \cdot \log \left[1+\frac{P_{\text {recv }}}{N_{0} \cdot \frac{W}{L}+\alpha \cdot(N-1) P_{\text {recv }}}\right] \\
& =\left[1-\left(1-p_{a}\right)^{M}\right] \cdot N \cdot W \cdot \log \left[1+\frac{P_{\text {recv }}}{N_{0} \cdot \frac{W}{L}+\alpha(N-1) \cdot P_{\text {recv }}}\right] \text {. }
\end{aligned}
$$

It can be seen that the total average throughput is lower bounded by (4.22) for all the values of $\alpha$. Asymptotically, the first part of (4.22) equals one as the 
number of users goes to infinity and this is obviously the result of exploiting multiuser diversity. The second part is $N$ times of the throughput of a single BS except that there is some co-channel interference caused by the users transmitting using the same channel of different BSs. If we can select those users who have good channel state information while causing little co-channel interference to other users then we can achieve more throughput while using the same spectrum width by way of spatial diversity (Multi-BS). The less the value of $\alpha$, the less co-channel interference to other users.

Compared with the scenario of centralized scheduling in a single BS with same bandwidth $W$ and number of channels $L$, where the total average throughput is

$$
\sum_{i, k} \bar{T}_{i 1}^{(k)}=W \cdot \log \left(1+\frac{P_{\text {recv }}}{N_{0} \cdot \frac{W}{L}}\right) .
$$

We have got the probability that user $i$ attempts to reserve channel $k$ of $\mathrm{BS} j$ as $p_{a}$ from (4.2). To make full use of all the channels in the system, the value of $p_{a}$ needs to be equal or greater than $\frac{1}{M}$, otherwise chances are that some channels will be wasted since that probabilistically the number of users attempting to reserve a certain channel is $M p_{a}$, the value of which should be equal to or greater than one.

$$
\begin{aligned}
& M \cdot p_{a}=M \cdot\left[1-F\left(H_{t h}\right)\right] \cdot F^{N-1}\left(\alpha H_{t h}\right) \geq 1 \\
\Longrightarrow & p_{a}=\left[1-F\left(H_{t h}\right)\right] \cdot F^{N-1}\left(\alpha H_{t h}\right) \geq \frac{1}{M} .
\end{aligned}
$$

If we fix the values of $H_{t h}, N$, it can be easily found that the minimum value of $\alpha$ is related to the values of $H_{t h}, N$, and $M$. This can be represented by $\alpha_{m i n}=$ $\psi\left(H_{t h}, M, N\right)$. It is obviously that $\alpha_{\min }$ is a monotonically decreasing function of $M$ 
and converges to $0^{+}$when $M$ goes to infinity, which can be formulated as

$$
\lim _{M \rightarrow \infty} \alpha_{\min }=\lim _{M \rightarrow \infty} \psi\left(H_{t h}, M, N\right)=0^{+}
$$

Based on the above analysis, we can get

$$
\begin{aligned}
\lim _{M \rightarrow \infty} \frac{\sum_{i, j, k} \bar{T}_{i j}^{(k)}}{\sum_{i, k} \bar{T}_{i 1}^{(k)}} & \geq \frac{\lim _{M \rightarrow \infty}\left[1-\left(1-p_{a}\right)^{M}\right] \cdot N \cdot W \cdot \log \left[1+\frac{P_{\text {recv }}}{N_{0} \cdot \frac{W}{L}+\alpha_{\text {min }}(N-1) \cdot P_{\text {recv }}}\right]}{W \cdot \log \left(1+\frac{P_{\text {recv }}}{N_{0} \cdot \frac{W}{L}}\right)} \\
& =N .
\end{aligned}
$$

From $(4.25), \alpha_{\min }$ approaches zero as close as possible as the number of users goes to infinity and this naturally leads to the result of (4.26). On the other hand, we have obviously the following inequality which means that the total average throughput can not be greater than $N$ times of the total average throughput of a single BS with centralized scheduling:

$$
\begin{aligned}
\sum_{i, j, k} \bar{T}_{i j}^{(k)} \leq N \cdot W \cdot \log \left(1+\frac{P_{\text {recv }}}{N_{0} \cdot \frac{W}{L}}\right) \\
\lim _{M \rightarrow \infty} \frac{\sum_{i, j, k} \bar{T}_{i j}^{(k)}}{\sum_{i, k} \bar{T}_{i 1}^{(k)}} \leq \lim _{M \rightarrow \infty} \frac{N \cdot W \cdot \log \left(1+\frac{P_{\text {recv }}}{N_{0} \cdot \frac{W}{L}}\right)}{W \cdot \log \left(1+\frac{P_{\text {recv }}}{N_{0} \cdot \frac{W}{L}}\right)} \\
=N .
\end{aligned}
$$


Combining (4.26) and (4.28), we can get

$$
\lim _{M \rightarrow \infty} \frac{\sum_{i, j, k} \bar{T}_{i j}^{(k)}}{\sum_{i, k} \bar{T}_{i 1}^{(k)}}=N .
$$

This means that asymptotically we can get $N$ times of the total average throughput of a single BS with centralized scheduling by fully exploiting multi-user diversity. Next, we will discuss how to select channel power gain threshold $H_{t h}$ and $\alpha$ to op-

timize the total average throughput $\sum_{i, j, k} \bar{T}_{i j}^{(k)}$ given that the number of users $M$ and BSs $N$ is known.

\subsubsection{Throughput Optimization with Moderate Population}

This means that the number of users located within the system is comparable with the number of channels, which can be represented as:

$$
M \sim N L
$$

In this case, it is better to use centralized scheduling to allocate power and channels to users. This is beyond the scope of this paper.

\subsubsection{Throughput Optimization with Dense Population}

In this scenario, the number of users in the system is much greater than the number of channels and this means that multi-user diversity can play a big role in 
throughput optimization. This requires that

$$
M \gg N L \text {. }
$$

Based on the result we get from (4.21a), we can see that the total average throughput is

$$
\sum_{i, j, k} \bar{T}_{i j}^{(k)}=\left[1-\left(1-p_{a}\right)^{M}\right] \cdot N \cdot W \cdot \mathbf{E}\left[\log \left(1+\frac{P_{\text {recv }}}{N_{0} \cdot \frac{W}{L}+\sum_{l \neq j} \tilde{h}_{l j}^{(k)} \cdot \frac{P_{\text {recv }}}{\tilde{h}_{l l}^{(k)}}}\right)\right] .
$$

The first part $\left[1-\left(1-p_{a}\right)^{M}\right]$ can be optimized by appropriately selecting the values of channel power gain threshold $H_{t h}$ and $\alpha$, while the last part $\mathbf{E}\left[\log \left(1+\frac{P_{\text {recv }}}{N_{0} \cdot \frac{W}{L}+\sum_{l \neq j} \tilde{h}_{l j}^{(k)} \cdot \frac{P_{r e c v}}{\tilde{h}_{l l}^{k(k)}}}\right)\right]$ is hard to optimize by the user itself since the value of channel power gains $\tilde{h}_{l j}^{(k)}$ and $\tilde{h}_{l l}^{(k)}$ can only be known by centralized scheduling (i.e. users can only get them from BSs). But we know that the total average throughput is lower bounded by $\left[1-\left(1-p_{a}\right)^{M}\right] \cdot N \cdot W \cdot \log \left(1+\frac{P_{\text {recv }}}{N_{0} \cdot \frac{W}{L}+\alpha(N-1) \cdot P_{\text {rec }}}\right)$, this motivates us to optimize this lower bound and we have proved that this lower bound is asymptotically optimal by (4.26), (4.28) and (4.29). This suboptimal method will guarantee that the total average throughput is at least greater than the optimized value of the lower bound. We can now formulate the following optimization problem:

$$
\begin{aligned}
\underset{\left(\text { over } \alpha, H_{t h}\right)}{\operatorname{Maximize}} & \left\{\left[1-\left(1-p_{a}\right)^{M}\right] \cdot N W \cdot \log \left[1+\frac{P_{\text {recv }}}{N_{0} \cdot \frac{W}{L}+\alpha(N-1) \cdot P_{\text {recv }}}\right]\right\} \\
\text { Subject to: } & p_{a}=\left[1-F\left(H_{t h}\right)\right] \cdot F^{N-1}\left(\alpha H_{t h}\right) \quad\left(0 \leq \alpha \leq 1,0 \leq H_{t h} \leq \infty\right) \\
& p_{a} \geq \frac{1}{M} .
\end{aligned}
$$

We can easily find local maximum values of the objective function in (4.33) using 
mature numerical methods. In this way, we get suboptimal values of $H_{t h}$ and $\alpha$. Note that if we can not find some sets that meet $p_{a} \geq \frac{1}{M}$, this should be categorized as the case of (4.31) and should be treated by centralized scheduling.

\subsubsection{Power Allocation with Interference Boundary}

Next, we give some analysis assuming that every user is interference bounded when allocating power, which means that the interference that it can cause to other users is upper bounded by $I$. So we have

$$
\begin{aligned}
& \max _{\substack{l=1,2, \cdots N \\
l \neq j}}\left[\tilde{h}_{l j}^{(k)} \cdot P\left(\tilde{h}_{l l}^{(k)}\right)\right]=I \\
\Longleftrightarrow & \alpha H_{t h} \cdot P\left(\tilde{h}_{l l}^{(k)}\right) \geq I \quad(l=1,2, \cdots, N) \\
\Longleftrightarrow & P\left(\tilde{h}_{l l}^{(k)}\right) \geq \frac{I}{\alpha H_{t h}} \quad(l=1,2, \cdots, N) .
\end{aligned}
$$

Considering (4.10) and (4.34) can be reformulated as

$$
\frac{I}{\alpha H_{t h}} \leq P\left(\tilde{h}_{l l}^{(k)}\right) \leq P_{\max }
$$

Based on above analysis, we have the following inequality for (4.13)

$$
\begin{aligned}
\frac{\tilde{h}_{j j}^{(k)} \cdot P\left(\tilde{h}_{j j}^{(k)}\right)}{N_{0} \cdot \frac{W}{L}+\sum_{l \neq j} \tilde{h}_{l j}^{(k)} \cdot P\left(\tilde{h}_{l l}^{(k)}\right)} & \geq \frac{H_{t h} \cdot \frac{I}{\alpha H_{t h}}}{N_{0} \cdot \frac{W}{L}+\sum_{l \neq j} I} \\
& =\frac{\frac{I}{\alpha}}{N_{0} \cdot \frac{W}{L}+(N-1) I}
\end{aligned}
$$


Then, (4.16) can be represented as

$$
\begin{aligned}
\bar{R}_{i j}^{(k)} & =\frac{\int_{H_{t h}}^{\infty} \iint \cdots \int_{0}^{\alpha H_{t h}} R\left(\gamma\left(h_{1}, h_{2}, \cdots, h_{N}\right)\right) \cdot \prod_{i=1}^{N} f\left(h_{i}\right) \mathrm{d} h_{1} \cdots \mathrm{d} h_{N}}{\left[1-F\left(H_{t h}\right)\right] \cdot F^{N-1}\left(\alpha H_{t h}\right)} \\
& =\frac{W}{L} \cdot \frac{\int_{H_{t h}}^{\infty} \iint \cdots \int_{0}^{\alpha H_{t h}} \log \left(1+\frac{\tilde{h}_{j j}^{(k)} \cdot P\left(\tilde{h}_{j j}^{(k)}\right)}{N_{0} \cdot \frac{W}{L}+\sum_{l \neq j}^{(k)} \tilde{h}_{l j}^{(k)} \cdot P\left(\tilde{h}_{l l}^{(k)}\right)}\right) \cdot \prod_{i=1}^{N} f\left(h_{i}\right) \mathrm{d} h_{1} \cdots \mathrm{d} h_{N}}{\left[1-F\left(H_{t h}\right)\right] \cdot F^{N-1}\left(\alpha H_{t h}\right)} \\
& \geq \frac{W}{L} \cdot \frac{\int_{H_{t h}}^{\infty} \iint \cdots \int_{0}^{\alpha H_{t h}} \log \left[1+\frac{\frac{I}{\alpha}}{N_{0} \cdot \frac{W}{L}+(N-1) I}\right] \cdot \prod_{i=1}^{N} f\left(h_{i}\right) \mathrm{d} h_{1} \cdots \mathrm{d} h_{N}}{\left[1-F\left(H_{t h}\right)\right] \cdot F^{N-1}\left(\alpha H_{t h}\right)} \\
& =\frac{W}{L} \cdot \log \left[1+\frac{\frac{I}{\alpha}}{N_{0} \cdot \frac{W}{L}+(N-1) I}\right]
\end{aligned}
$$

The total average throughput is reformulated as

$$
\begin{aligned}
\sum_{i, j, k} \bar{T}_{i j}^{(k)} & =\left[1-\left(1-p_{a}\right)^{M}\right] \cdot N \cdot L \cdot \bar{R}_{i j}^{(k)} \\
& \geq\left[1-\left(1-p_{a}\right)^{M}\right] \cdot N \cdot L \cdot \frac{W}{L} \cdot \log \left[1+\frac{\frac{I}{\alpha}}{N_{0} \cdot \frac{W}{L}+(N-1) I}\right] \\
& =\left[1-\left(1-p_{a}\right)^{M}\right] \cdot N \cdot W \cdot \log \left[1+\frac{\frac{I}{\alpha}}{N_{0} \cdot \frac{W}{L}+(N-1) I}\right]
\end{aligned}
$$

Similarly, we can now formulate the following optimization problem

$$
\begin{aligned}
& \underset{\left(\text { over } \alpha, I, H_{t h}\right)}{\operatorname{Maximize}}\left\{\left[1-\left(1-p_{a}\right)^{M}\right] \cdot N W \cdot \log \left[1+\frac{\frac{I}{\alpha}}{N_{0} \cdot \frac{W}{L}+(N-1) I}\right]\right\} \\
& \text { Subject to: } p_{a}=\left[1-F\left(H_{t h}\right)\right] \cdot F^{N-1}\left(\alpha H_{t h}\right) \\
& p_{a} \geq \frac{1}{M} \\
& \frac{I}{\alpha H_{t h}} \leq P_{\text {max }}, \quad\left(0 \leq \alpha \leq 1,0 \leq H_{t h} \leq \infty\right) .
\end{aligned}
$$




\subsubsection{Power Allocation With Equality}

In this case, all users use the same power level $P$ for transmission, then we have the following inequality for (4.13)

$$
\begin{aligned}
\frac{\tilde{h}_{j j}^{(k)} \cdot P\left(\tilde{h}_{j j}^{(k)}\right)}{N_{0} \cdot \frac{W}{L}+\sum_{l \neq j} \tilde{h}_{l j}^{(k)} \cdot P\left(\tilde{h}_{l l}^{(k)}\right)} & \geq \frac{H_{t h} \cdot P}{N_{0} \cdot \frac{W}{L}+\sum_{l \neq j} \alpha H_{t h} P} \\
& =\frac{H_{t h} \cdot P}{N_{0} \cdot \frac{W}{L}+(N-1) \alpha H_{t h} P}
\end{aligned}
$$

Then, (4.16) can be represented as

$$
\begin{aligned}
\bar{R}_{i j}^{(k)} & =\frac{\int_{H_{t h}}^{\infty} \iint \cdots \int_{0}^{\alpha H_{t h}} R\left(\gamma\left(h_{1}, h_{2}, \cdots, h_{N}\right)\right) \cdot \prod_{i=1}^{N} f\left(h_{i}\right) \mathrm{d} h_{1} \cdots \mathrm{d} h_{N}}{\left[1-F\left(H_{t h}\right)\right] \cdot F^{N-1}\left(\alpha H_{t h}\right)} \\
& =\frac{W}{L} \cdot \frac{\int_{H_{t h}}^{\infty} \iint \cdots \int_{0}^{\alpha H_{t h}} \log \left(1+\frac{\tilde{h}_{j j}^{(k)} \cdot P\left(\tilde{h}_{j j}^{(k)}\right)}{N_{0} \cdot \frac{W}{L}+\sum_{l \neq j} \tilde{h}_{l j}^{(k)} \cdot P\left(\tilde{h}_{l l}^{(k)}\right)}\right) \cdot \prod_{i=1}^{N} f\left(h_{i}\right) \mathrm{d} h_{1} \cdots \mathrm{d} h_{N}}{\left[1-F\left(H_{t h}\right)\right] \cdot F^{N-1}\left(\alpha H_{t h}\right)} \\
& \geq \frac{W}{L} \cdot \frac{\int_{H_{t h}}^{\infty} \iint \cdots \int_{0}^{\alpha H_{t h}} \log \left[1+\frac{H_{t h} \cdot P}{N_{0} \cdot \frac{W}{L}+(N-1) \alpha H_{t h} P}\right] \cdot \prod_{i=1}^{N} f\left(h_{i}\right) \mathrm{d} h_{1} \cdots \mathrm{d} h_{N}}{\left[1-F\left(H_{t h}\right)\right] \cdot F^{N-1}\left(\alpha H_{t h}\right)} \\
& =\frac{W}{L} \cdot \log \left[1+\frac{H_{t h} \cdot P}{N_{0} \cdot \frac{W}{L}+(N-1) \alpha H_{t h} P}\right]
\end{aligned}
$$

The total average throughput is reformulated as

$$
\begin{aligned}
\sum_{i, j, k} \bar{T}_{i j}^{(k)} & =\left[1-\left(1-p_{a}\right)^{M}\right] \cdot N \cdot L \cdot \bar{R}_{i j}^{(k)} \\
& \geq\left[1-\left(1-p_{a}\right)^{M}\right] \cdot N \cdot L \cdot \frac{W}{L} \cdot \log \left[1+\frac{H_{t h} \cdot P}{N_{0} \cdot \frac{W}{L}+(N-1) \alpha H_{t h} P}\right] \\
& =\left[1-\left(1-p_{a}\right)^{M}\right] \cdot N \cdot W \cdot \log \left[1+\frac{H_{t h} \cdot P}{N_{0} \cdot \frac{W}{L}+(N-1) \alpha H_{t h} P}\right]
\end{aligned}
$$

Similarly, we can also get the result of (4.29). We can get this conclusion by 
thinking this way: whenever a BS in centralized scheduling chooses a user with a "good" channel $h$, we can almost always find in our system model a user also with a "good" channel $h$ and little interference to other users if the number of users goes infinity. In this way, we can easily get the conclusion of (4.29).

For the optimization problem, we can now formulate as follows:

$$
\begin{aligned}
\underset{\left(\text { over } \alpha, P, H_{t h}\right)}{\operatorname{Maximize}} & \left\{\left[1-\left(1-p_{a}\right)^{M}\right] \cdot N W \cdot \log \left[1+\frac{H_{t h} \cdot P}{N_{0} \cdot \frac{W}{L}+(N-1) \alpha H_{t h} P}\right]\right\} \\
\text { Subject to: } & p_{a}=\left[1-F\left(H_{t h}\right)\right] \cdot F^{N-1}\left(\alpha H_{t h}\right) \quad\left(0 \leq \alpha \leq 1,0 \leq H_{t h} \leq \infty\right) \\
& p_{a} \geq \frac{1}{M} \\
P & \leq P_{\text {max }} .
\end{aligned}
$$

\subsection{Heterogeneous Channel Model}

Similarly, based on order statistics [33], assuming heterogeneous channel model, the joint distribution of $N$ channel power gains of terminal $i$ for channel $k$ is

$$
\begin{gathered}
f_{i}^{(k)}\left(h_{1}, h_{2}, \ldots, h_{N}\right)=\sum \underbrace{f_{i j_{1}}^{(k)}\left(h_{1}\right) f_{i j_{2}}^{(k)}\left(h_{2}\right) \cdots f_{i j_{N}}^{(k)}\left(h_{N}\right)}_{j_{1} j_{2} \ldots j_{N} \in\{1,2 \ldots N\}}, \\
\left(h_{1} \geq h_{2}, \ldots, h_{N}\right) .
\end{gathered}
$$

The probability that user $i$ will attempt to reserve channel $k$ of BS $j$ can be 
calculated as

$$
\begin{aligned}
& P_{r}\left(\text { user } i \text { attempts to reserve } j^{(k)}\right) \\
= & \int_{H_{t h}}^{\infty} f_{i j}^{(k)}\left(h_{1}\right) \mathrm{d} h_{1} \iint \cdots \int_{0}^{\alpha H_{t h}} f_{i j_{2}}^{(k)}\left(h_{2}\right) \ldots f_{i j_{N}}^{(k)}\left(h_{N}\right) \mathrm{d} h_{2} \mathrm{~d} h_{3} \cdots \mathrm{d} h_{N} \\
= & {\left[1-F_{i j}^{(k)}\left(H_{t h}\right)\right] \cdot \underbrace{F_{i j_{2}}^{(k)}\left(\alpha H_{t h}\right) F_{i j_{3}}^{(k)}\left(\alpha H_{t h}\right) \cdots F_{i j_{N}}^{(k)}\left(\alpha H_{t h}\right)}_{j_{2} j_{3} \cdots j_{N} \in\{\{1,2 \cdots N\}-j\}} } \\
= & p_{a, i j}^{(k)}, \quad(0 \leq \alpha \leq 1) .
\end{aligned}
$$

The probability that user $i$ can actually get channel $k$ of BS $j$ can be calculated as

$$
\begin{aligned}
& P_{r}\left(\text { user } i \text { transmits using } j^{(k)}\right) \\
= & \sum_{l=1}^{M} P_{r}\left(\text { user } i \text { transmits using } j^{(k)}, l \text { users contend }\right) \\
= & \sum_{l=1}^{M} \frac{1}{l} \cdot p_{a, i j}^{(k)} \cdot \sum \underbrace{p_{a, i^{2} j}^{(k)} \cdots p_{a, i^{l} j}^{(k)}}_{i^{2} i^{3} \cdots i^{M} \in\{\{1,2 \cdots M\}-i\}} \cdot \underbrace{[l-1) \text { items }}_{(M-l) \text { items }} \\
= & p_{t, i j}^{(k)} .
\end{aligned}
$$

Similarly, average data rate for user $i$ on the channel $k$ of BS $j$ is

$$
\begin{aligned}
\bar{R}_{i j}^{(k)}= & \mathbf{E}\left(R\left(\gamma\left(h_{1}, h_{2}, \cdots, h_{N}\right)\right) \mid \text { user } i \text { transmits using } j^{(k)}\right) \\
= & \int_{H_{t h}}^{\infty} \iint \cdots \int_{0}^{\alpha H_{t h}} R\left(\gamma\left(h_{1}, h_{2}, \cdots, h_{N}\right)\right) \cdot \\
& P_{r}\left(\tilde{h}_{j j}^{(k)}=h_{1}, \tilde{h}_{1 j}^{(k)}=h_{2}, \cdots \tilde{h}_{N j}^{(k)}=h_{N} \mid \text { user } i \text { transmits using } j^{(k)}\right) \mathrm{d} h_{1} \cdots \mathrm{d} h_{N} .
\end{aligned}
$$




$$
\begin{aligned}
& P_{r}\left(\tilde{h}_{j j}^{(k)}=h_{1}, \tilde{h}_{1 j}^{(k)}=h_{2}, \cdots, \tilde{h}_{N j}^{(k)}=h_{N} \mid \text { user } i \text { transmits using } j^{(k)}\right) \\
= & \frac{P_{r}\left(\tilde{h}_{j j}^{(k)}=h_{1}, \cdots, \tilde{h}_{N j}^{(k)}=h_{N}\right) \cdot P_{r}\left(\text { user } i \text { transmits using } j^{(k)} \mid \tilde{h}_{j j}^{(k)}=h_{1}, \cdots, \tilde{h}_{N j}^{(k)}=h_{N}\right)}{P_{r}\left(\text { user } i \text { transmits using } j^{(k)}\right)} \\
= & \frac{f_{i j}^{(k)}\left(h_{1}\right) f_{i j_{2}}^{(k)}\left(h_{2}\right) \cdots f_{i j_{N}}^{(k)}\left(h_{N}\right) \cdot \frac{p_{t, i j}}{\left[1-F_{i j}^{(k)}\left(H_{t h}\right)\right] \cdot F_{i j_{2}}^{(k)}\left(\alpha H_{t h}\right) F_{i j_{3}}^{(k)}\left(\alpha H_{t h}\right) \cdots F_{i j_{N}}^{(k)}\left(\alpha H_{t h}\right)}}{p_{t, i j}^{(k)}} \\
= & \underbrace{\frac{\left[1-F_{i j}^{(k)}\left(H_{t h}\right)\right] \cdot F_{i j_{2}}^{(k)}\left(\alpha H_{t h}\right) F_{i j_{3}}^{(k)}\left(\alpha H_{t h}\right) \cdots F_{i j_{N}}^{(k)}\left(\alpha H_{t h}\right)}{(k)}\left(h_{2}\right) \cdots f_{j_{N}}^{(k)}\left(h_{N}\right)}_{j_{2} \ldots j_{N} \in\{\{1,2 \ldots N\}-j\}} .
\end{aligned}
$$

Based on the results from above (4.48), we can get

$$
\bar{R}_{i j}^{(k)}=\underbrace{\frac{\int_{H_{t h}}^{\infty} \iint \cdots \int_{0}^{\alpha H_{t h}} R\left(\gamma\left(h_{1}, h_{2}, \cdots, h_{N}\right)\right) \cdot f_{i j}^{(k)}\left(h_{1}\right) f_{i j_{2}}^{(k)}\left(h_{2}\right) \cdots f_{i j_{N}}^{(k)}\left(h_{N}\right) \mathrm{d} h_{1} \cdots \mathrm{d} h_{N}}{\left[1-F_{i j}^{(k)}\left(H_{t h}\right)\right] \cdot F_{i j_{2}}^{(k)}\left(\alpha H_{t h}\right) F_{i j_{3}}^{(k)}\left(\alpha H_{t h}\right) \cdots F_{i j_{N}}^{(k)}\left(\alpha H_{t h}\right)}}_{j_{2} \ldots j_{N} \in\{\{1,2 \ldots N\}-j\}} .
$$

The total average throughput of $M$ users in $N$ BSs with $L$ channels each is

$$
\begin{aligned}
\sum_{i, j, k} \bar{T}_{i j}^{(k)} & =\sum_{i, j, k} P_{r}\left(\text { user } i \text { transmits using } j^{(k)}\right) \cdot \bar{R}_{i j}^{(k)} \\
& =\sum_{i, j, k} p_{t, i j}^{(k)} \cdot \bar{R}_{i j}^{(k)}
\end{aligned}
$$

subject to two constraints as

$$
\text { s.t. }\left\{\begin{array}{l}
\mathbf{E}\left(P_{i j}^{(k)}\right) \leq P_{a v} \\
\max \left(P_{i j}^{(k)}\right) \leq P_{\max }
\end{array}\right.
$$




\subsubsection{Power Allocation with Channel Inversion}

First, assume power allocation using channel inversion, which means that once the user transmits with channel power gain $h$, the transmit power used is directly given by $\frac{P_{\text {recv }}}{h}$, where $P_{\text {recv }}$ is the equal received power level at every BS.

Then, (4.49) can be represented as

$$
\begin{aligned}
& \bar{R}_{i j}^{(k)}=\frac{\int_{H_{t h}}^{\infty} \iint \cdots \int_{0}^{\alpha H_{t h}} R\left(\gamma\left(h_{1}, h_{2}, \cdots, h_{N}\right)\right) \cdot f_{i j}^{(k)}\left(h_{1}\right) f_{i j_{2}}^{(k)}\left(h_{2}\right) \cdots f_{i j_{N}}^{(k)}\left(h_{N}\right) \mathrm{d} h_{1} \cdots \mathrm{d} h_{N}}{\left[1-F_{i j}^{(k)}\left(H_{t h}\right)\right] \cdot F_{i j_{2}}^{(k)}\left(\alpha H_{t h}\right) F_{i j_{3}}^{(k)}\left(\alpha H_{t h}\right) \cdots F_{i j_{N}}^{(k)}\left(\alpha H_{t h}\right)} \\
& \int_{H_{t h}}^{\infty} \iint \cdots \int_{0}^{\alpha H_{t h}} \frac{W}{L} \cdot \log \left(1+\frac{\tilde{h}_{j j}^{(k)} \cdot P\left(\tilde{h}_{j j}^{(k)}\right)}{N_{0} \cdot \frac{W}{L}+\sum_{l \neq j} \tilde{h}_{l j}^{(k)} \cdot P\left(\tilde{h}_{l l}^{(k)}\right)}\right) \cdot f_{i j}^{(k)}\left(h_{1}\right) f_{i j_{2}}^{(k)}\left(h_{2}\right) \cdots f_{i j_{N}}^{(k)}\left(h_{N}\right) \mathrm{d} h_{1} \cdots \mathrm{d} h_{N} \\
& {\left[1-F_{i j}^{(k)}\left(H_{t h}\right)\right] \cdot F_{i j_{2}}^{(k)}\left(\alpha H_{t h}\right) F_{i j_{3}}^{(k)}\left(\alpha H_{t h}\right) \cdots F_{i j_{N}}^{(k)}\left(\alpha H_{t h}\right)} \\
& =\frac{W}{L} \cdot \frac{\int_{H_{t h}}^{\infty} \iint \cdots \int_{0}^{\alpha H_{t h}} \log \left(1+\frac{P_{\text {recv }}}{N_{0} \cdot \frac{W}{L}+\sum_{l \neq j} \tilde{h}_{l j}^{(k)} \cdot \frac{P_{r e c v}}{\tilde{h}_{h l}^{(k)}}}\right) \cdot f_{i j}^{(k)}\left(h_{1}\right) f_{i j_{2}}^{(k)}\left(h_{2}\right) \cdots f_{i j_{N}}^{(k)}\left(h_{N}\right) \mathrm{d} h_{1} \cdots \mathrm{d} h_{N}}{\left[1-F_{i j}^{(k)}\left(H_{t h}\right)\right] \cdot F_{i j_{2}}^{(k)}\left(\alpha H_{t h}\right) F_{i j_{3}}^{(k)}\left(\alpha H_{t h}\right) \cdots F_{i j_{N}}^{(k)}\left(\alpha H_{t h}\right)} \\
& =\frac{W}{L} \cdot \mathbf{E}\left[\log \left(1+\frac{P_{\text {recv }}}{N_{0} \cdot \frac{W}{L}+\sum_{l \neq j} \tilde{h}_{l j}^{(k)} \cdot \frac{P_{\text {rec }}}{\tilde{h}_{l l}^{(k)}}}\right)\right] \text {. } \\
& \geq \frac{W}{L} \cdot \log \left(1+\frac{P_{r e c v}}{N_{0} \cdot \frac{W}{L}+\sum_{l \neq j} \frac{\alpha H_{t h}}{H_{t h}} \cdot P_{r e c v}}\right) \cdot \frac{\int_{H_{t h}}^{\infty} \iint \cdots \int_{0}^{\alpha H_{t h}} f_{i j}^{(k)}\left(h_{1}\right) f_{i j_{2}}^{(k)}\left(h_{2}\right) \cdots f_{i j_{N}}^{(k)}\left(h_{N}\right) \mathrm{d} h_{1} \cdots \mathrm{d} h_{N}}{\left[1-F_{i j}^{(k)}\left(H_{t h}\right)\right] \cdot F_{i j_{2}}^{(k)}\left(\alpha H_{t h}\right) F_{i j_{3}}^{(k)}\left(\alpha H_{t h}\right) \cdots F_{i j_{N}}^{(k)}\left(\alpha H_{t h}\right)} \\
& =\frac{W}{L} \cdot \log \left[1+\frac{P_{\text {recv }}}{N_{0} \cdot \frac{W}{L}+\alpha \cdot(N-1) P_{\text {recv }}}\right] \\
& \sum_{i, j, k} \bar{T}_{i j}^{(k)}=\sum_{i, j, k} p_{t, i j}^{(k)} \cdot \bar{R}_{i j}^{(k)} \\
& \geq \sum_{i, j, k} p_{t, i j}^{(k)} \cdot \frac{W}{L} \cdot \log \left[1+\frac{P_{\text {recv }}}{N_{0} \cdot \frac{W}{L}+\alpha \cdot(N-1) P_{\text {recv }}}\right]
\end{aligned}
$$

Refer to (4.3), similarly, we can get the average $p_{t, i j}^{(k)}$ as

$$
\bar{p}_{t, i j}^{(k)}=\frac{1}{M} \cdot\left[1-\left(1-p_{a, 1 j}^{(k)}\right)\left(1-p_{a, 2 j}^{(k)}\right) \cdots\left(1-p_{a, M j}^{(k)}\right)\right] .
$$


(4.53) can be further reformulated as

$$
\begin{aligned}
\sum_{i, j, k} \bar{T}_{i j}^{(k)} & \geq \sum_{i, j, k} p_{t, i j}^{(k)} \cdot \frac{W}{L} \cdot \log \left[1+\frac{P_{\text {recv }}}{N_{0} \cdot \frac{W}{L}+\alpha \cdot(N-1) P_{\text {recv }}}\right] \\
& =\sum_{j, k} M \cdot \bar{p}_{t, i j}^{(k)} \cdot \frac{W}{L} \cdot \log \left[1+\frac{P_{\text {recv }}}{N_{0} \cdot \frac{W}{L}+\alpha \cdot(N-1) P_{\text {recv }}}\right] \\
& =\sum_{j, k} M \cdot \frac{1}{M} \cdot\left[1-\prod_{l=1}^{M}\left(1-p_{a, l j}^{(k)}\right)\right] \cdot \frac{W}{L} \cdot \log \left[1+\frac{P_{\text {recv }}}{N_{0} \cdot \frac{W}{L}+\alpha \cdot(N-1) P_{\text {recv }}}\right] \\
& =\sum_{j, k}\left[1-\prod_{l=1}^{M}\left(1-p_{a, l j}^{(k)}\right)\right] \cdot \frac{W}{L} \cdot \log \left[1+\frac{P_{\text {recv }}}{N_{0} \cdot \frac{W}{L}+\alpha \cdot(N-1) P_{\text {recv }}}\right] .
\end{aligned}
$$

Here, $\prod_{l=1}^{M}\left(1-p_{a, l j}^{(k)}\right)$ in the first item of $(4.55)$ is a monotone decreasing function of $M$. We can prove it in this way: assume we have $M$ users distributed within this area at the beginning, then one user jumps into this area. Now we have $M+1$ users, we need to consider the value of $\prod_{l=1}^{M+1}\left(1-p_{a, l j}^{(k)}\right)$. Obviously, this value is less than $\prod_{l=1}^{M}\left(1-p_{a, l j}^{(k)}\right)$ because $\left(1-p_{a,(M+1) j}^{(k)}\right)$ is less than one. This process can be repeated again and again to make the difference between this item and zero as small as possible, that is:

$$
\lim _{M \rightarrow \infty} \prod_{l=1}^{M}\left(1-p_{a, l j}^{(k)}\right)=0 .
$$

Based on (4.56), we can easily get 


$$
\begin{aligned}
\lim _{M \rightarrow \infty} \frac{\sum_{i, j, k} \bar{T}_{i j}^{(k)}}{\sum_{i, k} \bar{T}_{i 1}^{(k)}} & \geq \frac{\lim _{M \rightarrow \infty} \sum_{j, k}\left[1-\prod_{l=1}^{M}\left(1-p_{a, l j}^{(k)}\right)\right] \cdot \frac{W}{L} \cdot \log \left[1+\frac{P_{\text {recv }}}{N_{0} \cdot \frac{W}{L}+\alpha \cdot(N-1) P_{\text {recv }}}\right]}{W \cdot \log \left(1+\frac{P_{\text {recv }}}{N_{0} \cdot \frac{W}{L}}\right)} \\
& =N .
\end{aligned}
$$

Combining (4.57) and (4.28), we can easily get the same result as we have got in the scenario of homogeneous network model. That is: $N$ times of the throughput of a single BS.

\subsubsection{Power Allocation with Equality}

Similarly, we can get the result of power allocation with equality based on (4.55) as follows:

$$
\sum_{i, j, k} \bar{T}_{i j}^{(k)} \geq \sum_{j, k}\left[1-\prod_{l=1}^{M}\left(1-p_{a, l j}^{(k)}\right)\right] \cdot \frac{W}{L} \cdot \log \left[1+\frac{H_{t h} \cdot P}{N_{0} \cdot \frac{W}{L}+(N-1) \alpha H_{t h} P}\right]
$$

\subsection{Summary}

In this chapter, we gave a detailed mathematical analysis of our system model. To achieve sum throughput maximization, we have proved that in both homogeneous and heterogeneous networks we can achieve $N$ (the number of BSs) times of that of centralized scheduling if the number of users goes to infinity. This builds the foundation for our distributed multi-channel multi-cell scheduling algorithm. By exploiting multiuser diversity, we can achieve reasonable performance while using lessening the load of BSs. 


\section{Chapter 5}

\section{Simulation and Numerical Results}

\subsection{Simulation Platform Setup}

We consider a multi-cell network with four BSs within a $2000 \times 2000 \mathrm{~m}^{2}$ area. Different numbers of wireless terminals are distributed randomly within this area.

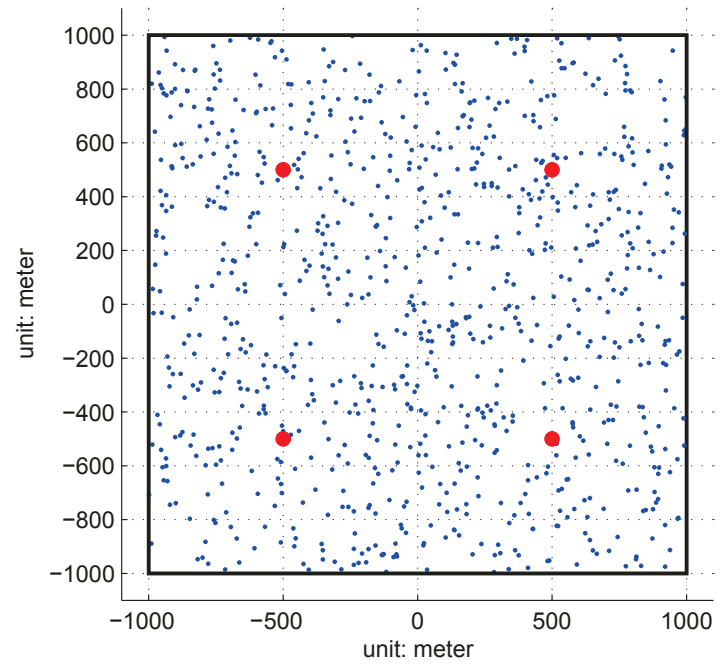

Figure 5.1: A multi-cell network with four BSs. ${ }^{1}$

For the path loss, we use 3GPP LTE Urban macrocell model (approximately $1 \mathrm{~km}$ distance from BS to BS) [34]. For the small-scale fading, we use Rayleigh fading, that

\footnotetext{
${ }^{1}$ Note: big red points represent $4 \mathrm{BS} s$, small blue points represent wireless terminals.
} 
is, exponential distribution for channel power gain. The related simulation parameters are summarized in Table 5.1.

Table 5.1: Summary of simulation parameters for Scenario I.

\begin{tabular}{c|l}
\hline \hline The distance between mobile terminals and BSs $(\mathrm{m})$ & $d$ \\
\hline Path Loss (PL) & $34.5+35 \log (d)$ (Macro Urban LTE model, assuming $d \geq 35 \mathrm{~m})$ \\
\hline Channel Power Gain & Rayleigh Fading: exprnd(1) \\
\hline BS Antenna Gain & $18 \mathrm{dBi}$ \\
\hline Transmit Power & $0.025 \mathrm{~W}=14 \mathrm{dBm}$ \\
\hline No. of BSs & 4 \\
\hline No. of RBs & 60 \\
\hline Reuse factor & $1($ all RBs are used by each BS) \\
\hline Bandwidth per RB & $180 \mathrm{KHz}$ \\
\hline Power Spectral Density of Noise $\left(N_{0}\right)$ & $4 \times 10^{-21} \mathrm{~W} / \mathrm{Hz}$ \\
\hline No. of wireless terminals & $60,120,240,480$ \\
\hline
\end{tabular}

\subsection{Simulation Results for Scenario I: Sum Throughput Maximization}

Our simulation mainly focuses on the two performance indexes: one is the sum throughput, the other is the number of candidate users.

\subsubsection{Sum Throughput Maximization}

Our simulation compares the performance of the following schemes:

1) Distributed Scheduling (DS)

Using "Algorithm I" discussed earlier. 
2) Distributed Scheduling (DS2)

A small change to "Algorithm I" in that we use one threshold for the summation of interference channels, while in "Algorithm I", we use one threshold for a single interference channel.

3) W.-Y. Shin scheme (SH)

Algorithm discussed in [28], similar to "DS2", except that no criterion value is used at the BS for selecting users to transmit. Users are selected randomly at the BS among all the candidate users.

4) Centralized Scheduling (CS)

Users with the best channels are selected by BS among all users and no interference needs to be considered.

In our simulation, we select from all the users the "best" one for every BS. To incorporate the effect of multiuser diversity, we assume that for every RB time period, one user can only select the BS with the best channel then try to contend for the RB of the BS.

We simulate the performance of different schemes using channel power gain threshold $H_{t h}$ from $-160 \mathrm{~dB}$ to $-70 \mathrm{~dB}$, attenuation factor $\alpha$ from $0 \mathrm{~dB}$ to $-50 \mathrm{~dB}$ for different number of users (from 60 to 480). The simulation results are shown in the following figures. 


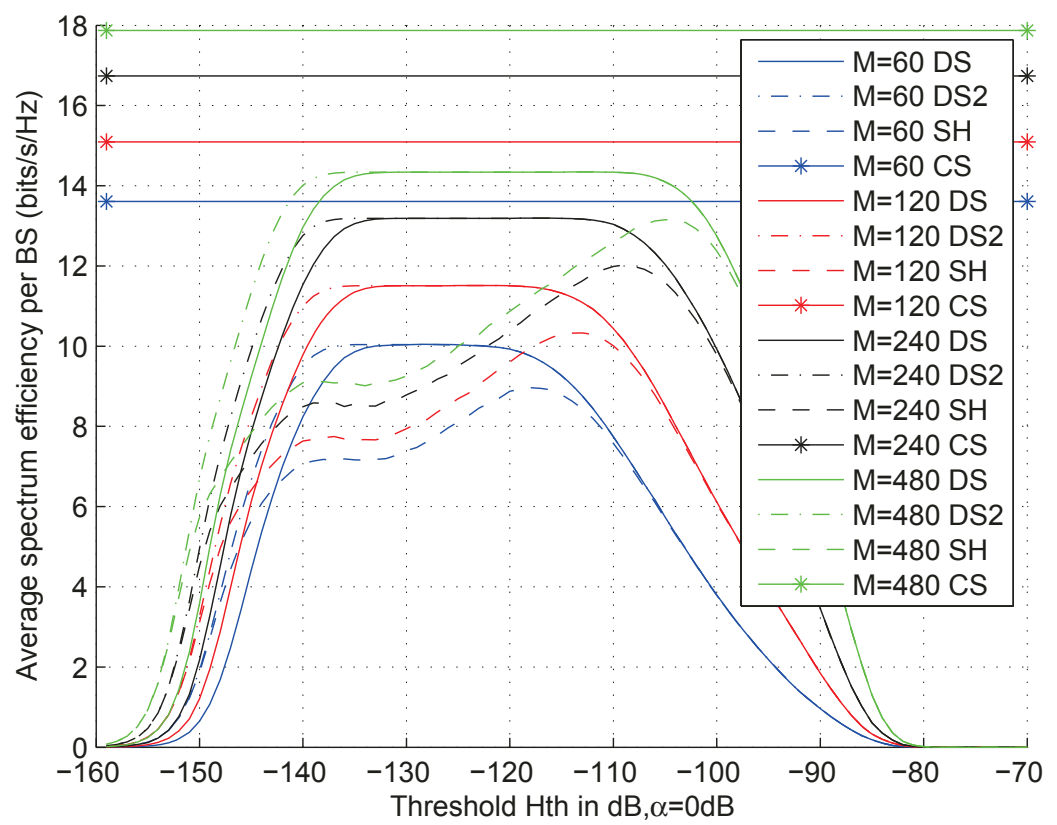

Figure 5.2: Average spectral efficiency per BS over all RBs $(\alpha=0 \mathrm{~dB})$.

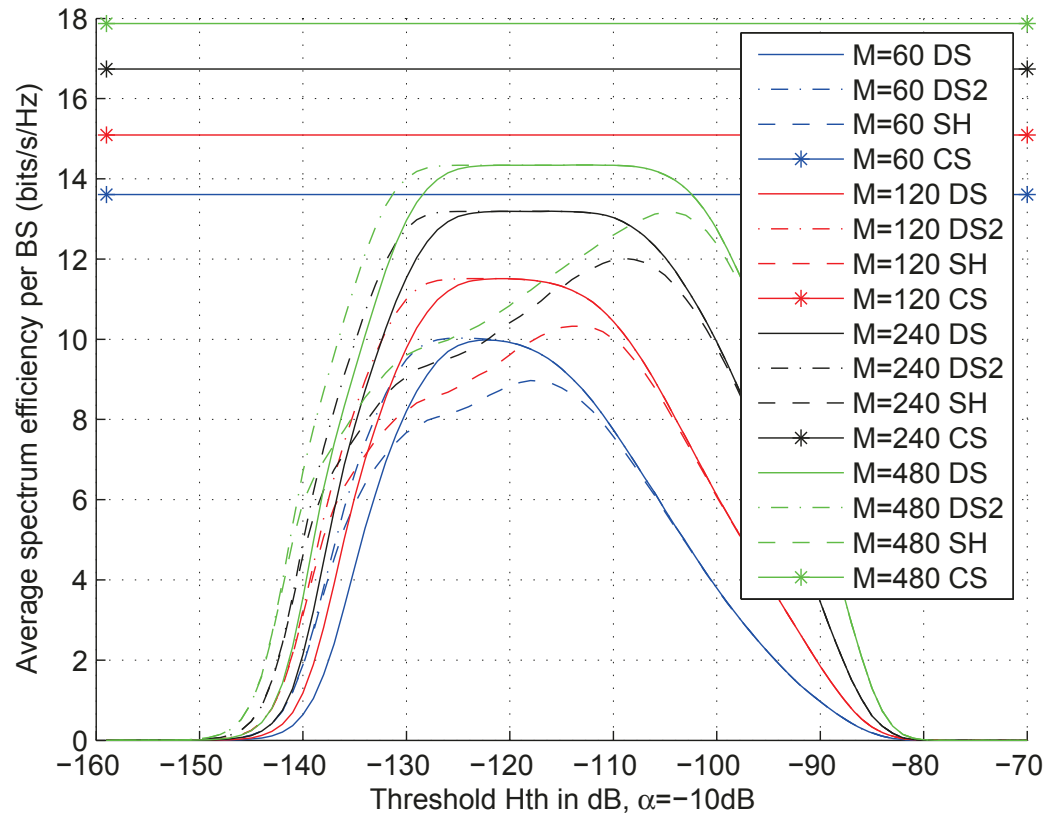

Figure 5.3: Average spectral efficiency per BS over all RBs $(\alpha=-10 \mathrm{~dB})$. 


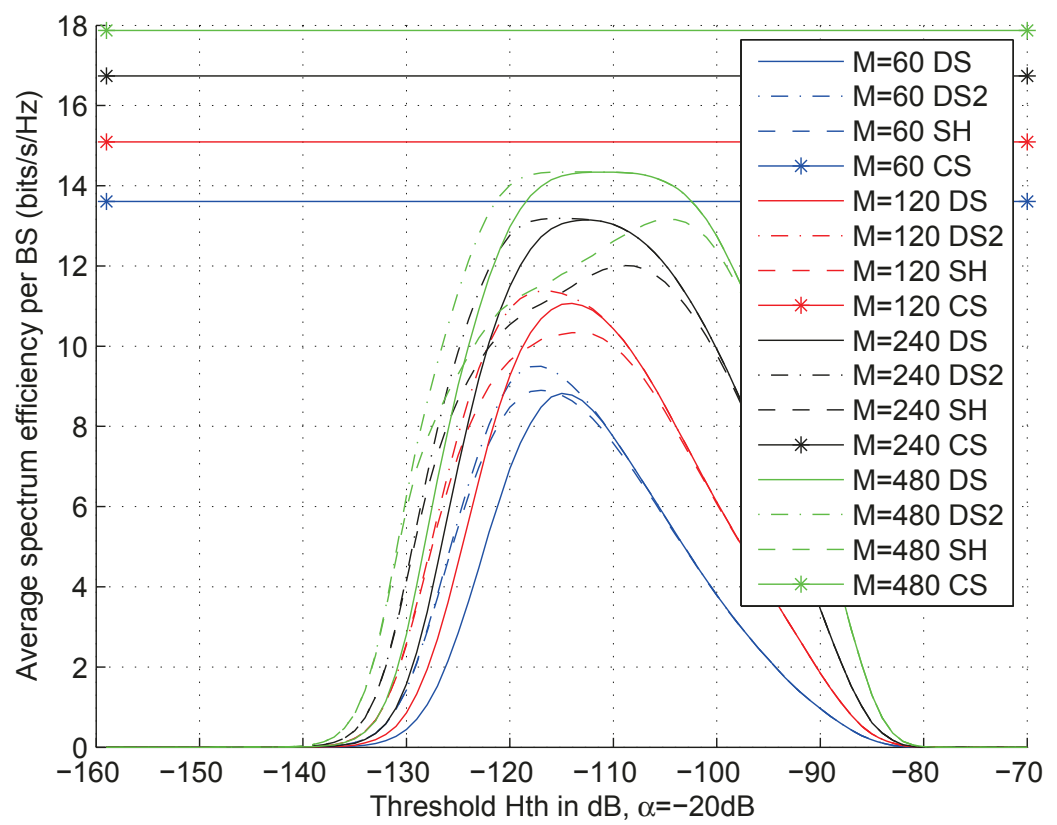

Figure 5.4: Average spectral efficiency per BS over all RBs $(\alpha=-20 \mathrm{~dB})$.

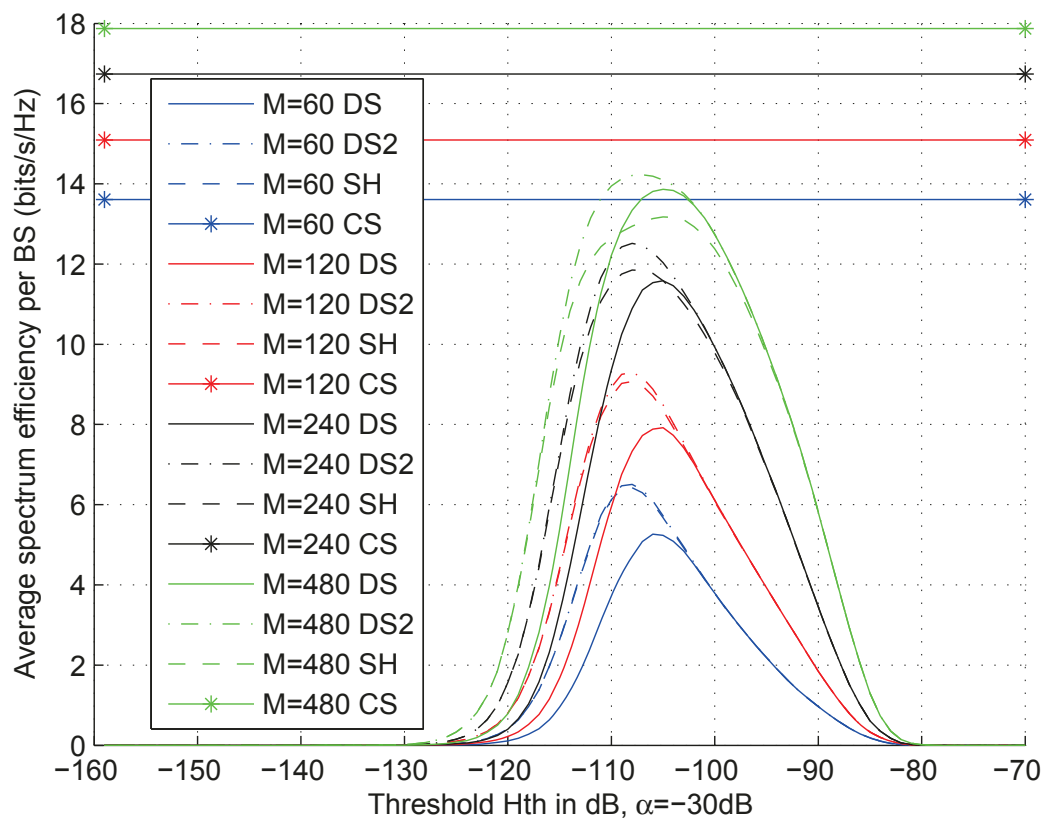

Figure 5.5: Average spectral efficiency per BS over all RBs $(\alpha=-30 \mathrm{~dB})$. 


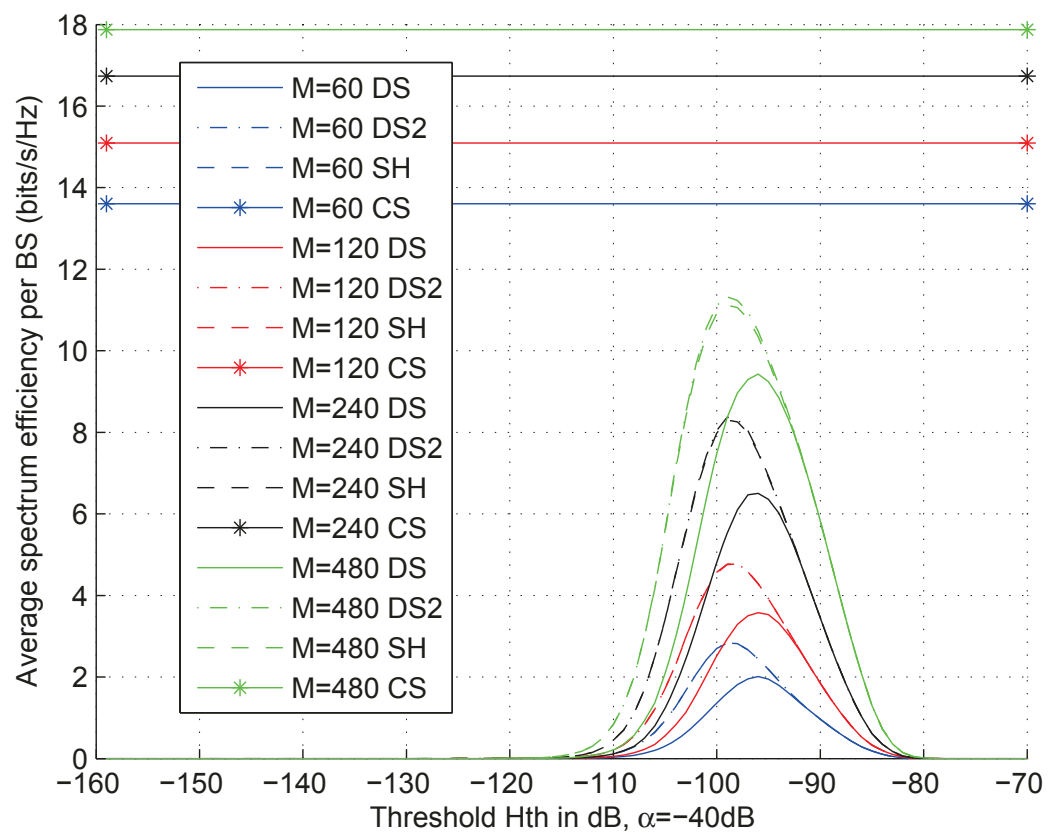

Figure 5.6: Average spectral efficiency per BS over all RBs $(\alpha=-40 \mathrm{~dB})$.

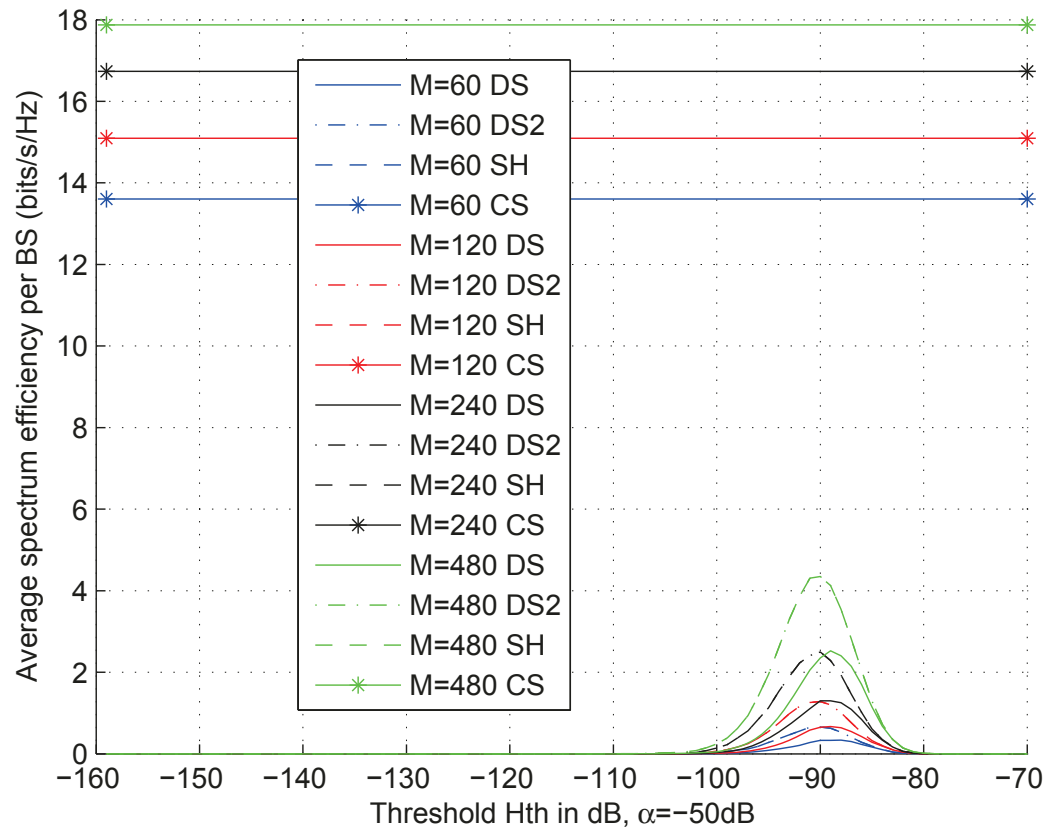

Figure 5.7: Average spectral efficiency per BS over all RBs $(\alpha=-50 \mathrm{~dB})$. 
Figures 5.2-5.7 illustrate the comparison of spectral efficiency per BS between DS, DS2, SH, CS with different number of users and attenuation factors. Here, it should be noted that we calculate the spectral efficiency of centralized scheduling by selecting the best channels of all the channel coefficients from all the users. In this way, we will calculate total throughput of four BSs and get the average throughput per BS. Based on average throughput per BS, we can get spectral efficiency of centralized scheduling. All the values are the average results of 100 simulations.

1) It can be easily seen that multi-user diversity plays an important role in both distributed scheduling and centralized scheduling. Spectral efficiency increases with the number of users for both centralized scheduling and distributed scheduling. For example, in both cases, the curves of $M=480$ are above those of $M=240$.

2) Compared to centralized scheduling, distributed scheduling achieves a very good performance. The ratio of spectral efficiency of distributed scheduling to centralized scheduling is near 0.78 when the number of users is 480 . Note that in our simulation, calculation of spectral efficiency using CS scheme actually exploits multi-user diversity to some extent because we select "best" users for different BSs from all the users within the whole area. So in reality this value will increase. This performance can also be attributed to the fading effect. Because of fading and the assumption of uncorrelation of different links, we still can get reasonable performance. Our algorithm exploits this effect and select those users with better CSI while causing little interferences to other users. It should also be noted that we use Rayleigh flat fading in our simulation which is very ideal for exploiting fading effect since zero interference gets the highest probability density.

3) For the performance of different distributed scheduling schemes, DS2 gets a 
better spectral efficiency than DS or at least equal to DS and DS is better than $\mathrm{SH}$ in most cases except at low threshold $H_{t h}$ region. This is because the constraint for interference links in DS2 is looser than DS, which means that candidate users in DS are always part of those in DS2. This leads to a larger selection pool of candidate users in DS2. For this reason, DS2 is more likely to select better users than DS. But with the value of the thresholds increases, the difference between DS2 and DS goes to very smaller (this is shown by Figures 5.2-5.7) and overlaps in the range of high threshold values. This is mainly because that the difference on the number of candidate users is much smaller with the increase of threshold values.For SH, it is based on random selection without considering criterion values. That's why we get worse performance.

4) Although the number of users may be different, it should be noted that the thresholds for achieving peak value are almost the same and this threshold changed with the changing of $\alpha$. We can see that the curves shift to right with the decreasing of $\alpha$. This value is obvious closely related with the characteristics of channels since this is mainly decided by the ratio of communication channel to the summation of interference channels. 


\subsubsection{The Number of Candidate Users}

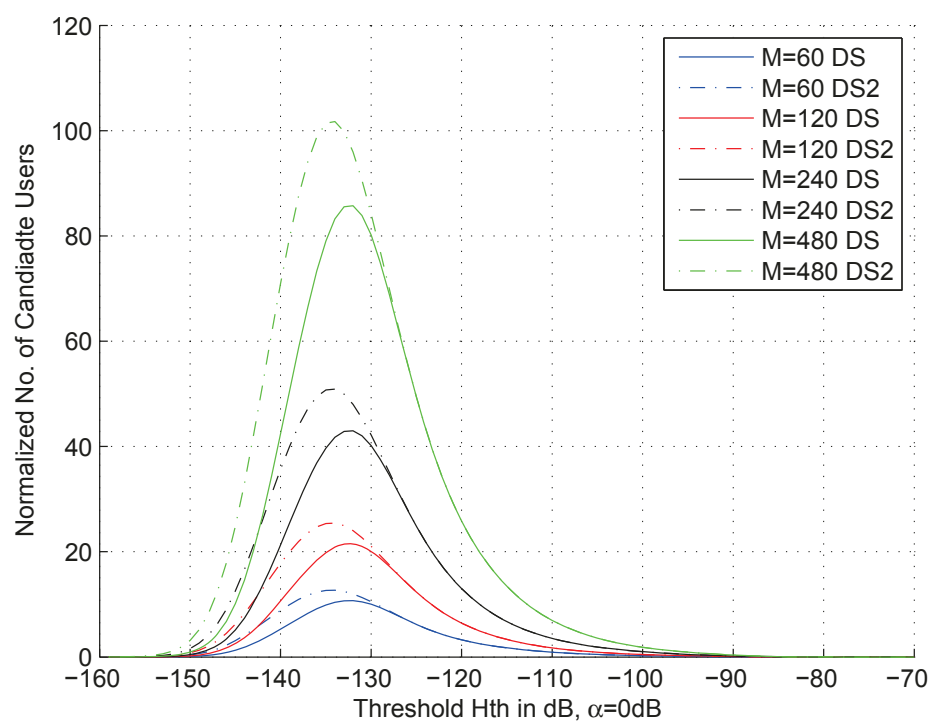

Figure 5.8: Normalized no. of candidate users per RB $(\alpha=0 \mathrm{~dB})$.

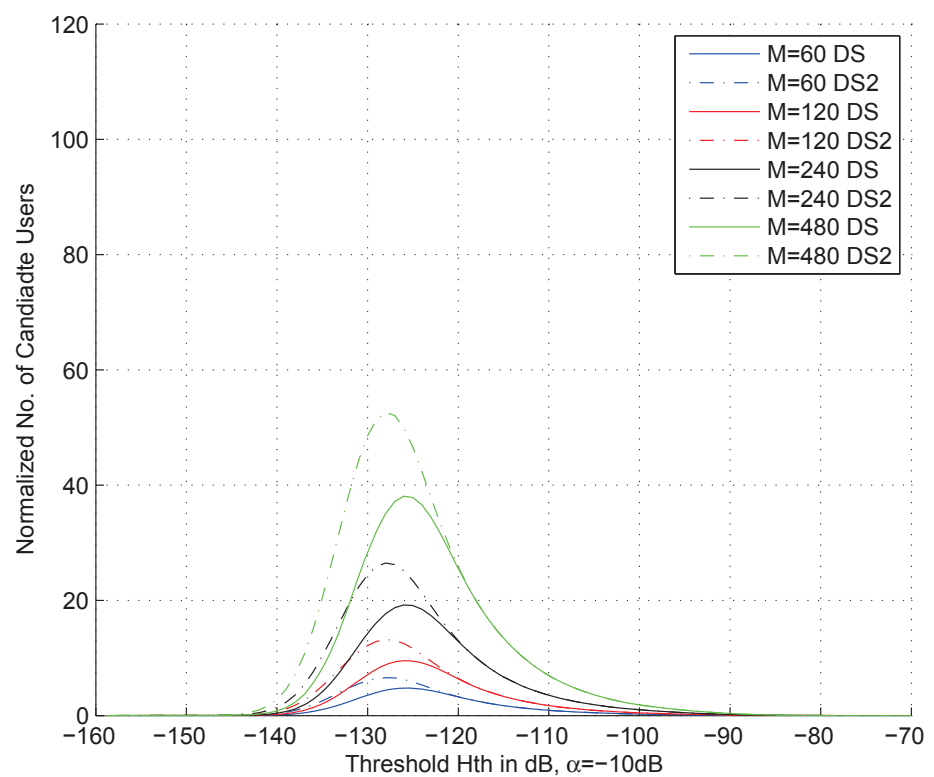

Figure 5.9: Normalized no. of candidate users per RB ( $\alpha=-10 \mathrm{~dB})$. 


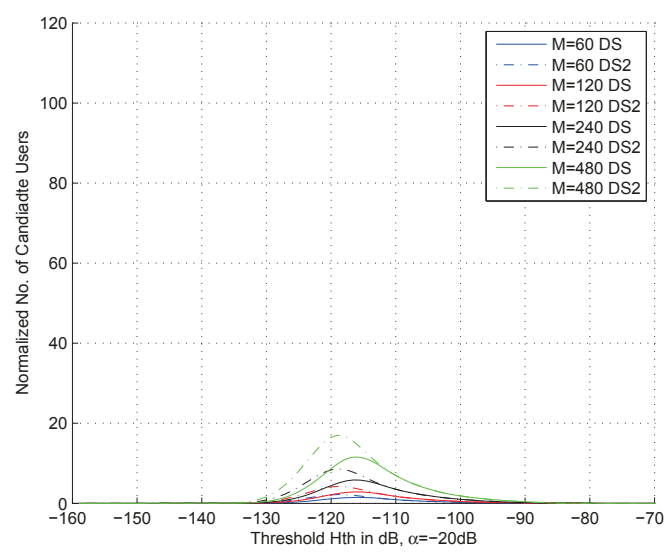

(a) $\alpha=-20 \mathrm{~dB}$.

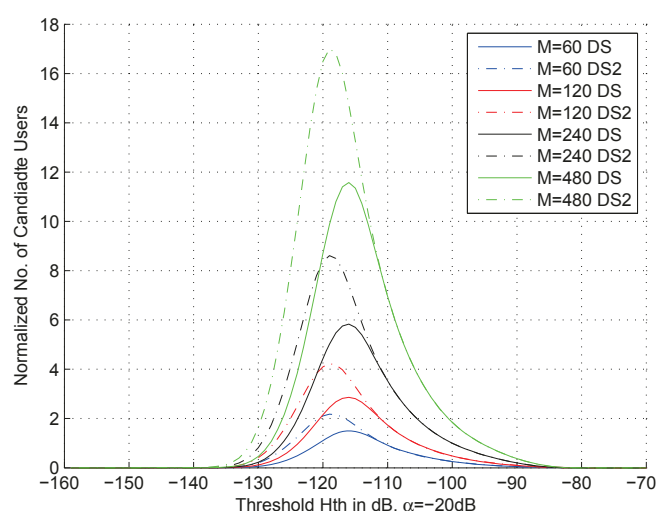

(b) $\alpha=-20 \mathrm{~dB}$ (magnified).

Figure 5.10: Normalized no. of candidate users per RB $(\alpha=-20 \mathrm{~dB})$.

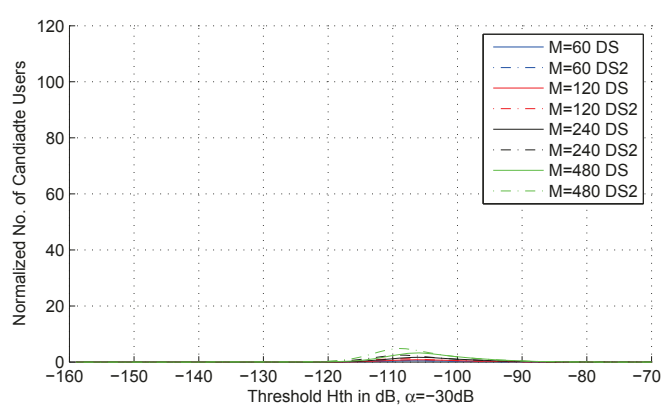

(a) $\alpha=-30 \mathrm{~dB}$.

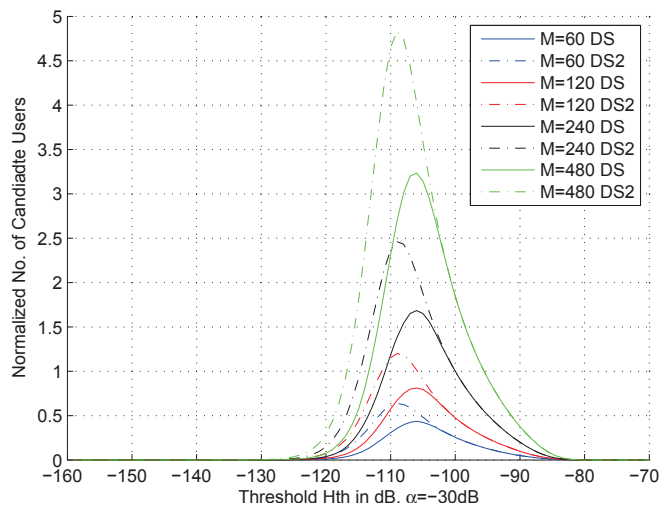

(b) $\alpha=-30 \mathrm{~dB}$ (magnified).

Figure 5.11: Normalized no. of candidate users per RB $(\alpha=-30 \mathrm{~dB})$. 


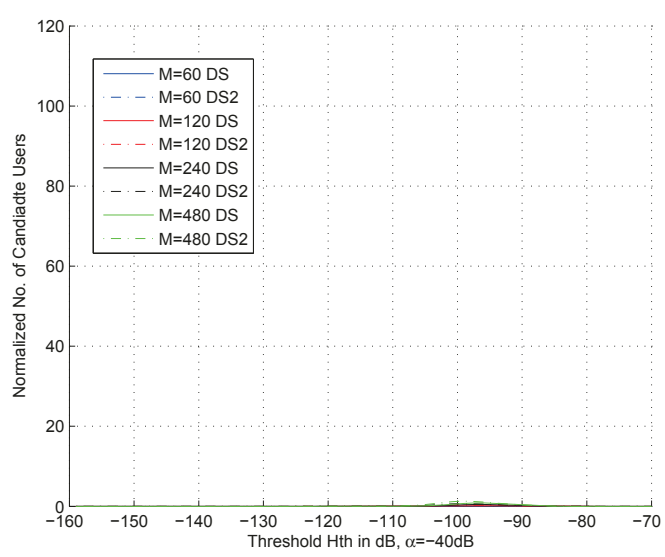

(a) $\alpha=-40 \mathrm{~dB}$

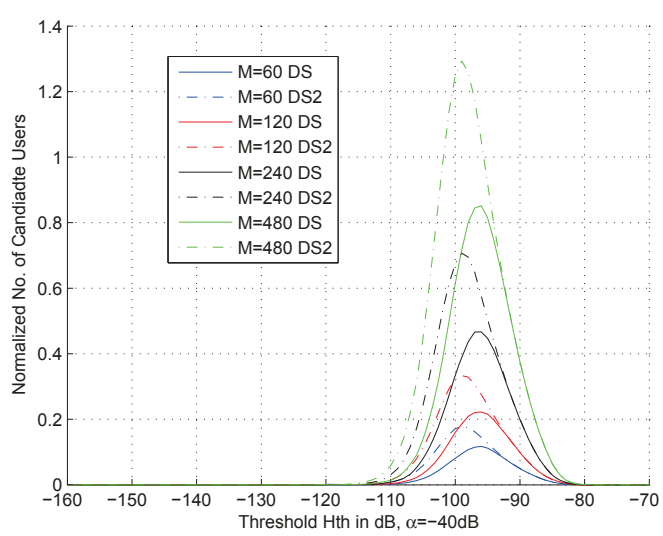

(b) $\alpha=-40 \mathrm{~dB}$ (magnified).

Figure 5.12: Normalized no. of candidate users per RB $(\alpha=-40 \mathrm{~dB})$.

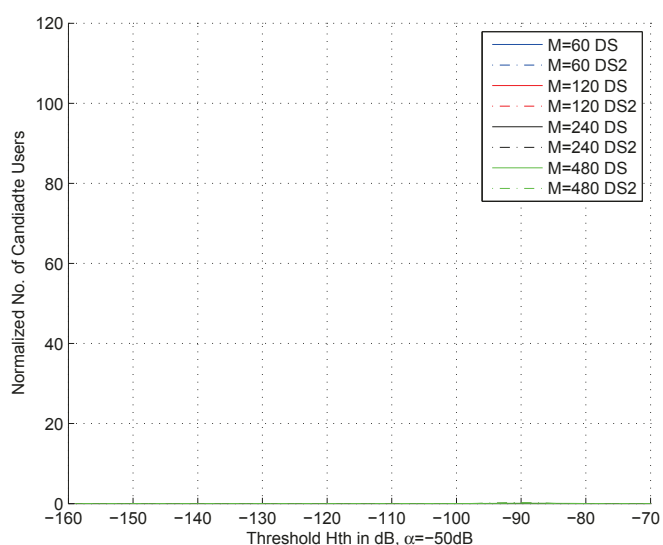

(a) $\alpha=-50 \mathrm{~dB}$.

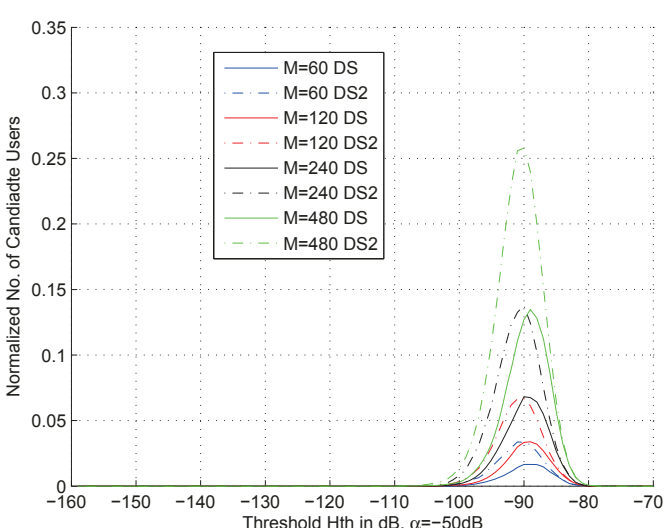

(b) $\alpha=-50 \mathrm{~dB}$ (magnified).

Figure 5.13: Normalized no. of candidate users per RB $(\alpha=-50 \mathrm{~dB})$.

Here, we use candidate users to represent those users who will send channel requests to compete for RBs. "Normalized number of candidate users" represents that on average the number of channel requests per RB. Figures 5.8-5.13 illustrate the relationship of normalized number of candidate users to threshold $H_{t h}$ and attenuation factor $\alpha$.

1) Attenuation factor is necessary to control the number of candidate users and 
this is clearly demonstrated by Figure 5.8, where attenuation factor $\alpha$ is $0 \mathrm{~dB}$. Normalized number of candidate users is very large at low threshold area, this means that a large amount of control channels need to be configured to accommodate this. This is obvious a waste of radio resources since only a very small number of users will get the RBs.

2) It should also be noted that for same scheduling schemes the thresholds for achieving peak values of normalized number of candidate users are almost same as for achieving peak values of throughput, although thresholds for achieving peak values using different schemes may be different. For example, under the same value of $H_{t h}$ and $\alpha$, the thresholds for getting peak values of spectral efficiency using DS2 is always a little less than those using DS. The same rule can be found for the thresholds of getting peak values of normalized number of candidate users. This is another example that shows the effects of multiuser diversity.

3) By adjusting $H_{t h}$, attenuation factor $\alpha$, we can control the number of candidate users, and this is related to the configuration of control channel resources. The fundamental principle in selecting the threshold $H_{t h}$ and attenuation factor $\alpha$ is to ensure that the waste of RBs should be avoided.

\subsubsection{Spectral Efficiency with Different Power Allocation}

All the above simulations use fixed power allocation, we also conduct simulation under different power allocations (from $-10 \mathrm{dBm}$ to $22 \mathrm{dBm}$ ) to calculate the spectral efficiency, the results can be shown in Figure 5.14. From these results, we can get some insights for criterion value equation used in Algorithm I. 


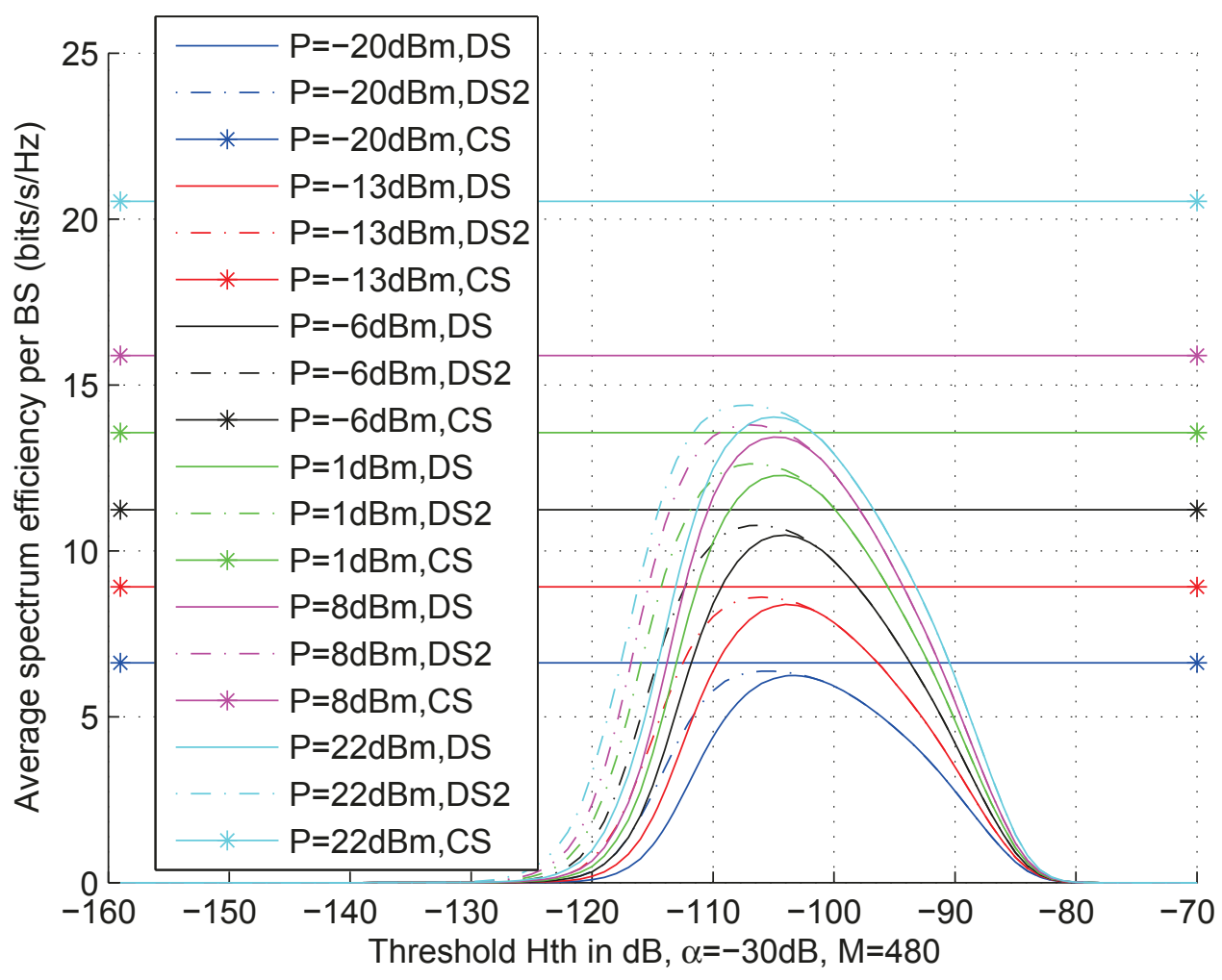

Figure 5.14: Average spectral efficiency per BS with different power allocations.

It can easily be seen that performance (spectral efficiency) increases dramatically with the increase of power when the power is low, but saturates at high power values. That is, at low SINR, it's a power-limited system while at high SINR, it's an interference-limited system. This indirectly justifies our selection of criterion value for Algorithm I. We are using Shannon equation $\log \left(1+\frac{P h_{i j}^{(k)}}{P_{n o i s e}+P \sum_{l \neq i} h_{l j}^{(k)}}\right)$ to estimate the rate. Here, the noise power $P_{\text {noise }}$ is a constant, so when the transmit power is small, this noise power may have more weight in deciding the value of rate but it can be ignored when the transmit power is getting larger. In this case, the power of interference will be much larger than the noise power so the noise power can be ignored. If we assume equal transmit power for all the users, then $\frac{P h_{i j}^{(k)}}{P_{n o i s e}+P \sum_{l \neq i} h_{l j}^{(k)}}$ can be approximated as $\frac{h_{i j}^{(k)}}{\sum_{l \neq i} h_{l j}^{(k)}}$. This is very similar to our proposed criterion value $\frac{h_{i j}^{(k)}}{\sum_{l \neq i} h_{l j}^{(k)}}$ 
except that the denominator is composed of interference links from users of different BSs. Our proposed criterion value tries to select those users who will cause interferences as little as possible. If we select every users by this criterion values, this will

serve to the benefits of $\log \left(1+\frac{P h_{i j}^{(k)}}{P_{n o i s e}+P \sum_{l \neq i} h_{l j}^{(k)}}\right)$ finally. Another issue worthy of note is that the channel power gain of the communication link should be above a certain threshold, otherwise the received power would not be much higher than noise power. This justifies the adoption of threshold $H_{t h}$ in our algorithm.

\subsubsection{Fairness Issue in Scenario I}

In scenario I, our main goal is to get the maximum sum throughput and we do not specially consider the issue of fairness. Usually, there is a tradeoff between efficiency and fairness. To get good fairness means that you will lose some efficiency to some extent. We also examine the fairness in Scenario I. To get a better understanding of fairness in Scenario I, we select a part of our simulation results to calculate Jain's fairness index. We select the threshold range as $-120 \mathrm{~dB}$ to $-95 \mathrm{~dB}$, the number of users is 480 , attenuation factor $\alpha$ is $-30 \mathrm{~dB}$. The simulation is run 300 times. The reason for selecting this configuration is that we can see from Figure 5.5 that all the normalized number of users for scheduling scheme DS, DS2, and SH is approximately more than one in this range. This will make sense for evaluating fairness issue otherwise if no user is selected for transmission, this kind of "fairness" is obviously not what we need to consider. The simulation results are shown in Figure 5.15. 


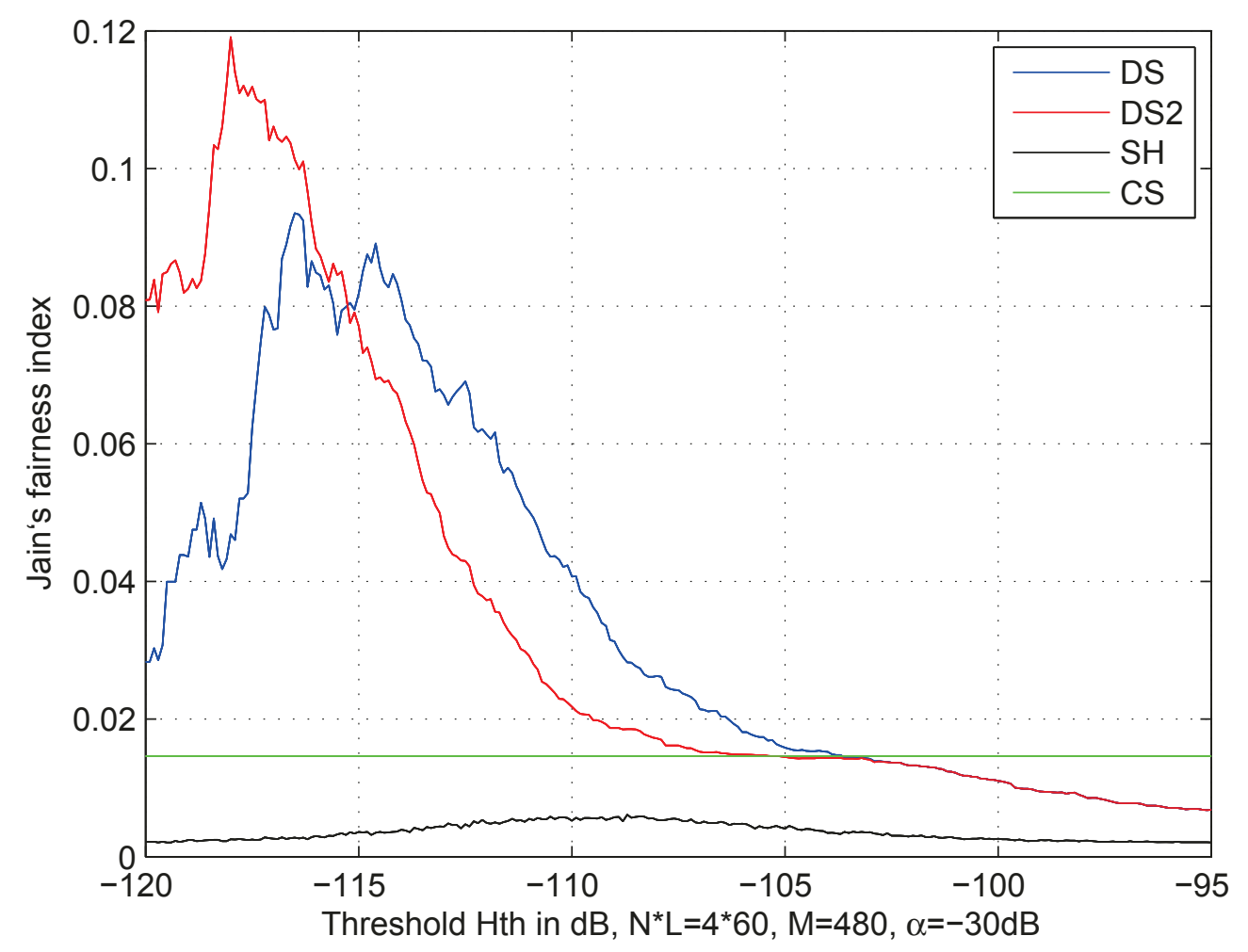

Figure 5.15: Jain's fairness index for schemes DS, DS2, SH, CS.

Here, for centralized scheduling (CS), the calculation is just based on data from 300 times of simulation and this has nothing to do with the value of threshold. To make comparison, we put the result in one figure. It can be seen that all of schemes perform badly when it comes to fairness. The schemes CS and SH perform worse than DS and DS2. It should also be noted that the curves of DS and DS2 overlapped after the threshold value of approximately $-104 \mathrm{~dB}$. This can be demonstrated by Figure 5.5 and Figure 5.11. After this value, the curves of spectral efficiency and normalized number of candidate users are overlapped. We can also get the CDF of the average data rate under different scheduling schemes as shown in Figures 5.165.19. These figures show that only quite a few users can get the chances to transmit. 


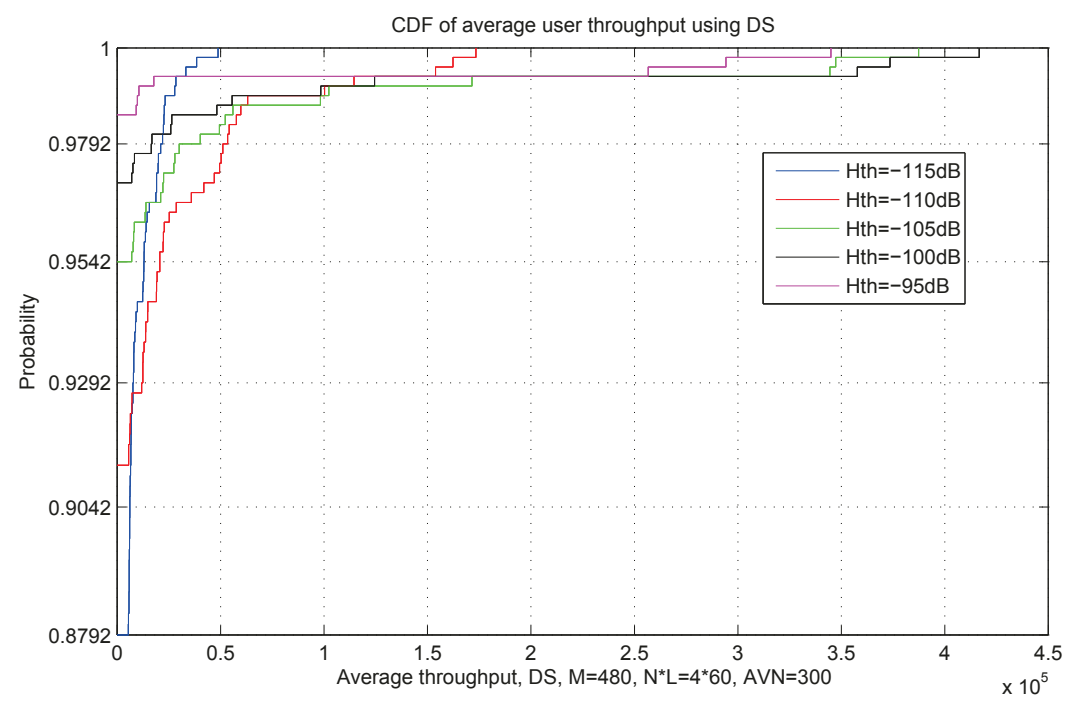

Figure 5.16: CDF of average user data rate using DS scheme.

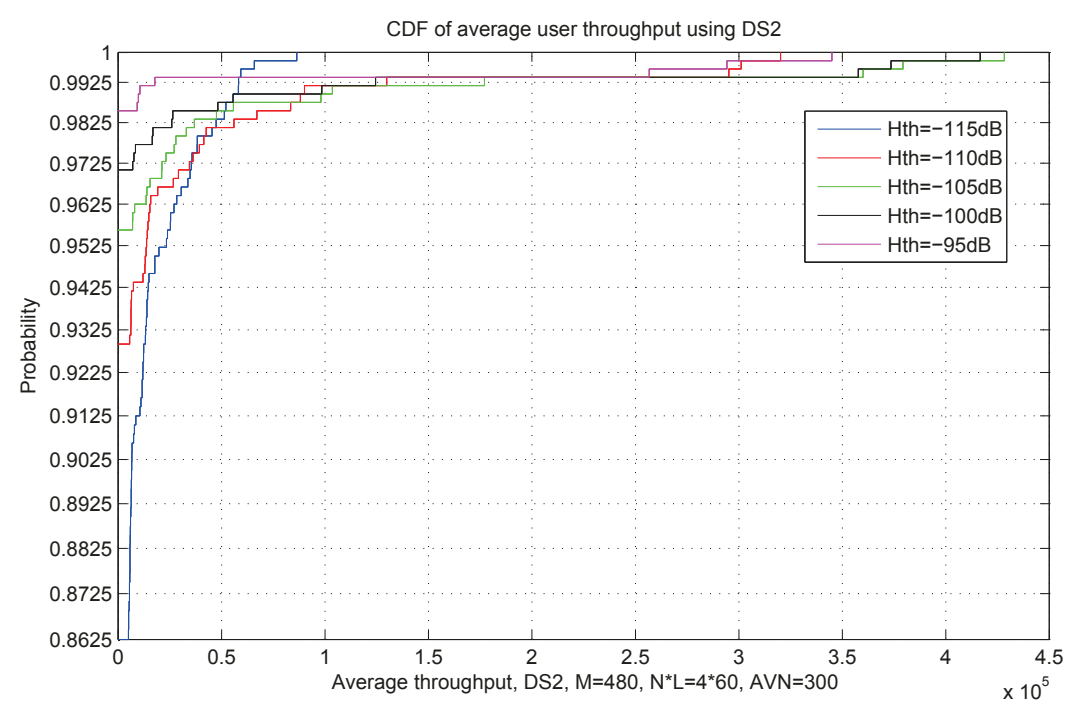

Figure 5.17: CDF of average user data rate using DS2 scheme. 


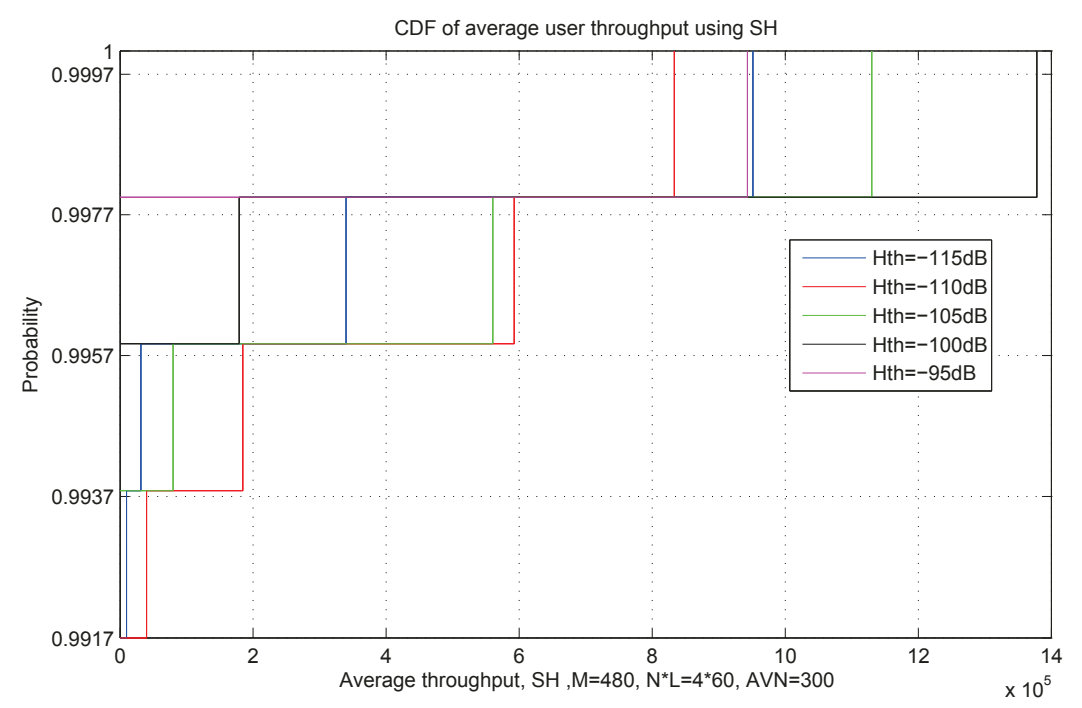

Figure 5.18: CDF of average user data rate using SH scheme.

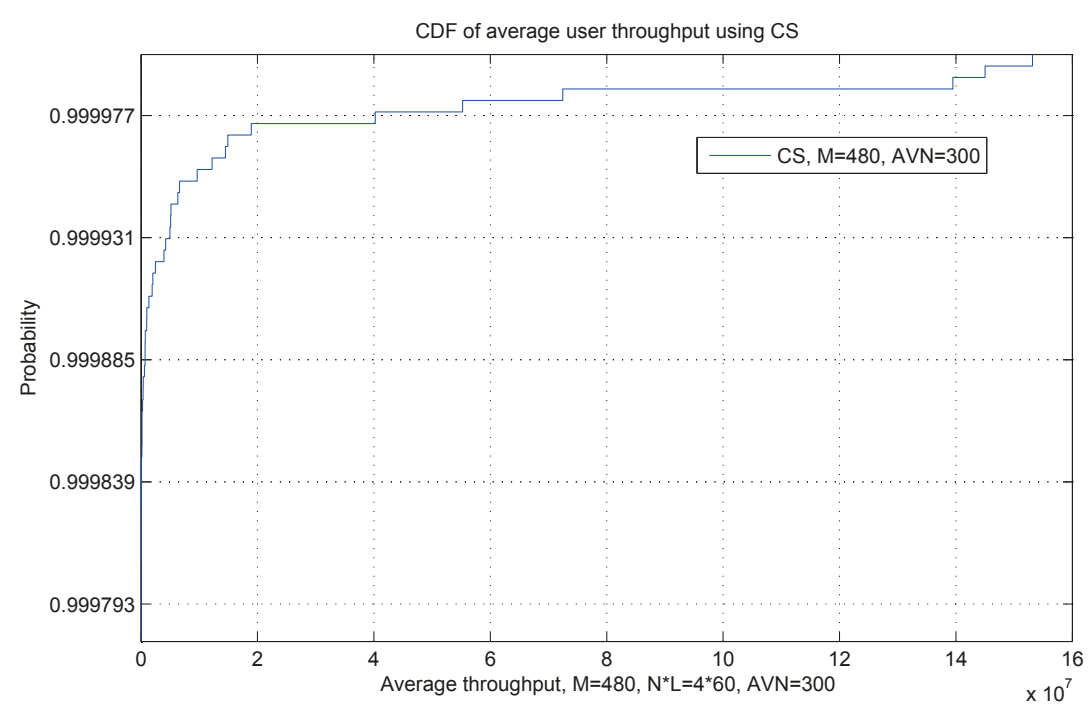

Figure 5.19: CDF of average user data rate using CS scheme.

\subsubsection{Mass Traffic Handling in Scenario I}

If unexpected mass traffic happens, the performance of Algorithm I will be severely influenced. First, the threshold $H_{t h}$ may not be large enough, then the number of channel reservation requests will dramatically increase and the control resources may 
be not enough to handle these large number of requests which lead to BSs' difficulty of decoding these requests and making arbitration. It is better to have BSs adaptive to the changing conditions of networks by broadcasting the adjusted values of $H_{t h}$ and $\alpha$. In this way, BSs can control the number of channel reservation requests and needed control channels without causing great impacts to the networks.

\subsection{Simulation Results for Scenario II: Propor- tional Fairness Approximation}

In this part, we use a scenario of 4 BSs (each has $30 \mathrm{RBs}$ ) with 120 users (represented as "M") for 2000 time periods (represented as "Q") to simulate the calculation of Jain's fairness index at different window sizes (WIN $=30,40,50, \ldots, 100)$. The configuration of simulation parameters can be summarized as Table 5.2. 
Table 5.2: Summary of simulation parameters for Scenario II.

\begin{tabular}{|c|c|}
\hline The distance between mobile terminals and BSs $(\mathrm{m})$ & $d$ \\
\hline Path Loss (PL) & $34.5+35 \log (d)$ (Macro Urban LTE model, assuming $d \geq 35 \mathrm{~m}$ ) \\
\hline Channel Power gain & Rayleigh Fading: exprnd(1) \\
\hline BS Antenna Gain & $18 \mathrm{dBi}$ \\
\hline Transmit Power & $0.025 \mathrm{~W}=14 \mathrm{dBm}$ \\
\hline No. of BSs $(\mathrm{N})$ & 4 \\
\hline No. of RBs (L) & 30 \\
\hline Reuse Factor & 1 \\
\hline Bandwidth per RB & $180 \mathrm{KHz}$ \\
\hline Power Spectral Density of Noise $\left(N_{0}\right)$ & $4 \times 10^{-21} \mathrm{~W} / \mathrm{Hz}$ \\
\hline No. of Time Periods for average rate calculation (WIN) & $30,40,50, \ldots, 100$ \\
\hline No. of Time Periods for simulation (Q) & 2000 \\
\hline No. of wireless terminals (M) & 120 \\
\hline
\end{tabular}

The results can be see in Figure 5.20. It can be seen that we can get quite good performance and this means that we can use "Algorithm II" to approximate proportional fairness in practice. It should be noted that given the same number of time periods greater window sizes lead to smaller values of Jain's fairness index. This is reasonable because smaller window sizes means that those who do not have a chance to transmit in the past time periods (we call this kind of users as "zero channel users") have more chances to transmit than the users in a network with greater window sizes. This is obviously more "fair" considering that every user does not need to wait too long to transmit. But smaller window size is not beneficial to average user rate, since in a network with smaller window size zero channel users have more chance to transmit and their channel conditions are not guaranteed. Thus the overall throughput will be lower in a network with smaller window size. This 
basically shows the tradeoff between fairness and throughput.

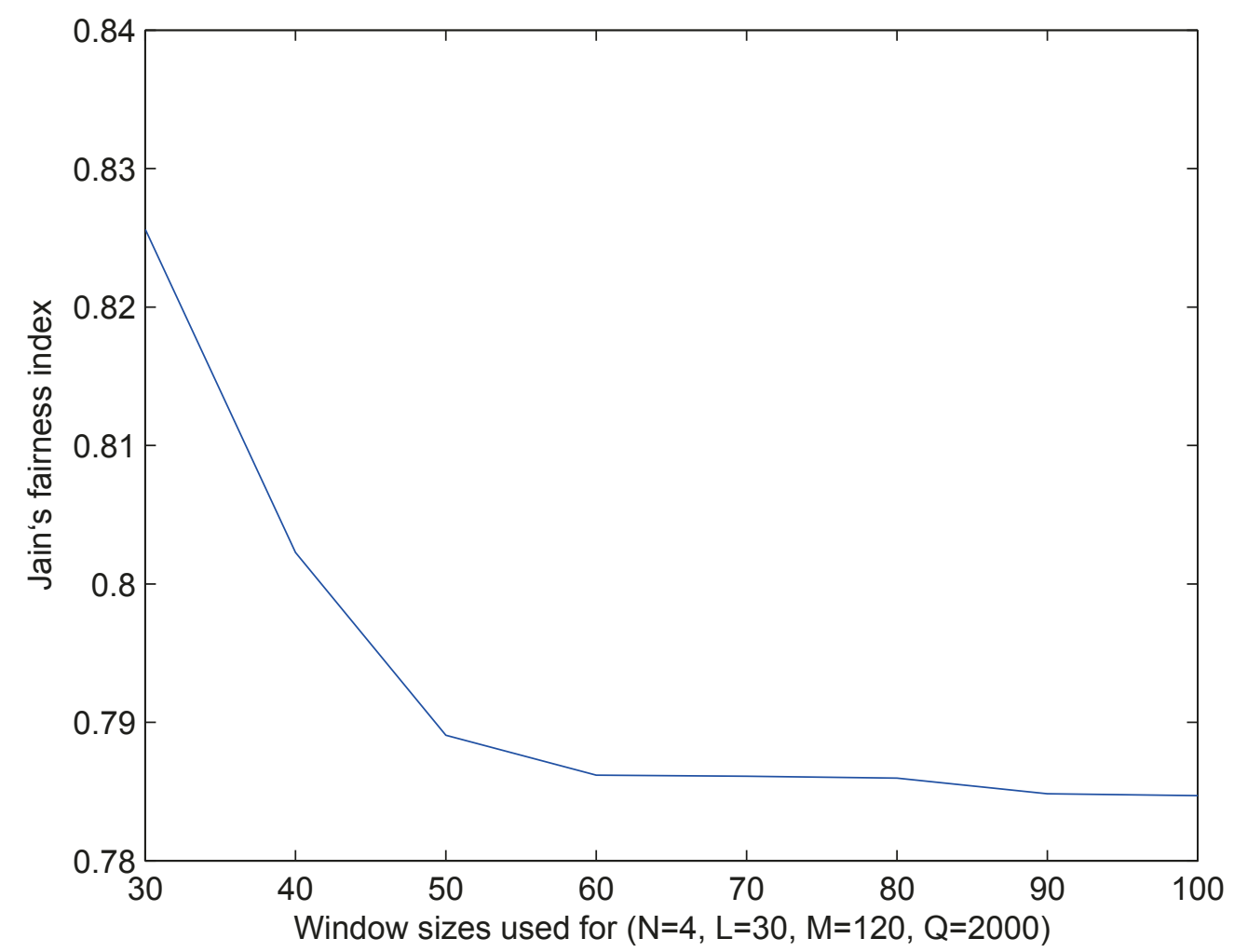

Figure 5.20: Simulation with Jain's fairness index.

\subsubsection{Distribution of the Selected Criterion Values with Dif- ferent Window Sizes}

We also simulate the average selected criterion values (represented as "AV $\left.\left(\mathrm{CV}^{*}\right) "\right)$ at different window sizes $(\mathrm{WIN}=30,40,50, \ldots, 100)$. The configuration of simulation parameters is the same as Table 5.2. The result is shown in Figure 5.21. 


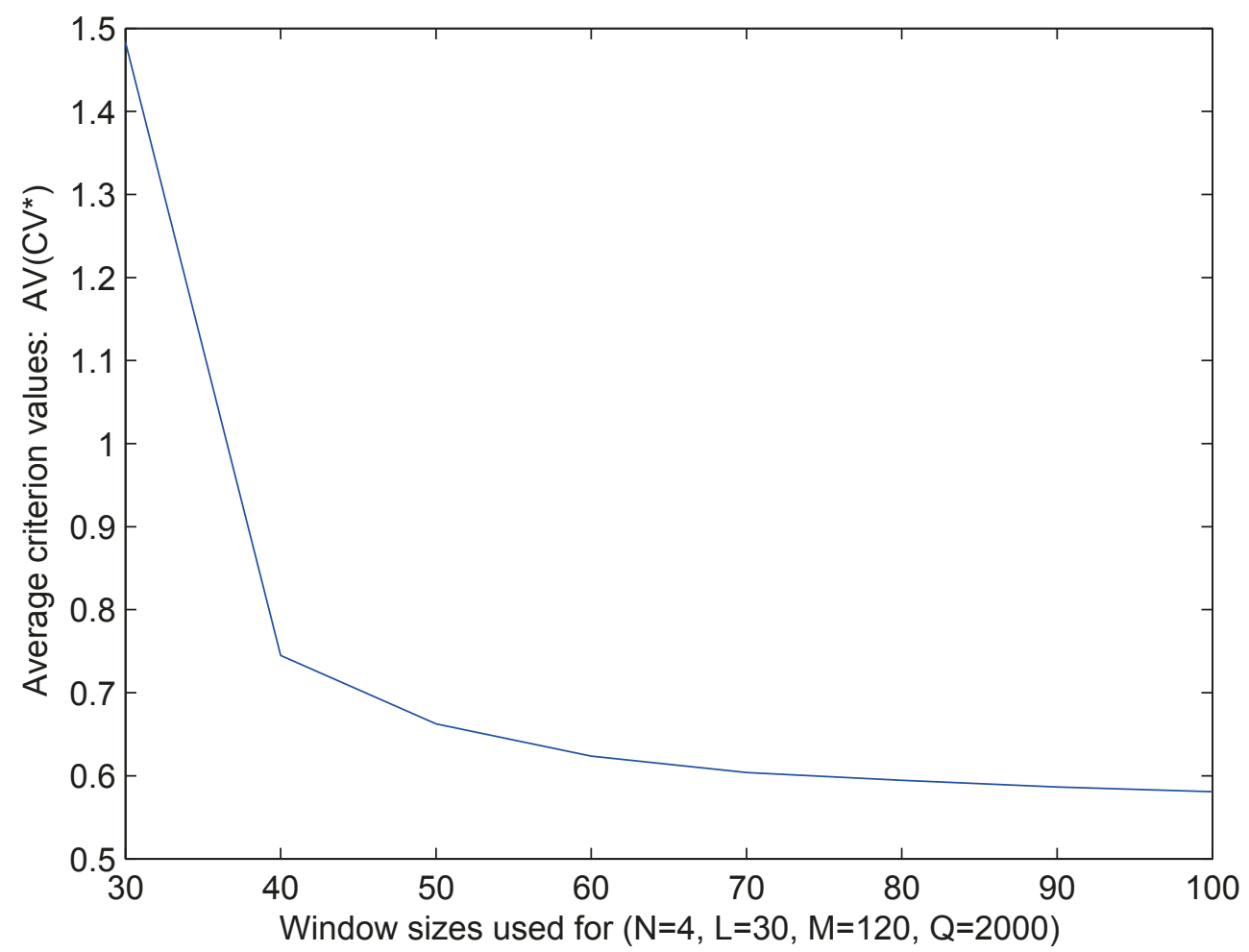

Figure 5.21: Average selected criterion values $\mathrm{AV}\left(\mathrm{CV}^{*}\right)$ vs different window sizes.

It can be seen from Figure 5.21 that the average criterion value is decreasing with the increasing of windows sizes. After certain value (e.g., 70), the average criterion value almost stays flat.

To further show the distribution of selected criterion values (represented as "CV*") with different window sizes, we use the same configuration as Table 5.2 to simulate the selected criterion values at different window sizes and draw the histogram of the results in the following figures. 


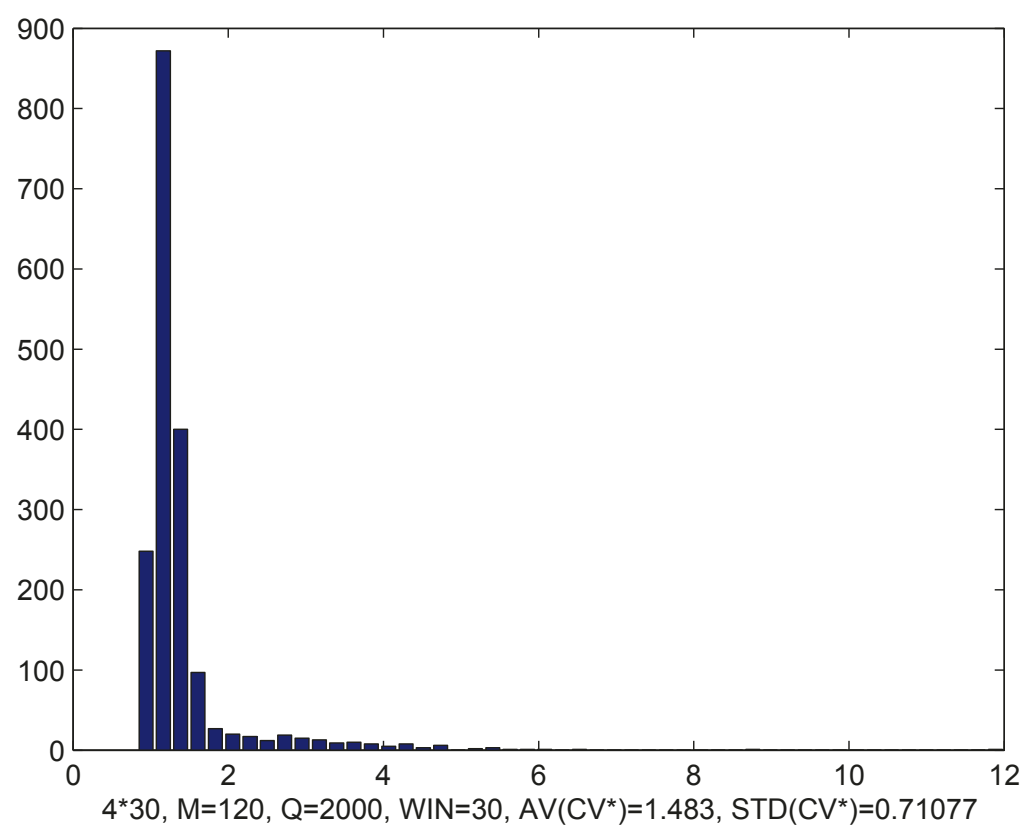

Figure 5.22: Histogram of the selected criterion values $\mathrm{CV}^{*}, \mathrm{WIN}=30$.

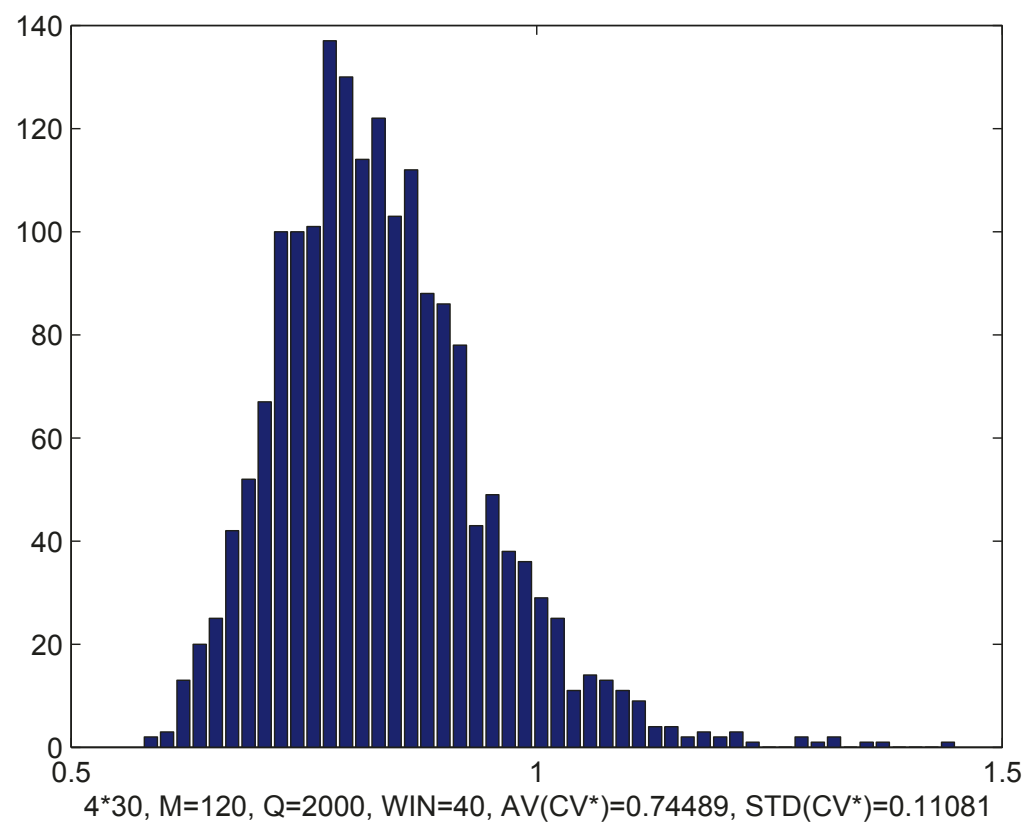

Figure 5.23: Histogram of the selected criterion values $\mathrm{CV}^{*}, \mathrm{WIN}=40$. 


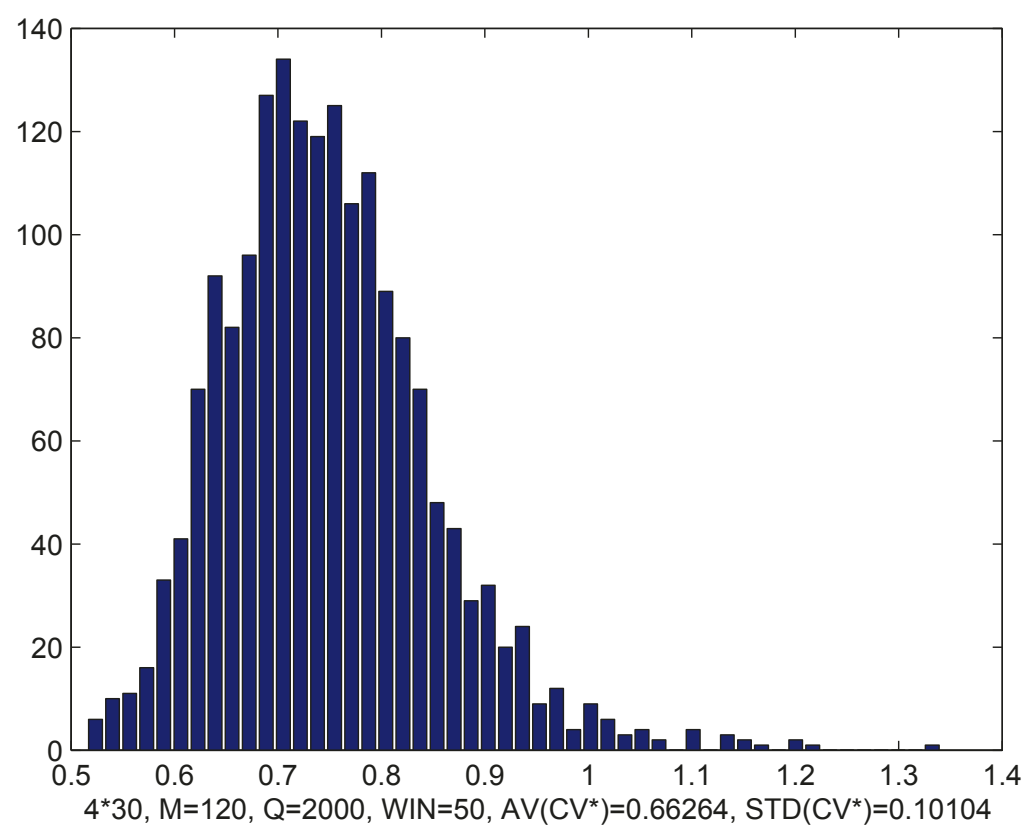

Figure 5.24: Histogram of the selected criterion values $\mathrm{CV}^{*}, \mathrm{WIN}=50$.

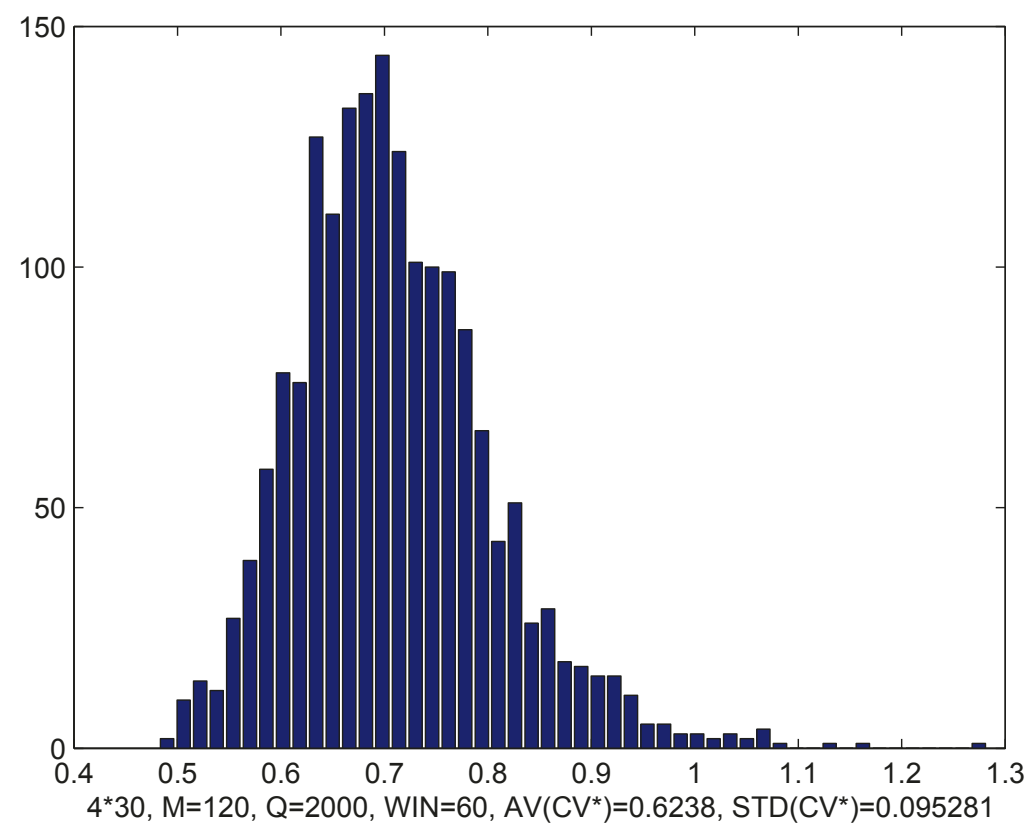

Figure 5.25: Histogram of the selected criterion values $\mathrm{CV}^{*}, \mathrm{WIN}=60$. 


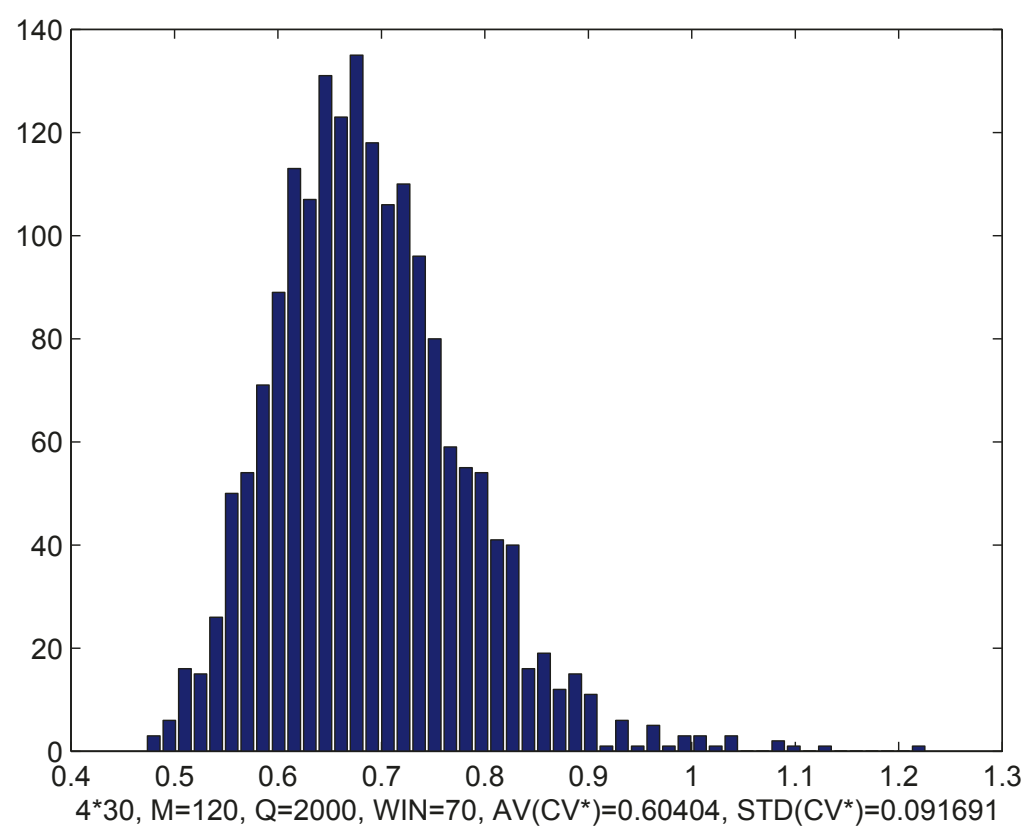

Figure 5.26: Histogram of the selected criterion values $\mathrm{CV}^{*}, \mathrm{WIN}=70$.

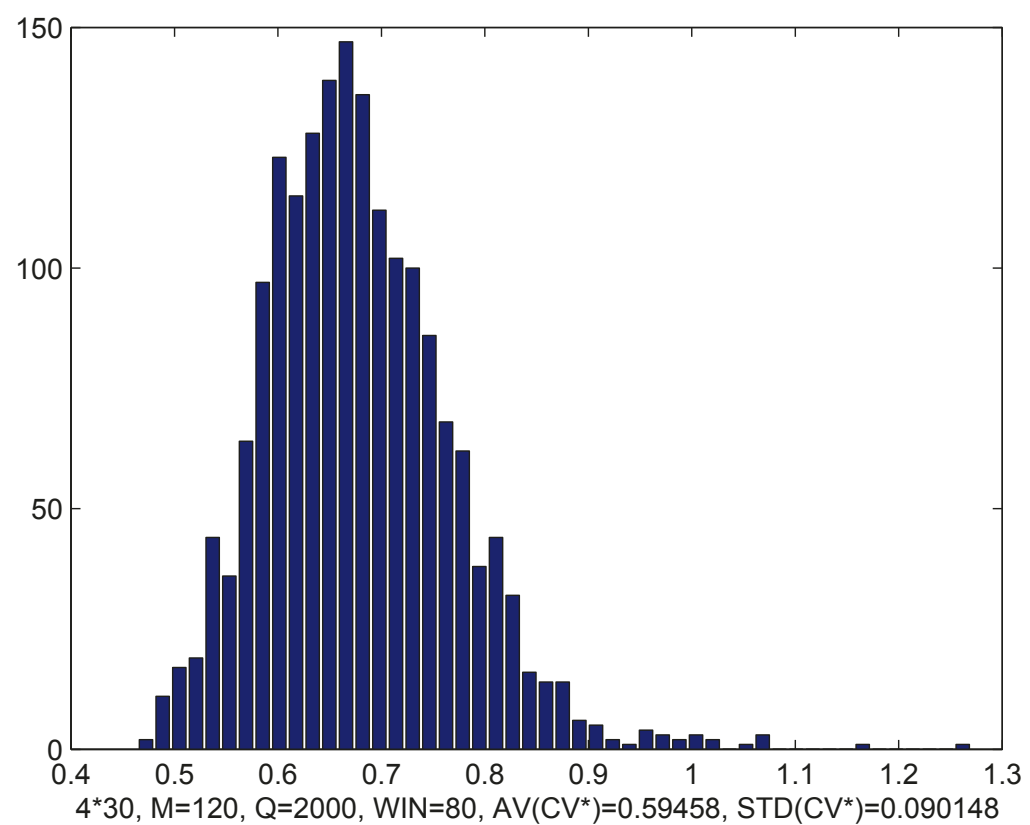

Figure 5.27: Histogram of the selected criterion values $\mathrm{CV}^{*}, \mathrm{WIN}=80$. 


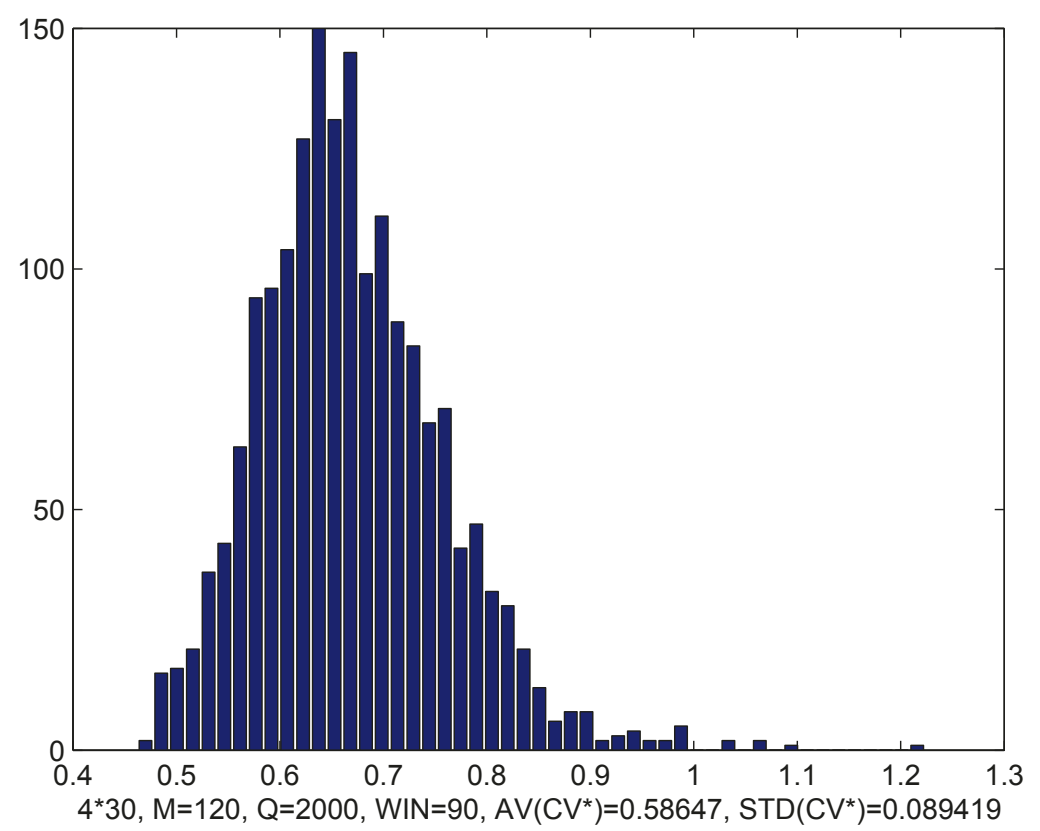

Figure 5.28: Histogram of the selected criterion values $\mathrm{CV}^{*}, \mathrm{WIN}=90$.

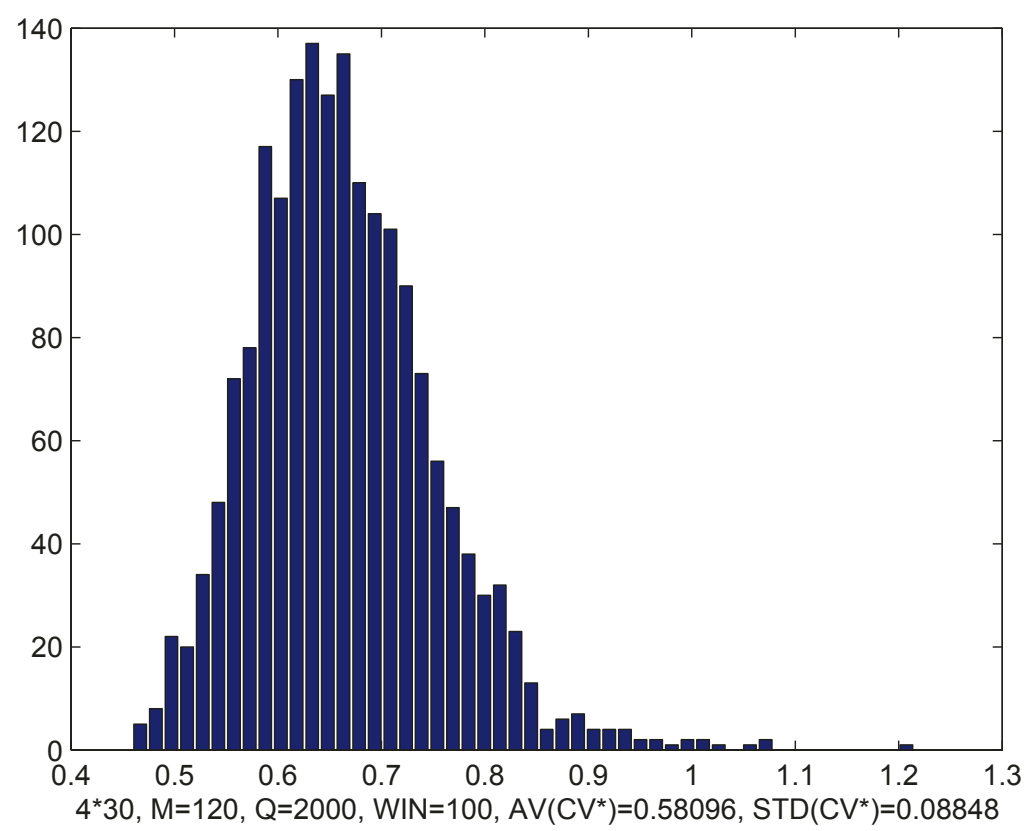

Figure 5.29: Histogram of the selected criterion values $\mathrm{CV}^{*}, \mathrm{WIN}=100$.

From Figures 5.22-5.29, we can see that the distribution of criterion value is 
closely related with the window size. Greater window size leads to denser distribution of criterion values. This can be shown by the average selected criterion values (represented as "AV(CV*)") and standard deviation values in each figure

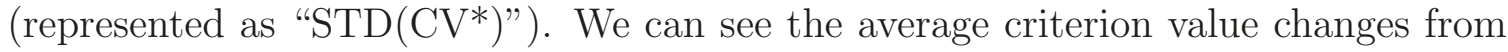
1.483 to 0.58096 and standard deviation changes from 0.71077 to 0.08848 . This can also be shown by Figure 5.30, where CDF curves of selected criterion values for $\mathrm{WIN}=80,90,100$ are almost overlapped. This is compliant with Figure 5.21, and it can be expected that with the increase of window size to a certain extent, the distribution will be almost invariant.

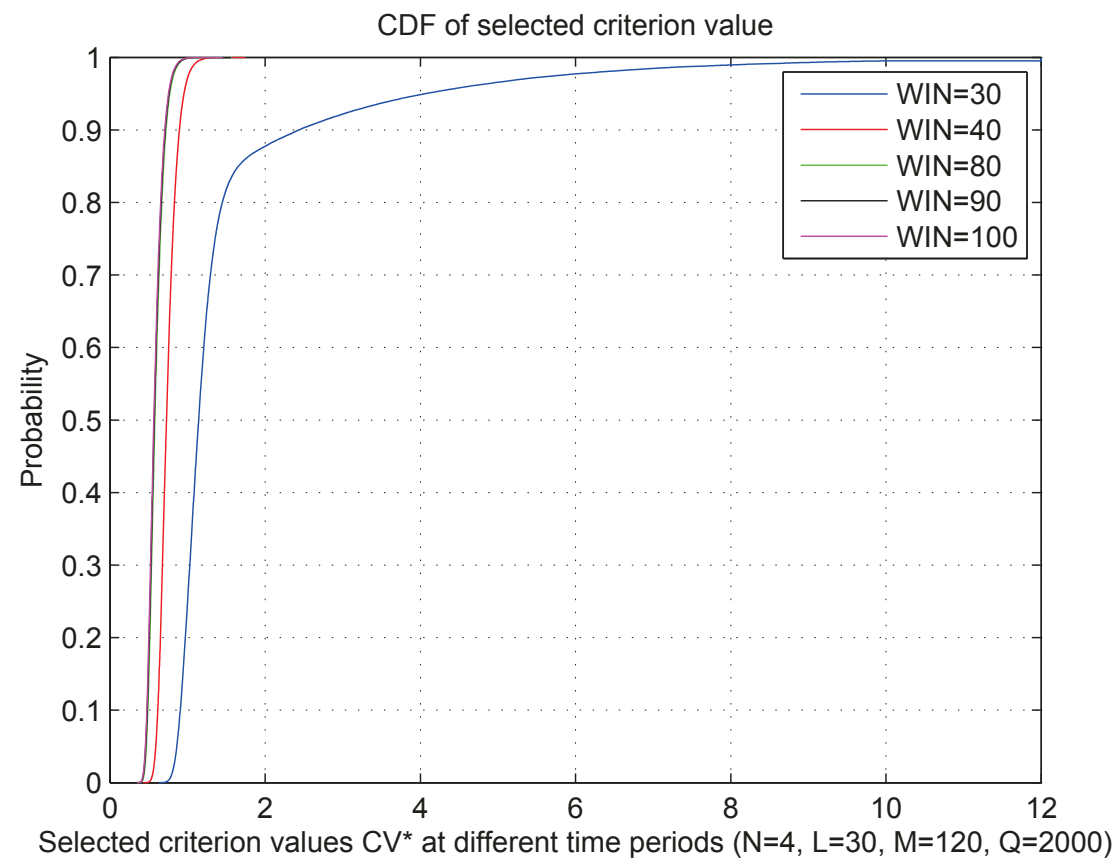

Figure 5.30: $\mathrm{CDF}$ of the selected criterion values $\mathrm{CV}^{*}$.

Next, we will try to find the distribution of number of candidate users at different thresholds (represented as "TH") and window sizes (represented as "WIN"). Here, we use the percentage of average criterion value (represented as " $\mathrm{AV}\left(\mathrm{CV}^{*}\right)$ " for different window sizes WIN) as the threshold, that is , if a user's criterion value is greater than the threshold, it will sent channel reserve request to BSs for arbitration otherwise it 
will keep silent. The simulation results are shown as follows.
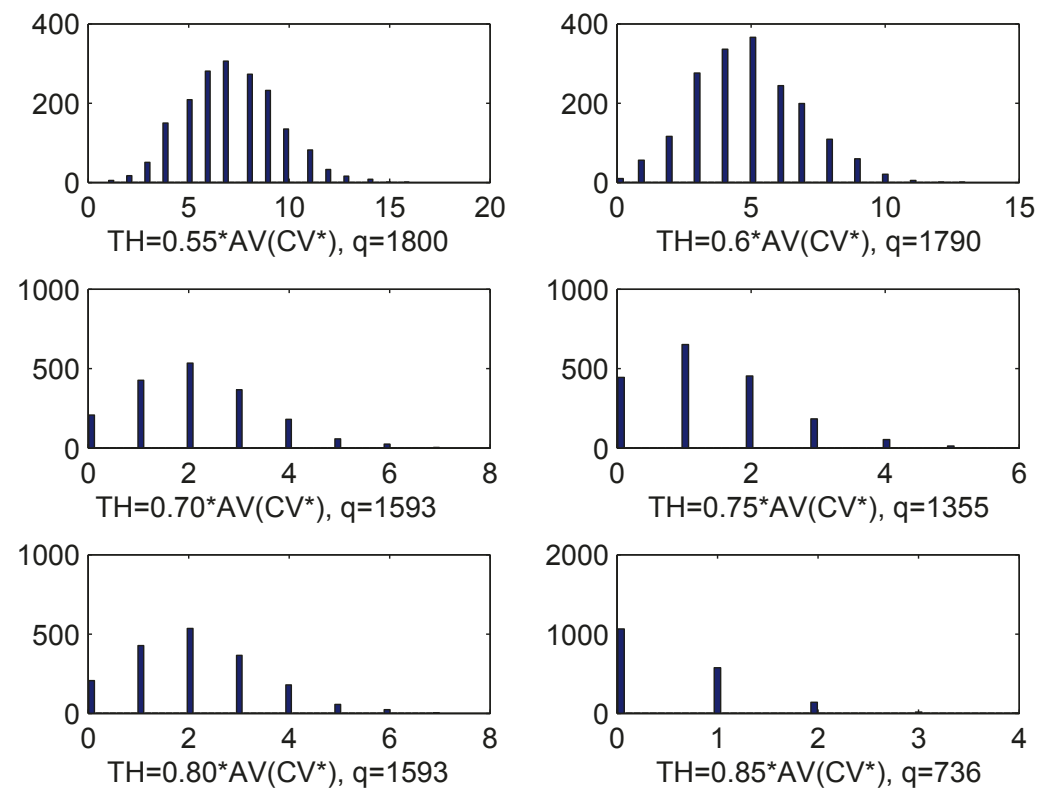

Figure 5.31: Histogram of no. of candidate users at different thresholds (WIN=30).
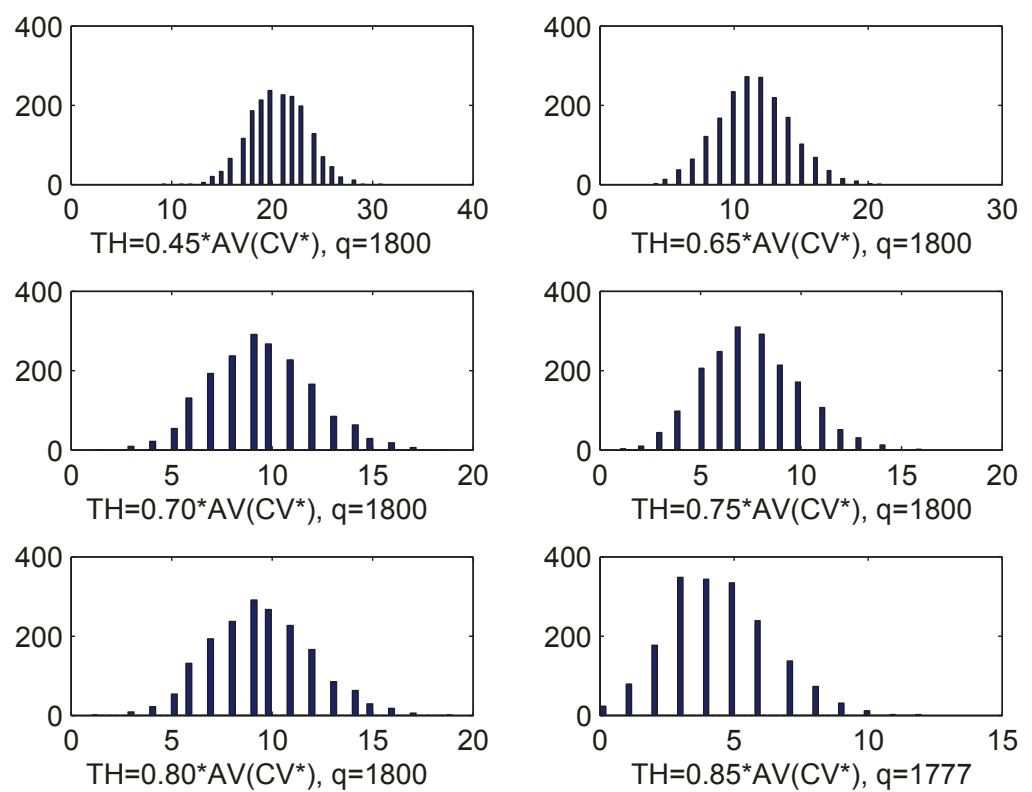

Figure 5.32: Histogram of no. of candidate users at different thresholds (WIN=40). 

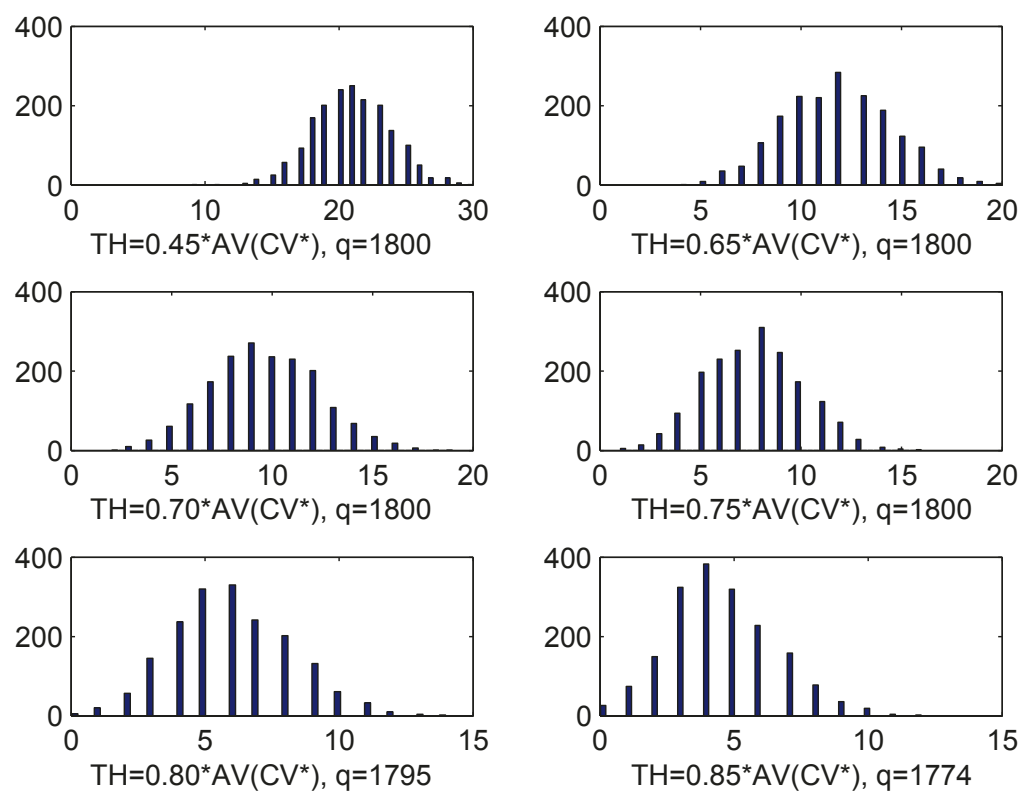

Figure 5.33: Histogram of no. of candidate users at different thresholds (WIN=50).
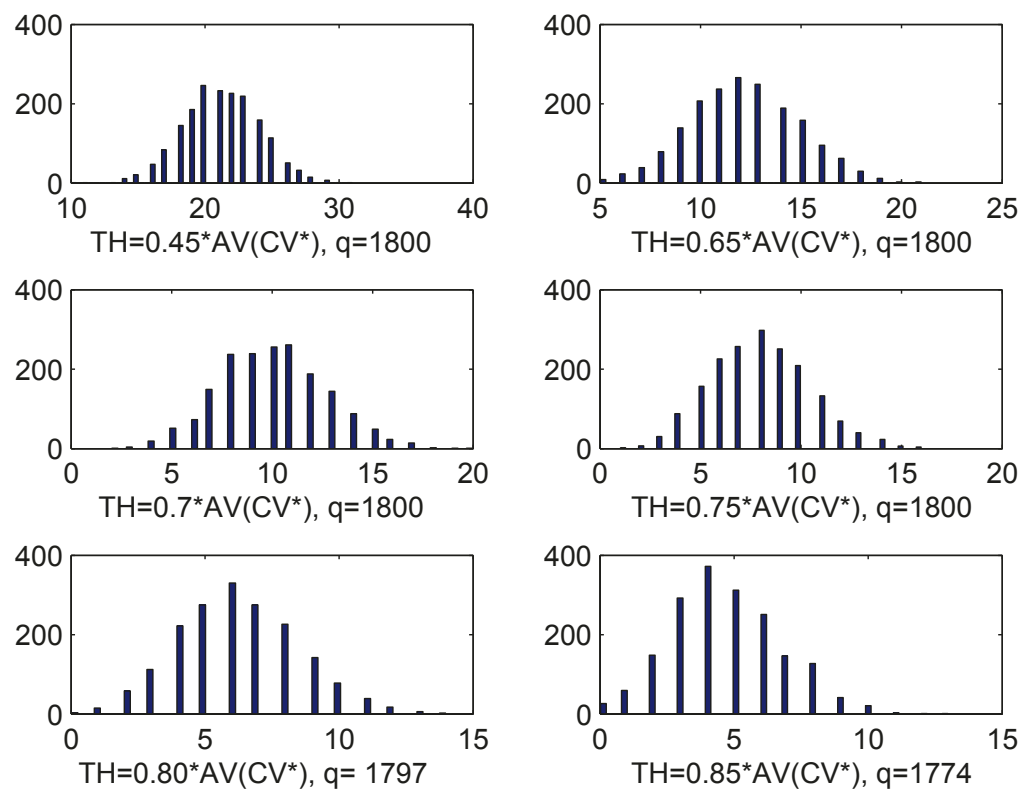

Figure 5.34: Histogram of no. of candidate users at different thresholds (WIN=60). 

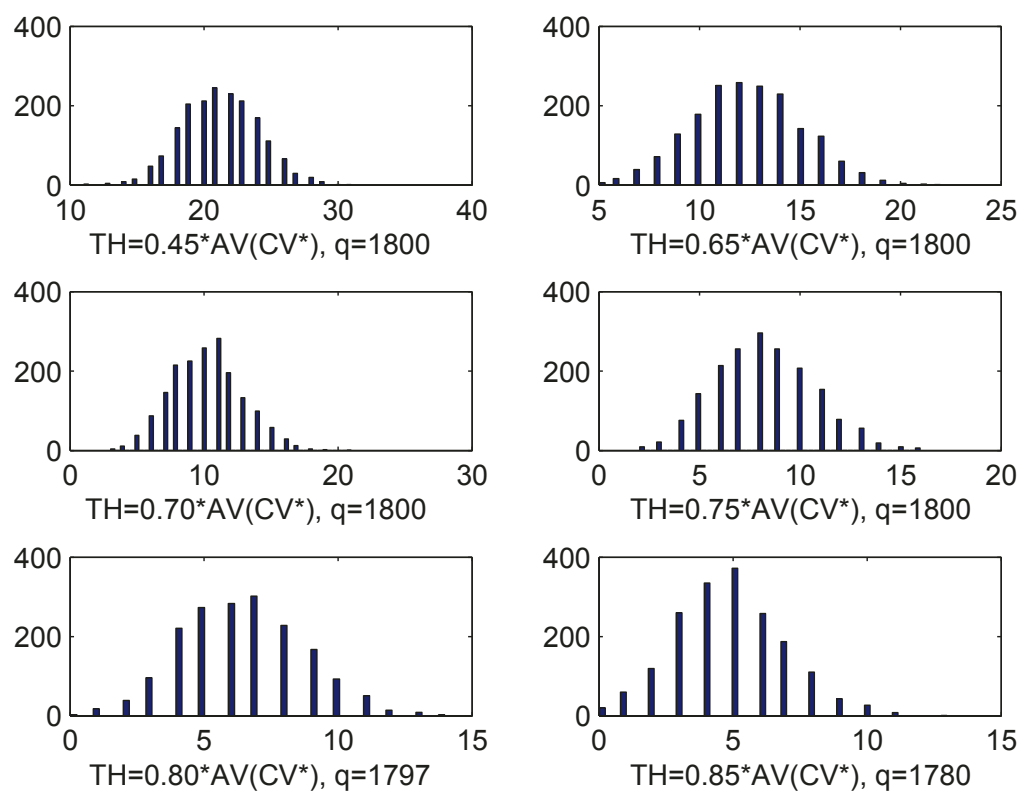

Figure 5.35: Histogram of no. of candidate users at different thresholds (WIN=70).
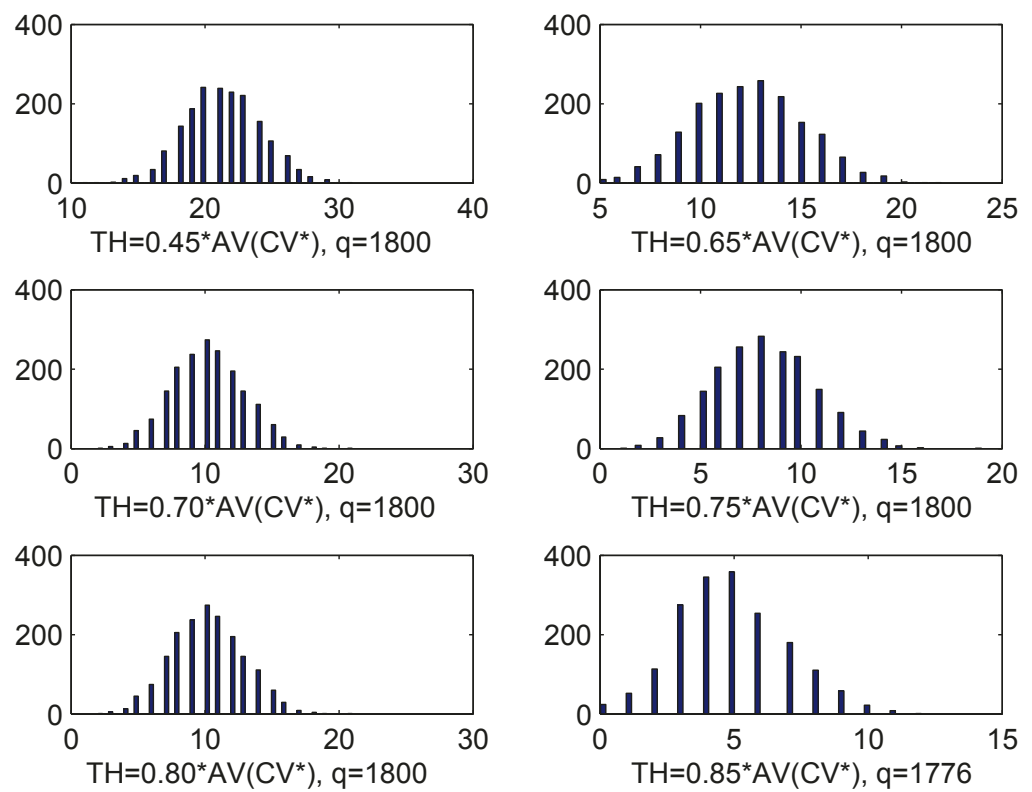

Figure 5.36: Histogram of no. of candidate users at different thresholds (WIN=80). 

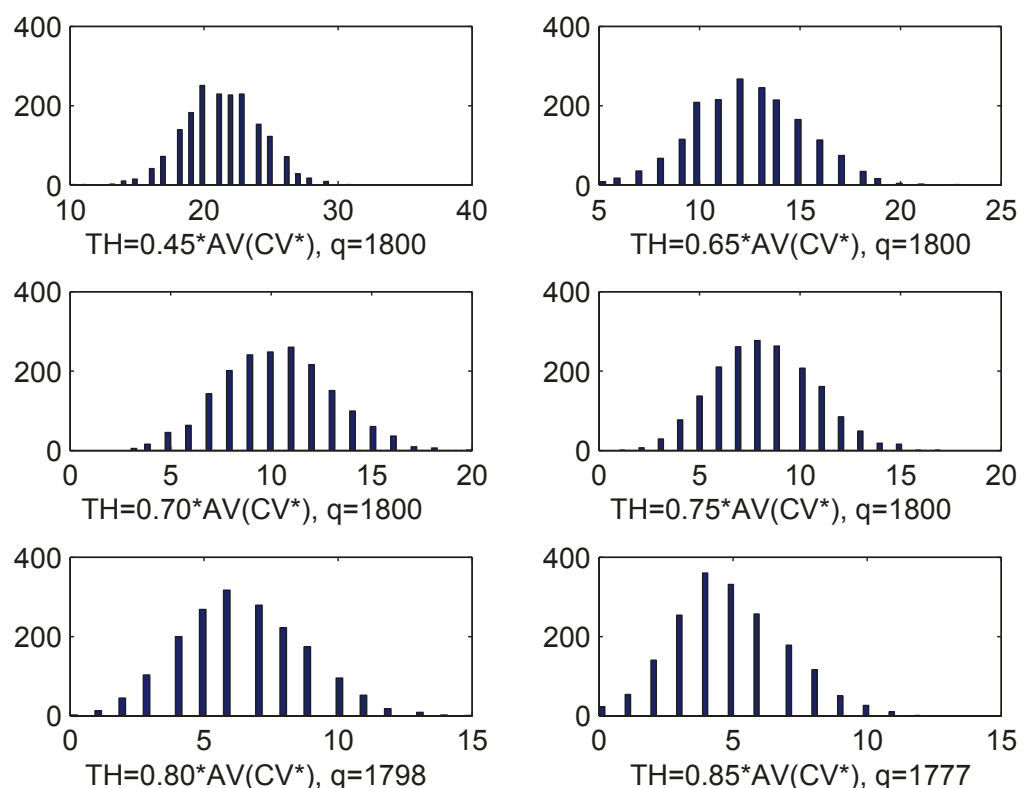

Figure 5.37: Histogram of no. of candidate users at different thresholds (WIN=90).
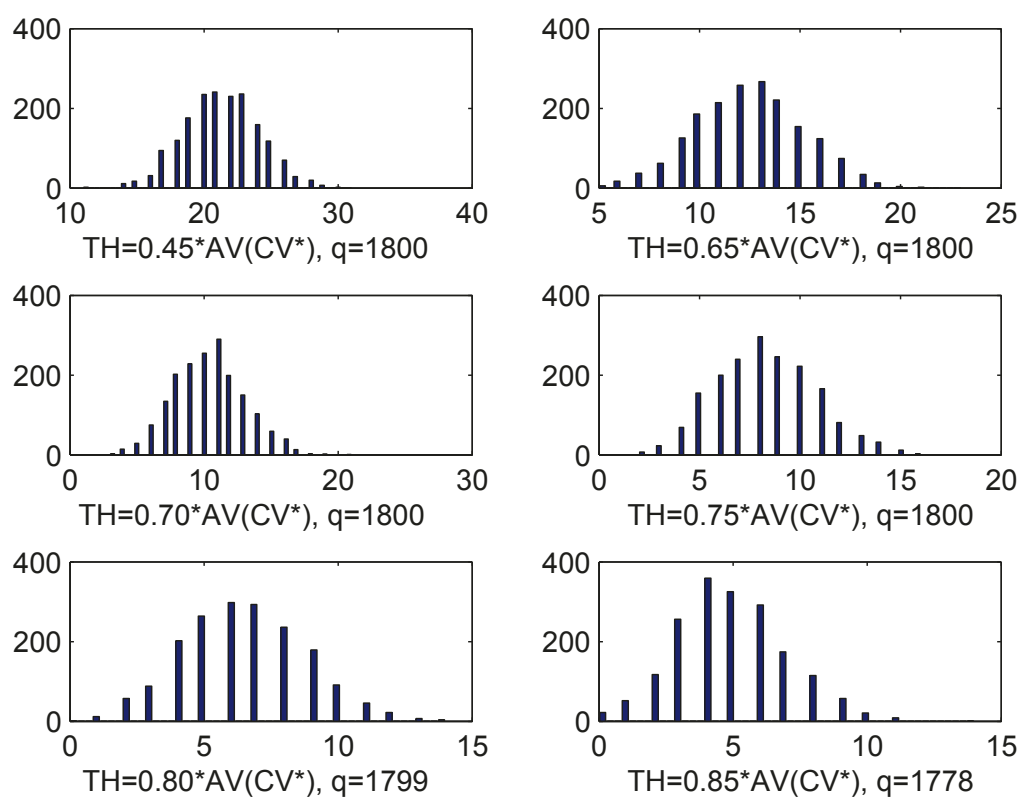

Figure 5.38: Histogram of no. of candidate users at different thresholds (WIN=100).

Here, we analyze the last 1800 time periods of a certain RB (the third RB of 
the second BS), the $\mathrm{x}$-axis represents the number of candidate users, and the $\mathrm{y}$-axis represents the total number of appearance when the number of candidate users equal the value of $\mathrm{x}$-axis. For example, if the value of $\mathrm{x}$-axis is 5 and the value of $\mathrm{y}$-axis is 200, this means that during 1800 time periods, the scenario where the number of candidate users for this RB is 5 happens 200 times. The value of $q$ represents how many times the number of candidate users is at least one. If the number of candidate users for a RB is more than one, this means that this $\mathrm{RB}$ is used by some user and it is not wasted.

From Figure 5.31-5.38, we can see that the suitable thresholds for different window sizes vary. All the suitable thresholds for different window sizes are summarized in Table 5.3.

Table 5.3: Summary of suitable thresholds for different window sizes.

\begin{tabular}{c|c}
\hline \hline Window sizes used in simulation & Suitable thresholds \\
\hline \hline 30 & $(0.55 \sim 0.6) * \mathrm{AV}\left(\mathrm{CV}^{*}\right)$ \\
\hline 40 & $(0.8 \sim 0.85) * \mathrm{AV}\left(\mathrm{CV}^{*}\right)$ \\
\hline 50 & $(0.75 \sim 0.8) * \mathrm{AV}\left(\mathrm{CV}^{*}\right)$ \\
\hline 60 & $(0.8 \sim 0.85) * \mathrm{AV}\left(\mathrm{CV}^{*}\right)$ \\
\hline 70 & $(0.75 \sim 0.8) * \mathrm{AV}\left(\mathrm{CV}^{*}\right)$ \\
\hline 80 & $(0.8 \sim 0.85) * \mathrm{AV}\left(\mathrm{CV}^{*}\right)$ \\
\hline 90 & $(0.75 \sim 0.8) * \mathrm{AV}\left(\mathrm{CV}^{*}\right)$ \\
\hline 100 & $(0.75 \sim 0.8) * \mathrm{AV}\left(\mathrm{CV}^{*}\right)$ \\
\hline
\end{tabular}

For example, the suitable threshold for window size 30 is between 0.55 to 0.60 times of average criterion value. It should be noted that this results are based on the analysis of the third RB of the second BS. For other RBs, the results might be different. This means that in order to find a threshold suitable for all RBs we need to 
analyze the results of all the RBs to get the smallest threshold as the sole threshold for a certain window size. Another key point is that although different window sizes may have different thresholds, the distribution of the number of candidate users for the suitable thresholds is very similar due to centre limit theorem. For example, the dynamic range for the number of candidate users in our simulation is between 0 to 20 and this is true for all RBs. This means: although the thresholds for different window sizes may be very different, the control resources needed for different window sizes do not change dramatically given the same network configuration.

\subsubsection{Cumulative Distribution Function of User Data Rate}

We also calculate the cumulative distribution function of user data rate at different time periods, the results are shown in Figure 5.39. 


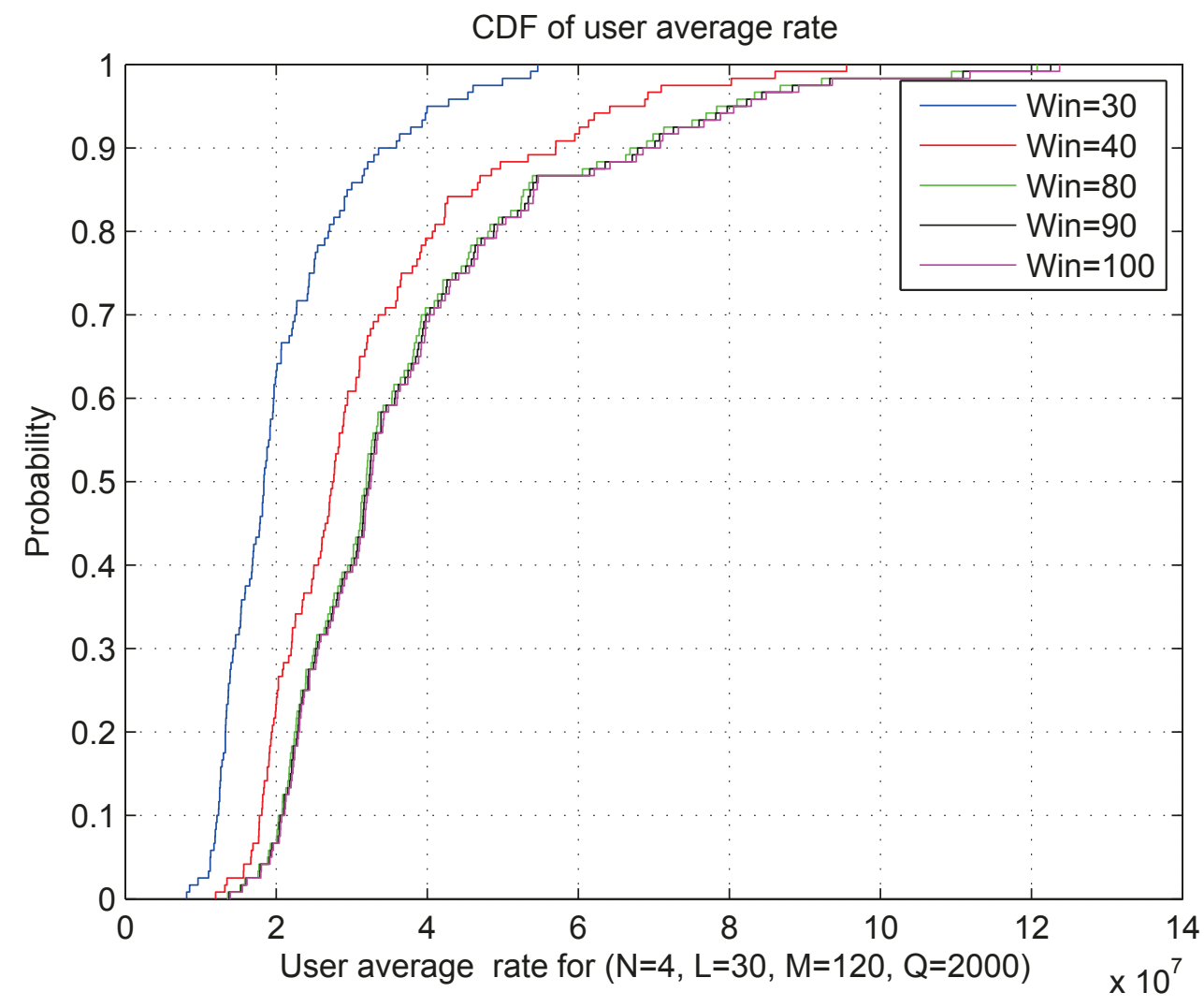

Figure 5.39: CDF of user data rate at different time periods.

From Figure 5.20, we know that given the same number of time periods for simulation, the more the window size, the less the value of Jain's fairness index. This implies that the data rate will be more "even" with smaller window size and the curve will be steeper. Thus the CDF curve should be shift to the right with the increase of window sizes as shown in Figure 5.39. It should also be noted greater window sizes lead to greater user average data rate. This means we need to balance between fairness and throughput. This is similar in Scenario I, where fairness is very much sacrificed to achieve sum throughput maximization. 


\subsubsection{Steps for the Selection of Window Sizes and Thresholds}

Based on the above analysis, the steps for finding window sizes and thresholds are recommended as follows:

1) Try to find the suitable window size used for Algorithm II, the smaller the window size the better fairness and the smaller average data rate we can get. It should also be mentioned that the window size can not be too small otherwise it is very likely that there are quite a number of users whose average rate may be zero due to being unable to get $\mathrm{RBs}$ in such short time periods. Then Algorithm II may not come into effect and the system model is more like a pure Aloha model.

2) Once window size is decided, then try to search for the smallest threshold among all RBs. This smallest threshold can be the candidate threshold used with the decided window size.

\subsubsection{Mass Traffic Handling in Scenario II}

If unexpected mass traffic happens, the performance of Algorithm II will also be severely influenced. First, the window size used for averaging user data rate may be too small to support unexpected large number of users. Like what we have discussed in the above section, the system model will be like a pure Aloha model. The Algorithm II will not be valid. The control resources might also not be enough to support large number of users. If we do not increase the number of RBs, then the feasible window size may be very large and may not be able to get good fairness. To handle this scenario effectively, we need to expand the network configuration to meet the requirements of mass traffic. 


\subsection{Comparisons on the Results of Two Scenarios}

For fairness, we can see from Figure 5.15 and Figure 5.20 that the algorithm used in Scenario II can provide much better fairness than that used in Scenario I.

For spectrum efficiency, it can be seen from Figure 5.2-5.5 and Figure 5.39 the algorithms used in both scenarios can provide high spectral efficiency due to exploiting of multiuser diversity. The differences lie in that the algorithm used in Scenario II achieves this as a long term effect by using a series of time periods while the algorithm used in Scenario I exploits multiuser diversity for every time period to get this result.

For the tools used in algorithms, Scenario I uses two thresholds $\left(H_{t h}\right.$ and $\left.\alpha\right)$ to achieve sum throughput maximization and a low level of control overhead. Scenario II balances between fairness and throughput by adjusting the window size (WIN).

\subsection{Summary}

In this chapter, simulation results for two scenarios are presented.

1) For scenario I (sum throughput maximization), it shows that the peak throughput is directly related to the threshold $H_{t h}$ and by adjusting this value properly, we can achieve good sum throughput performance. It should also noted that this value is related to the number of users. By tuning the attenuation factor $\alpha$, we can effectively control the number of potential channel reservation requests, which mandates the number of control resources.

2) For scenario II (proportional fairness approximation), the simulation results show that our algorithm can achieve good fairness performance as well as throughput performance. By adjusting window size, we can trade off between fairness and throughput. We also provide the method for deciding window sizes and thresholds. 


\section{Chapter 6}

\section{Discussion on Some Implementation}

\section{Issues}

\subsection{Channel Request Collision Avoidance}

In our algorithm framework (Figure 3.2), whether it is for the goal of sum throughput maximization or proportional fairness approximation in uplink data transfer, we need to address channel request collision problem at BS. Fortunately, we have the feasible solutions to control the number of channel reservation requests in both the scenarios of sum throughput maximization and proportional fairness approximation. Considering this, what we need to handle is channel request collision avoidance with just limited number of channel reservation requests. 


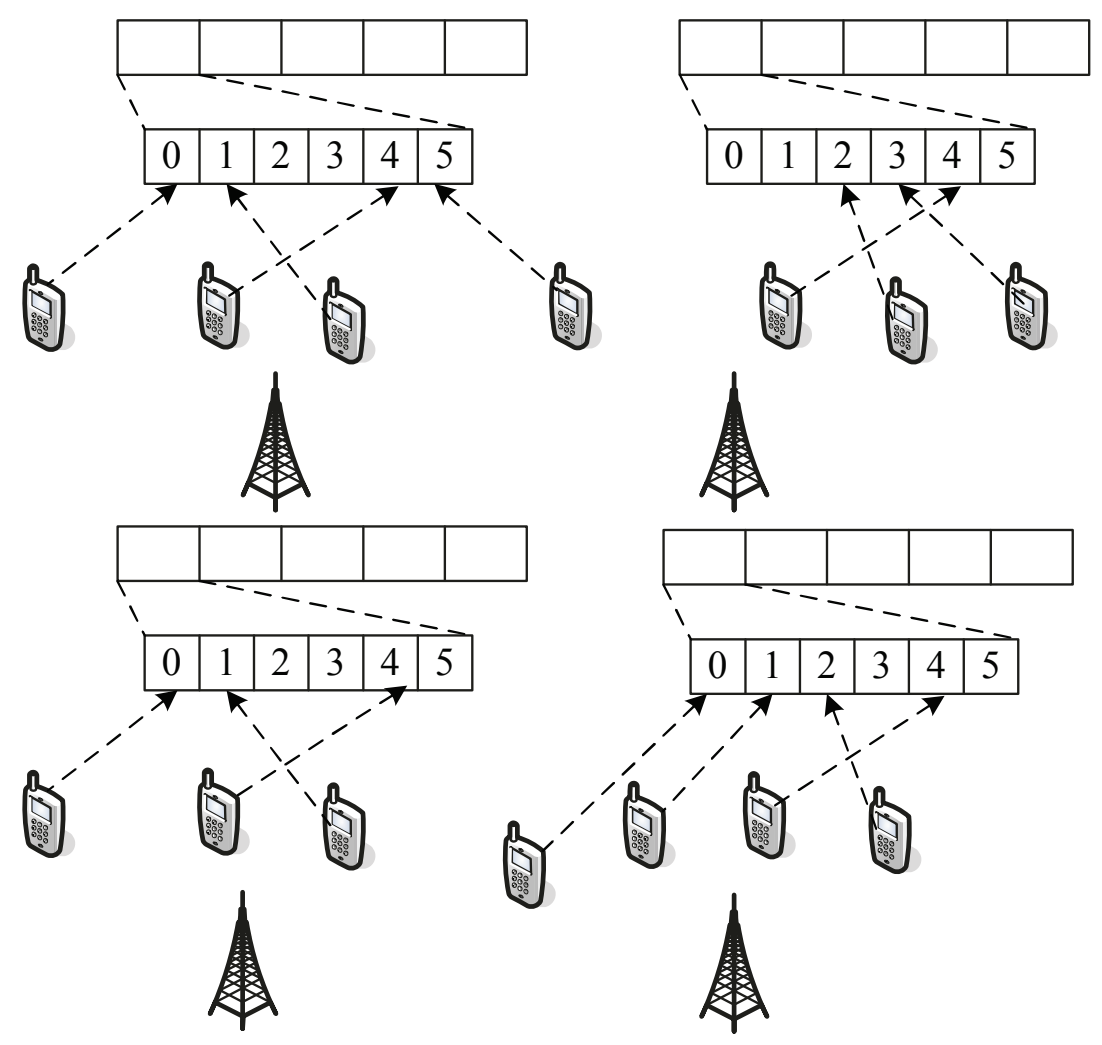

Figure 6.1: Channel request collision avoidance.

To avoid channel request collision effectively, we propose the following method to resolve this problem:

1) Control channels for receiving requests can be divided into several mini-slots with different indexes.

2) Each candidate user uses a common Hash function to calculate its mini-slot index, then send request using this mini-slot.

3) Ideally, all the requests will be distributed in different mini-slots, BSs just need to decode the requests of different mini-slots. In case of collisions in one mini-slot, BSs just tries to decode the requests and ignores it if impossible to decode. 
The above method can be illustrated by Figure 6.1, where every BS has its control channels divided into several mini-slots (for example, 5 mini-slots ). Using a Hash function, every candidate user will be assigned different index. The probability of collision depends on the performance of Hash function. If designed properly, chances are that they will not collide. BSs will decode all these requests slot by slot and select the user with largest criterion value and broadcast the arbitration results.

\subsection{Downlink Data Transfer Discussion}

Although our research in this thesis focuses on uplink data transfer, the ideas for uplink data transfer can be used for downlink data transfer as well. Based on the channel reserve requests, BSs can select the "best" user for downlink data transfer. To some extent, BSs can use uplink arbitration to serve downlink data transfer. This idea is applicable for both TDD system and FDD system.

1) For a TDD system, the result of channel estimation is applicable to both downlink and uplink data transfer. It is BSs' own choice on whether to use the result of channel estimation for downlink or uplink data transfer.

2) For a FDD system, the result of downlink channel estimation is applicable to downlink data transfer. Terminals can sent channel reserve request to BSs for downlink channel arbitration. In this way, BSs do not need to collect all users' channel information and just need to select from candidate users, which will greatly reduce the number of control resources. 


\subsection{Pilot Signal and System Information Broad- cast}

To facilitate the reception of pilot signals and system information, it is suggested that different BSs should use different time slots for broadcast since our system is a multichannel system. If the pilot signals and system information from different base stations are sent in different time slots, terminals can easily decode the signals in a frame, this will simplify the design of terminals without compromising performance.

\subsection{Dynamic Network Adaption}

It can be deduced that our algorithms are closely related with the network conditions such as the number of users, distribution of users, the number of control resources, operators' policies, etc. It is the duty of BSs to adaptively broadcast the needed parameters of algorithms to meet all these requirements. BSs can get all these information from OAM (Operation and Management) or core network nodes.

\subsection{Quantization of Criterion Values}

In practice, we do not need to send the exact criterion values. What we need is to quantize the value into many ranges represented by several bits which can be sent with channel reserve requests. 


\section{Chapter 7}

\section{Conclusions and Future Research}

\section{Directions}

\subsection{Conclusions}

This thesis investigates distributed uplink random access method in multichannel and multicell scenario. In our proposed algorithm framework, terminals play a big part in channel estimation, calculating criterion values and sending channel reserve requests for BSs for arbitration. BSs arbitrate received channel reserve requests and broadcast the specific parameters of algorithms.

Multiuser diversity can be used for achieving high sum throughput. This is very prominent when there are a large number of users contending for channels. Our simulation results show that by exploiting multiuser diversity we can achieve sum throughput comparable to the results of centralized scheduling without causing too much load at BS.

Multipath fading is also a factor that can be fully exploited. Due to fading, it would be rare for a terminal to have more than one good links which means that it will cause considerable interference to other users. Chances are that they have a relatively good link and some bad links at the same time. We can just try to 
sift users with good links and lower interferences to increase spectral efficiency. Our simulation results show that by using different thresholds for communication links and interference links we can take full advantage of multipath fading.

For sum throughput maximization, our simulation shows that the thresholds for communication link can directly decide the peak values of sum throughput. In other word, the thresholds for communication link have optimal values for achieving the peak values of sum throughput. This thresholds are related to the number of users. To achieve the peak values, BSs need to broadcast carefully selected threshold for communication links. The thresholds for controlling interference links does not have much influence on the peak values of sum throughput, but they are very important for controlling the number of candidate users.

Our simulation results show that the sum throughput of multichannel and multicell networks is sensitive to the change of power when the power allocated to terminals is very small. In this range, a small increase of power level can increase the sum throughput dramatically. When the power is increased to a certain level, increasing power level can not boost the sum throughput very much. In conclusion, at low power level, multichannel and multi-cell networks are power-limited while at high power level they are more like interference-limited.

For proportional fairness approximation, our simulation results show that our proposed algorithm can achieve a moderate fairness. The average criterion value changes dramatically when the window size is relatively small and remains steady after the window size increases to a certain range. We can use the average criterion value as a benchmark for setting thresholds to control the number of control resources.

To make channel request collision resolution effectively, we propose that control channels can be divided into mini-slots. Every candidate user's channel reserve requests can be hashed to different mini-slots. This will make BS decode these requests easily and efficiently. 


\subsection{Future Research Directions}

\subsubsection{Power Allocation}

In Chapter 5, we can see that multichannel and multi-cell networks are interference-limited systems at high power level and power-limited systems at low power level. For a real multi-cell system, users may be unevenly distributed and mobile operators may also have different service policies in different locations, how users allocate power under the guidance of BSs to cause as little interference as possible while meeting the basic requirements of users may have practical significance.

\subsubsection{Load Balancing}

In our model and theoretical analysis, we assume that all users are evenly distributed. But this may not be the case in reality. Some BSs may be too heavily used while others may be less used and result in waste of resources. In the area served by highly loaded BSs, some users may not have opportunities for transmission. How to divert these users to less loaded BSs needs consideration.

\subsubsection{QoS Provisioning}

In this thesis research, all users are treated equally by the algorithms. But in practice, at service level, this kind of service may be lowed in priorities compared to other services such as voice service. On the other hand, at user level, different users may also have different service classes which may be decided by the services used or the tariff applied. Basic ideas are that BSs need to handle channel reservation requests with the consideration of QoS requirements. How to handle these kind of priorities while maintaining fairness is a problem worthy of study. 


\section{List of References}

[1] H. Wu and Y. Pan, Eds., Medium Access Control In Wireless Networks. Nova Science Publishers, Inc., 2008.

[2] A. S. Tanenbaum, Computer Networks, 4th. Upper Saddle River : Prentice Hall PTR, 2003.

[3] C. E. Spurgeon, Ethernet: The Definitive Guide. Nutshell Handbook. O'Reilly, 2000 .

[4] A. P. N. Prasad, WLAN Systems \& Wireless IP for Next Generation Communications. Artech House, 2002.

[5] L. Garcia, Communication Networks. The McGraw Hill Companies, 2000.

[6] T. Rappaport, Wireless Communications: Principles and Practice, 2nd Edition. Prentice Hall, 2002.

[7] A. Goldsmith, Wireless Communications. Cambridge University Press, 2005.

[8] O. Tickoo and B. Sikdar, "Queueing analysis and delay mitigation in IEEE 802.11 random access MAC based wireless networks," in INFOCOM 2004. Twenty-third AnnualJoint Conference of the IEEE Computer and Communications Societies, 2004.

[9] Y. S. Han, J. Deng, and Z. Haas, "Analyzing multi-channel medium access control schemes with ALOHA reservation," IEEE Transactions on Wireless Communications, vol. 5, p. 8, 2006.

[10] A. Kaur and M. Gregory, "Performance analysis of random multiple access protocols used in wireless communication," in 6th International Conference on Broadband and Biomedical Communications (IB2Com), 2011. 
[11] Z. J. Haas and J. Deng, "Dual busy tone multiple access (DBTMA): A multiple access control scheme for ad hoc networks." IEEE Transactions on Communications, vol. 50, pp. 975-985, 2002.

[12] N. Jain, S. Das, and A. Nasipuri, "A multichannel CSMA MAC protocol with receiver-based channel selection for multihop wireless networks," in Proceedings of IEEE 9th International Conference on Computer Communications and Networks (ICCCN'01), 2001.

[13] M. A. Marsan and D. Foffinella, "Multichannel local area network protocols," IEEE Journal on Selected Areas in Communications, vol. SAC-1, pp. 885-891, 1983.

[14] J. Sartthong and S. Sittichivapak, "Backoff algorithm optimization for IEEE802.11 wireless local area networks," in 9th International Conference on Electrical Engineering/Electronics, Computer, Telecommunications and Information Technology (ECTI-CON), 2012.

[15] A. C. V. Gummalla and J. O. Limb, "Wireless medium access control protocols," IEEE Communications Surveys \& Tutorials, vol. 3, pp. 2-15, 2000.

[16] R. L. N. Qiang and T. Thierry, "A survey of QoS enhancements for IEEE 802.11 wireless LAN," Journal of Wireless Communications and Mobile Computing, vol. 4, pp. 547-566, 2004.

[17] H. Kwon, H. Seo, S. Kim, and B. G. Lee, "Generalized CSMA/CA for OFDMA systems: Protocol design, throughput analysis, and implementation issues," IEEE Transactions on Wireless Communications, vol. 8, no. 8, pp. 4176-4187, 2009 .

[18] Y.-J. Choi, S. Park, and S. Bahk, "Multichannel random access in OFDMA wireless networks," IEEE Journal on Selected Areas in Communications, vol. 24, no. 3, pp. 603-613, 2006.

[19] R. Knopp and P. Humblet, "Information capacity and power control in single-cell multiuser communications," in IEEE International Conference on Communications, vol. 1, 1995, pp. 331-335.

[20] P. Bender, P. Black, M. Grob, R. Padovani, N. Sindhushyana, and A. Viterbi, "CDMA/HDR: A bandwidth efficient high speed wireless data service for nomadic users," IEEE Communications Magazine, vol. 38, no. 7, pp. 70-77, 2000. 
[21] X. Qin and R. Berry, "Exploiting multiuser diversity for medium access control in wireless networks," in INFOCOM 2003, Twenty-Second Annual Joint Conference of the IEEE Computer and Communications., vol. 2, 2003, pp. 1084-1094.

[22] G. Miao, Y. Li, and A. Swami, "Channel-aware distributed medium access control," IEEE/ACM Transactions on Networking, vol. 20, no. 4, pp. 1290-1303, 2012.

[23] Y. Yu and G. Giannakis, "Opportunistic medium access for wireless networking adapted to decentralized CSI," IEEE Transactions on Wireless Communications, vol. 5, no. 6, pp. 1445-1455, 2006.

[24] G. Ganesan, G. Song, and Y. Li, "Asymptotic throughput analysis of distributed multichannel random access schemes," in IEEE ICC '05, vol. 5, 2005, pp. 36373641.

[25] G. Ganesan, Y. Li, and A. Swami, "Channel aware aloha with imperfect CSI," in IEEE GLOBECOM '06, 2006, pp. 1-5.

[26] K. Bai and J. Zhang, "Opportunistic multichannel aloha: Distributed multiaccess control scheme for OFDM wireless networks," IEEE Transactions on Vehicular Technology, vol. 55, no. 3, pp. 848-855, 2006.

[27] G. Miao, G. Li, and A. Swami, "Decentralized optimization for multichannel random access," IEEE Transactions on Communications, vol. 57, no. 10, pp. 3012-3023, 2009.

[28] W.-Y. Shin, D. Park, and B. C. Jung, "Can one achieve multiuser diversity in uplink multi-cell networks?" IEEE Transactions on Communications, vol. 60, no. 12, pp. 3535-3540, 2012.

[29] X. Qin and R. Berry, "Opportunistic splitting algorithms for wireless networks with fairness constraints," in 4th International Symposium on Modeling and Optimization in Mobile, Ad Hoc and Wireless Networks, 2006, pp. 1-8.

[30] P. Viswanath, D. Tse, and R. Laroia, "Opportunistic beamforming using dumb antennas," IEEE Transactions on Information Theory, vol. 48, pp. 1277-1294, 2002 .

[31] F. P. Kelly, A. K. Maulloo, and D. K. Tan, "Rate control for communication networks: shadow prices, proportional fairness and stability," Journal of the Operational Research society, vol. 49, pp. 237-252, 1998. 
[32] R. Jain, D.-M. Chiu, and W. R. Hawe, A quantitative measure of fairness and discrimination for resource allocation in shared computer system. Eastern Research Laboratory, Digital Equipment Corporation, 1984.

[33] H.-C. Yang and M.-S. Alouini, Order Statistics in Wireless Communications: Diversity, Adaptation, and Scheduling in MIMO and OFDM systems. Cambridge University Press, 2011.

[34] 3GPP TR 25.996 V11.0.0, Spatial Channel Model for Multiple Input Multiple Output (MIMO) simulations (Release 11), 2012-09. 